\title{
Numerical Modeling of Silicon Photodiodes for High-Accuracy Applications with PC-1D
}

\section{NISTIR 4592}

J. Goist

D. Chandler-Horowitz

U.S. DEPARTMENT OF COMMERCE National Institute of Standards and Technolosy

Semlconductor Electronics Division Electronics and Eloctrical Engineering Laboratory Calthorburg, MD 20899

\section{A. M. Robinson \\ C. R. James \\ Universtty of Aberta \\ Aberta, Canada T6G $2 G 7$}

\section{R. Kohler \\ R. Goebel}

BIPM

F-92310, Sovre, France 



\section{Numerical Modeling of Silicon Photodiodes for High-Accuracy Applications with PC-1D}

\section{J. Geist \\ D. Chandler-Horowitz}

U.S. DEPARTMENT OF COMMERCE Natjonal Institute of Standards and Technotogy Semiconductor Electronics Division Eloctronics and Eloctrical Englnoering Laboratory Galtheraburg, MD 20899
A. M. Robinson
C. R. James

Universtty of Nborta

Alborta, Canada T6Q 207

\section{R. Kohler \\ R. Goebel}

BIPM

F-92310, Sovre, Franco

Jenuary 1992

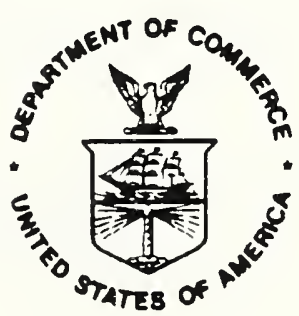

U.S. DEPARTMENT OF COMMERCE Robert A. Mosbachor, Socretary MATHONAL WSTITUTE OF STANDARDS AND TECHNOLOCY

John W. Lyone, Director 

NLWERICAL MODELING OF SILICON PHOTODIODES

FOR HIGH-ACCURACY APPLICATIONS WITH PC-1D

Table of Contents

Introduction to NISTIR 4592

Page

References

Part I: Simulation Programs

Abstract

1. Introduction . . . . . . . . . . . . . . . . . . . 2

2. Description of PC-1D . . . . . . . . . . . . . . . . . 3

2.1 Opening Menu . . . . . . . . . . . . . . . . . 3

2.1.1 Materials Properties . . . . . . . . . . . . . . . . . . . . . . . 5

2.1.2 Device Properties . . . . . . . . . . . . . . . . . . . . . . . . 7

3. The Photodiode Modeling Shell Programs . . . . . . . . . . . . . . . 10

3.1 Operation of RUN PC1D.BAT . . . . . . . . . . . . . . . . . . . . 10

3.2 Features of MAKE_PRM and READ_PDF . . . . . . . . . . . . . . . 12

4. Conclusion . . . . . . . . . . . . . . . . . . 15

5. References . . . . . . . . . . . . . . . . . . . 15

Appendix I - A Listing of MAKE_PRM.PAS That Compiles under Turbo

Pascal Version 5.5 . . . . . . . . . . . . . . . 16

Appendix II - A Listing of READ_PDF.PAS That Compiles under Turbo Pàscal Version 5.5 . . . . . . . . . . . . . . . . . . . . . 29

Appendix III - A Sample of the Screen Produced by MAKE PRM.EXE When RUN PC1D.BAT is Executed . . . . . . . . . . 33

Part II: Interpreting Oxide-Bias Experiments . . . . . . . . . . . . . . 34

Abstract . . . . . . . . . . . . . . . . . . . . . . . . 34

1. Introduction . . . . . . . . . . . . . . . . . . 34

2. EG\&G UV444B Photodiode . . . . . . . . . . . . . . . . . . . . 35

3. Hamamatsu 1337 Photodiode . . . . . . . . . . . . . . . . . . 42

4. UDT UV100 Photodiode . . . . . . . . . . . . . . . . . . . . 47

5. Conclusion . . . . . . . . . . . . . . . . . . . 53

6. References ..................... . . . 55

Part III: Interpolating and Extrapolating Internal Quantum-Efficiency Calibrations . 56

Abstract . . . . . . . . . . . . . . . . . . . 56

1. Introduction . . . . . . . . . . . . . . . . . . . 56

2. Errors Associated with the Quantum-Efficiency Approximations . . . . . . 57

3. Errors Associated with Quantum-Deficiency Calculations . . . . . . . . . 68

4. Comparison with Experiment . . . . . . . . . . . . . . . . . . . . 78

5. Conclusion . . . . . . . . . . . . . . . . . . . 82

6. References . . . . . . . . . . . . . . . . 85 


\section{List of Figures}

Page

Part I

1. The opening menu of PC-1D when run in the interactive mode . . . . . . . 4

2. Ratios of the absorption-coefficient data interpolated from Ref. [5] to those calculated from eq (1) of Ref. [3] that were fitted to the data of Ref. [4] . . . . 6

3. Comparison of experimental and simulated linearity measurements on an EG\&G UV444B photodiode at $950 \mathrm{~nm}$ using a single SRH trap level at mid-gap with electron and hole lifetimes of $74.04 \mu \mathrm{s}$. . . . . . . . . . . . . . . . . .

4. Comparison of experimental and simulated linearity measurements on an EG\&G UV444B photodiode at $950 \mathrm{~nm}$ using a single SRH trap level $0.1796 \mathrm{eV}$ above mid-gap with electron and hole lifetimes of $900 \mathrm{~ms}$ and $700 \mathrm{~ms}$, respectively . . 9

5. Listing for the MSDOS batch file RUN PC1D.BAT that supervises the execution of the programs MAKE PRM.EXE, PC-1D.EXE, and READ_PDF.EXE for high-accuracy photodiode modeling . . . . . . . . . . . . . . . . 11

6. Variation in the total photocurrent calculated by $P C-1 D$ as a function of position in the front region of a Hamamatsu 1337 type photodiode due to the effect of the high front region doping concentration

Part II

1. The front-region boron concentration and the equilibrium hole concentration calculated by PC-1D for a typical EG\&G UV444B type photodiode . . . . . 36

2. Ratio of the photocurrent $I\left(V_{0}\right)$ as a function of oxide-bias voltage $V_{0}$ to the photocurrent $\mathrm{I}(0)$ at zero-bias voltage as measured on an EG\&G UV444B silicon photodiode and as calculated with PC-1D with a front-surface recombination velocity $S=6000 \mathrm{~cm} / \mathrm{s}$ and an oxide trapped charge number density $\mathrm{N}_{s,}=1.942 \times 10^{12} \mathrm{~cm}^{-2}$. . . . . . . . . . . . . . . . . . . . . 37

3. The residuals between the experimental data of figure 2 and the simulated data calculated by PC-1D when $\mathrm{S}=6000 \mathrm{~cm} / \mathrm{s}$ and $\mathrm{N}_{\varrho}=1.942 \times 10^{12} \mathrm{~cm}^{-2}$. . . . 38

4. The residuals between the experimental data of figure 2 and the simulated data calculated by PC-1D when $S=5000 \mathrm{~cm} / \mathrm{s}$ and $\mathrm{N}_{a}=2.011 \times 10^{12} \mathrm{~cm}^{-2}$. . . . 39

5. The residuals between the experimental data of figure 2 and the simulated data calculated by $P C-1 D$ when $S=7000 \mathrm{~cm} / \mathrm{s}$ and $\mathrm{N}_{\text {s, }}=1.883 \times 10^{12} \mathrm{~cm}^{-2}$. . . . 40

6. The relative front-region boron concentration for six typical Hamamatsu 1337 type photodiodes

7. Ratio of the photocurrent $I\left(V_{0}\right)$ as a function of oxide-bias voltage $V_{0}$ to the photocurrent $I(0)$ at zero-bias voltage as measured on a Hamamatsu 1337 silicon photodiode and as calculated with PC-1D for the doping distribution corresponding to the upper solid curve in figure 6 with $S=1.835 \mathrm{~cm} / \mathrm{s}$ and $\mathrm{N}_{\text {s, }}=-3.0 \times 10^{12} \mathrm{~cm}^{-2}$. . . . . . . . . . . . . . . . . . . . . . . . . 4

8. Simulated internal quantum deficiencies for the 1337 type photodiode having the 
oxide-bias data shown in figure 7

9. Ratio of the photocurrent $I\left(V_{0}\right)$ as a function of oxide-bias voltage $V_{0}$ to the photocurrent $\mathrm{I}(0)$ at zero-bias voltage as measured on a typical UDT UV100 silicon photodiode and as calculated with PC-1D for $\mathrm{S}=3.0 \times 10^{5} \mathrm{~cm} / \mathrm{s}$ and $\mathrm{N}_{\mathrm{O}}=1.38 \times 10^{12} \mathrm{~cm}^{-2}$

10. Internal quantum-deficiency spectra calculated by PC-1D for $S=3.0 \times 10^{5}$ $\mathrm{cm} / \mathrm{s}, \mathrm{N}_{s s}=1.38 \times 10^{12} \mathrm{~cm}^{-2}$, and rear-region lifetimes $\tau$ of $81 \mathrm{~ms}, 10 \mu \mathrm{s}$, and $1 \mu \mathrm{s}$

11. Internal quantum-deficiency spectra calculated by PC-1D for a rear-region lifetime $\tau=1 \mu$ s with $\mathrm{S}=3.0 \times 10^{5} \mathrm{~cm} / \mathrm{s}$ and $N_{\theta g}=1.38 \times 10^{12} \mathrm{~cm}^{-2}$, with $\tau=1 \mu \mathrm{s}$ with $\mathrm{S}=6.0 \times 10^{5} \mathrm{~cm} / \mathrm{s}$ and $\mathrm{N}_{\theta \theta}=1.38 \times 10^{12} \mathrm{~cm}^{-2}$, and with $\tau=1 \mu \mathrm{s}$ with $\mathrm{S}=3.0 \times 10^{5} \mathrm{~cm} / \mathrm{s}$ and $\mathrm{N}_{\Delta o}=0.69 \times 10^{12} \mathrm{~cm}^{-2}$

12. Comparison of the internal quantum-deficiency spectra calculated for a UV100 type photodiode for a rear-region lifetime $\tau=1 \mu \mathrm{s}$ with $\mathrm{S}=3.0 \times 10^{5} \mathrm{~cm} / \mathrm{s}$ and $\mathrm{N}_{s s}=1.38 \times 10^{12} \mathrm{~cm}^{-2}$ with the absorption-coefficient data in the SIL_WEAK.ABS file, and with the absorption-coefficient data in the SIL_PHIL.ABS file

13. Simulation of the decrease in internal quantum-deficiency of a UV100 type photodiode with reverse bias at $442 \mathrm{~nm}, 468 \mathrm{~nm}$, and $514 \mathrm{~nm}$

Part III

1. The quantum-deficiency spectra $\delta(\lambda, 0,71264 \mathrm{~cm} / \mathrm{s}, \infty)$ and $\delta(\lambda, 0,0,1 \mathrm{~ms})$ and their sum

2. The quantum-deficiency spectra $\delta\left(\lambda, N_{\varrho}, S, \tau_{r}\right)$ for the conditions listed in table 1

3. The front-region doping distributions $N_{A}(x)$ used to calculate the front-region internal quantum-deficiency curves in figure 2

4. Differences between the internal quantum-deficiency spectra of figure 2 for Cases 1, 3, and 4 when normalized to 0.01 at $440 \mathrm{~nm}$, and that for Case 2 when normalized to 0.01 at $440 \mathrm{~nm}$

5. The default, band-to-band recombination lifetime profile calculated by PC-1D for the doping distribution shown as the solid curve in figure 3

6. The internal quantum-deficiency spectra $\delta\left(\lambda, N_{, o}, S, \tau_{r}\right)$ for $\tau_{r}=1 \mathrm{~ms}$, and $\tau_{r}=10 \mathrm{~ms}$ with $N_{\mathrm{se}}=S=0$

7. Differences between the internal quantum-deficiency spectra of figure 6 when normalized to 0.002 at $860 \mathrm{~nm}$

8. Comparison of $\hat{M}_{0}(x), \hat{n}_{i e}(x), \hat{\mu}_{m}(x)$, and $\exp (-\alpha(400 \mathrm{~nm}) x)$ for a 1337 type photodiode with $N_{, s}, S$, and $\tau_{r}$ set as defined in Case 2 of table 1

. Differences between the internal quantum-deficiency spectrum for a 1337 type photodiode satisfying the conditions defined in table 1 for the absorptioncoefficient data of Ref. [14] and for the absorption-coefficient data of Ref. [12] . 74

10. Differences between the internal quantum-deficiency spectra for a 1337 type 
photodiode with $S=0$ and $\tau_{r}=1 \mathrm{~ms}$ for the absorption-coefficient data of Ref. 11' and for the absorption-coefficient data of Ref. [12] . . . . . . . . T5

11. Sum in quadrature of the curves in figures 4 and $9 . \ldots . . . .66$

12. Sum in quadrature of the curves in figures 7 and 10 . . . . . . . 77

13. Comparison of measured data of Ref. [17] with eq (17) normalized to pass through the measured point at 468.18 and $859.1 \mathrm{~nm}$

\section{List of Tables}

Part II

1. Comparison of simulated oxide bias results with experimental results for a UV444B type where Res. Std. Dev. is the standard deviation of the difference between simulated and experimental results . . . . . . . . . . . . . . 41

Part III

1. Recombination-related parameters used with the doping distributions shown in figure 3 to model the quantum-deficiency spectra of Hamamatsu 1337 type photodiodes . . . . . . . . . . . . . . . . . .6 6

2. Values of the parameters to be used in eq (4) for extrapolating a short wavelength internal quantum-deficiency value to longer wavelengths and in eq (5) for extrapolating a long wavelength internal quantum-deficiency value to shorter wavelengths . . . . . . . . . . . . . . . 69

3. Average values of, and uncertainty estimates for the internal quantum efficiency $\epsilon_{f}$, spectral responsivity $\mathrm{R}$, one minus the reflectance $(1-\rho)$ and nonlinearity correction (1 - NL) reported in Ref. [5] at wavelength $\lambda$ for five multiple-reflection radiometers based on 1337 type photodiodes, and internal quantum-deficiency values $\delta_{\boldsymbol{x}}(\lambda)$ calculated from the reported data . . . . . . . . . . . . . . . 80

4. Oxide-bias data $\gamma_{o}$ reported in Ref. [17] for a single 1337 type photodiode, the average internal quantum-efficiency data $\epsilon_{x}$ reported in Ref. [17] for ten multiple-reflection radiometers based on 1337 type photodiodes, and the quantum deficiencies $\delta_{\boldsymbol{x}}(\lambda)$ calculated from these sets of data . . . . . . . .80

5. Comparison of predictions of eq (17) with measured internal quantum-deficiency data $\delta_{x}(\lambda)$ in table 3 for $\lambda_{f}=441.6 \mathrm{~nm}$ and $\delta_{x}\left(\lambda_{r}\right)=0 \ldots$. . . . . . 81

6. Comparison of predictions of eq (17) with internal quantum-deficiency data $\delta_{x}(\lambda)$ derived from oxide-bias data in table 4 for $\lambda_{f}=468.18 \mathrm{~nm}$ and $\delta_{x}\left(\lambda_{r}\right)=0$

7. Comparison of predictions of eq (17) with measured internal quantum-deficiency data in table 4 for for $\lambda_{f}=468.18 \mathrm{~nm}$ and $\lambda_{r}=859.07 \mathrm{~nm}$. . . . . . . . . 83 


\title{
NUMERICAL MODELING OF SILICON PHOTODIODES FOR HIGH-ACCURACY APPLICATIONS
}

\author{
Introduction to NISTIR 4592
}

The purpose of this National Institute of Standards and Technology Interagency/Internal Report (NISTIR) is to provide the source code for two Turbo Pascal* 5.5 programs and an MSDOS batch program, along with a paper [1] that describes the programs and provides examples of their use. These three programs serve as a batch mode interface to support high-accuracy photodiode modeling with Version 2 of the semiconductor device modeling program PC-1D [2]. These programs are useful because the interactive user interface of PC-1D is optimized for solar cell modeling, and it is somewhat difficult to access the highest accuracy available from PC-1D through this interface.

The paper included in this NISTIR is divided into Parts I, II, and III. Part I describes PC$1 \mathrm{D}$ from the point of view of high-accuracy photodiode modeling, and it also describes the programs that support its use in this application. The source code listed in the appendices to Part I could be easily modified to support other specialized applications of PC-1D. Parts II and III present examples of the use of the programs described in Part I to model two different types of experiments performed on silicon photodiodes in various high-accuracy applications.

\section{References}

[1] Geist, J., et al., Numerical Modeling of Silicon Photodiodes for High-Accuracy Applications, Parts I, II, and III, J. Res. NIST 96, 463-492 (1991).

[2] Basore, P. A., Proceedings of the 20th IEEE Photovoltaic Specialists Conference-1988, pub. \# 0160-8371/88/0000-0389 (IEEE, New York, 1988), p. 389.

* References to commercial software and equipment are provided throughout this paper to facilitate the complete description of the experimental procedures used, and constitute neither endorsements nor representations that the products so referenced are the best for the particular purposes. 


\title{
Part I: SIMULATION PROGRAMS
}

\author{
Jon Geist and Deane Chandler-Horowitz \\ NIST, Gaithersburg, MD 20899 \\ A. M. Robinson and C. R. James \\ University of Alberta, Alberta, Canada T6G 2G7
}

\begin{abstract}
The suitability of the semiconductor-device modeling program PC-1D for high-accuracy simulation of silicon photodiodes is discussed. A set of user interface programs optimized to support high-accuracy batch-mode operation of PC-1D for modeling the internal quantum efficiency of photodiodes is also described. The optimization includes correction for the dark current under reverse- and forward-bias conditions before calculating the quantum efficiency, and easy access to the highest numerical accuracy available from PC-1D, neither of which are conveniently available with PC-1D's standard user interface.
\end{abstract}

Key words: high accuracy; internal quantum efficiency; PC-1D; photodiode modeling; silicon photodiodes.

\section{Introduction}

PC-1D [1-2] is a computer program for numerical modeling of the electrical performance of one-dimensional semiconductor devices including photogeneration. It is optimized for solar cell modeling, and runs on IBM PC-compatible computers equipped with a numerical coprocessor. Version 2 of PC-1D includes realistic models of most of the semiconductor material and device properties that are important for high-accuracy applications of photodiodes. Consequently, Version 2 of PC-1D has the potential to be a tool for supporting these applications.

Unfortunately, for reasons that have to do with the difference between the applications for high-accuracy photodiodes and those for solar cells, it is not easy to achieve the highest accuracy of PC-1D through its standard user interface. Luckily, the designer of Version 2 of PC-1D anticipated this sort of problem and included the option to run it in batch mode in a way that does allow access to the highest levels of numerical precision and accuracy that are available from PC-1D.

This paper reports the development of a program shell for PC-1D that provides a batchmode user interface optimized for high-accuracy modeling of photodiodes. The shell consists of three programs. The first is a program that prepares the input for PC-1D, the second is a program that reads the output from PC- $1 D$, and the third is a MS-DOS batch file program that supervises the sequential execution of PC-1D and the other two programs.

The remainder of Part I of this paper reviews PC-1D and describes the new program shell for high-accuracy modeling of photodiodes. Typical applications are described in Parts II and III. 


\section{Description of PC-1D}

PC-1D is an interactive, graphical, semiconductor-device simulation program that solves the fully-coupled, drift-diffusion (electron and hole transport) equations in one dimension. Its principal limitations are that it supports no more than three regions of possibly different materials, and that it is a one-dimensional model with a maximum of 150 finite elements. Within these constraints, the program is very versatile. It is well beyond the scope of this paper to describe its various capabilities.

Both the IBM Pascal source code and executable code are available. The latter requires an IBM PC-compatible computer equipped with at least 512 Kbytes of random access memory (RAM), a $80 \mathrm{X} 87$ coprocessor chip, a CGA, VGA, or EGA compatible graphics adapter, and a matching display. To use the shell described in this paper, batch-mode operation is necessary which requires an MS-DOS compatible operating system (Version 3.1 or greater).

Only one of the interactive modes of PC-1D is of interest for the purposes of this paper, the one that allows the creation and saving of parameter files. Parameter files contain all of the information needed by PC-1D to model a specific, user-defined device. These files are stored in binary format under user-defined names with .PRM as their extension. Included in the parameter file are the names of other data files needed by PC-1D to model the device.

\subsection{Opening Menu}

Figure 1 shows the opening menu when PC-1D is run in the interactive mode. An overview of the options mentioned in this menu is appropriate for what follows. The first, second, and fourth options, "Proceed with Solution," "Preview / Examine," and "Output Graphs:" are used in the interactive modeling mode, and are of no concern here. The "Solution Mode:," "Base Voltage:," "Collector Voltage:," and "Light:" options are used to set the electrical and optical conditions under which the simulation will be run. For the purposes of this paper, it does not matter what selections are made for these options, because they will be set to the correct values by the high-accuracy photodiode modeling shell when it is run.

The "Simulation Parameters:" option is used to load parameter files in the interactive mode, but it is also used to save parameter files, which is of interest to the use of PC1D in batch mode. The "Reinitialize:" and "Number of Finite Elements:" options allow details of the numerical procedures to be modified to a certain extent. Normally, 150 finite elements, the maximum allowed, will be needed for high-accuracy modeling. It will sometimes be necessary to use this menu option in order to force PC-1D to use all 150 elements. Reinitialization is probably desirable except, perhaps, when simulating a reverse-bias experiment.

The remaining options define the detailed nature of the device and external circuit to be modeled. All of the parameters obtained from the internal models built into PC-1D are adjusted to the values appropriate for the temperature entered following the "Temperature:" heading at the bottom of the opening menu. The user, of course, must assure that 


\section{PC-1D PROGRAM CONIROL}

Proceed with Solution

Treviev / Ixamine (None)

Simulation Parameters: 1337_NEV

Output Graphs: Specified

Reinitialize: Yes

Number of Tinite Elements: 150

Solution Mode: Equilibrium

lemperature: 256

Area: Uniform $1 \mathrm{~cm}$

Thicknesses: 1 uh, 299 un

Naterials: \$1*, \$I*

Doping: 1337, Internal

Reconbination' Internal

Surf aces: Charged, Neutral

Circuit: Vhb

Base Vol tage: Zero

Collector Voltage: Zero

Light: 10 uH/GM2

Photogeneration: Monochrome $440 \mathrm{~nm}$

Reflectance: $0,0.85$

Figure 1. The opening menu of $\mathrm{PC}-1 \mathrm{D}$ when run in the interactive mode. 
any user-defined parameters or data fles are consistent with the chosen temperature. The "Area:" option is straightforward. The "Thickness:" option allows three different regions to be defined, each having its own thickness, material, and doping properties. If the thickness of a region is set to zero, it is not included in the solution, and it is not explicitly indicated following the "Thickness:" heading.

\subsubsection{Materials Properties}

Selection of the "Materials:" heading of the opening menu allows such properties as the carrier mobilities, band-gap narrowing, Auger recombination cross sections, and absorption coefficient to be adjusted. For each region, a set of three files having the extensions.MAT, .INR, and .ABS are needed. These files are 1) an ASCII file that is the main material file, 2) an ASCII file containing wavelength versus real index of refraction data, and 3 ) an ASCII file containing wavelength versus absorption-coefficient data, respectively. All of the file names are stored in the parameter (PRM) file. In default operation, the INR and ABS files will have the same name as the MAT file. The user has the option of associating alternate ABS and INR files with a given MAT file in a particular parameter file, even though they have different names. If this is done, asterisks follow the MAT file names in the "Materials:" option heading, as shown in figure 1. Other material properties are included in internal models. Some of these allow the user to specify the values of the parameters in the equations defining the models, and most can be replaced by user-supplied ASCII files having the appropriate extensions. The internal model of the absorption coefficient of silicon, which is discussed next, is an example.

The default absorption-coefficient data in the SI.ABS file of Version 2 of PC-1D are not accurate enough for high-accuracy modeling of silicon photodiodes over the 400 to $900 \mathrm{~nm}$ spectral region [3]. Therefore, two data sets of higher accuracy were calculated and stored in files named SIL_WEAK.ABS and SIL_PHIL.ABS. The first data set was calculated by using eq (1) of Ref. [3], which was fitted to the data of Ref. [4] over the spectral range from 470 to $1180 \mathrm{~nm}$. The second data set was calculated by interpolation of the data of Ref. [5]. The first set is expected to be more accurate at longer wavelengths, and the second at shorter wavelengths.

The ratios of the data in SIL_PHIL.ABS to that in SIL_WEAK.ABS are plotted over the 400 to $900 \mathrm{~nm}$ spectral range in figure 2. The difference can be characterized as $12 \% \pm$ $4 \%$ over the spectral region from 440 to $780 \mathrm{~nm}$, growing much larger outside that spectral region. The value of $12 \%$ is a convenient average offset because it is the difference between the two data sets at $633 \mathrm{~nm}$. At that wavelength, the value calculated from eq (1) of Ref. [3] agrees with a recent measurement [6] to within the $\pm 2 \%$ uncertainty associated with the measurement.

One material property not modeled by PC-1D is the quantum yield for electron hole pair production [7]. This quantity may differ significantly from unity outside the 400 to 900 nm spectral region [8], but no high accuracy models currently exist $[7,9]$. This is the main reason that the modeling described in Part II of this paper is confined to the 400 to 900 $\mathrm{nm}$ spectral region. However, other problems, such as larger uncertainties in the available 


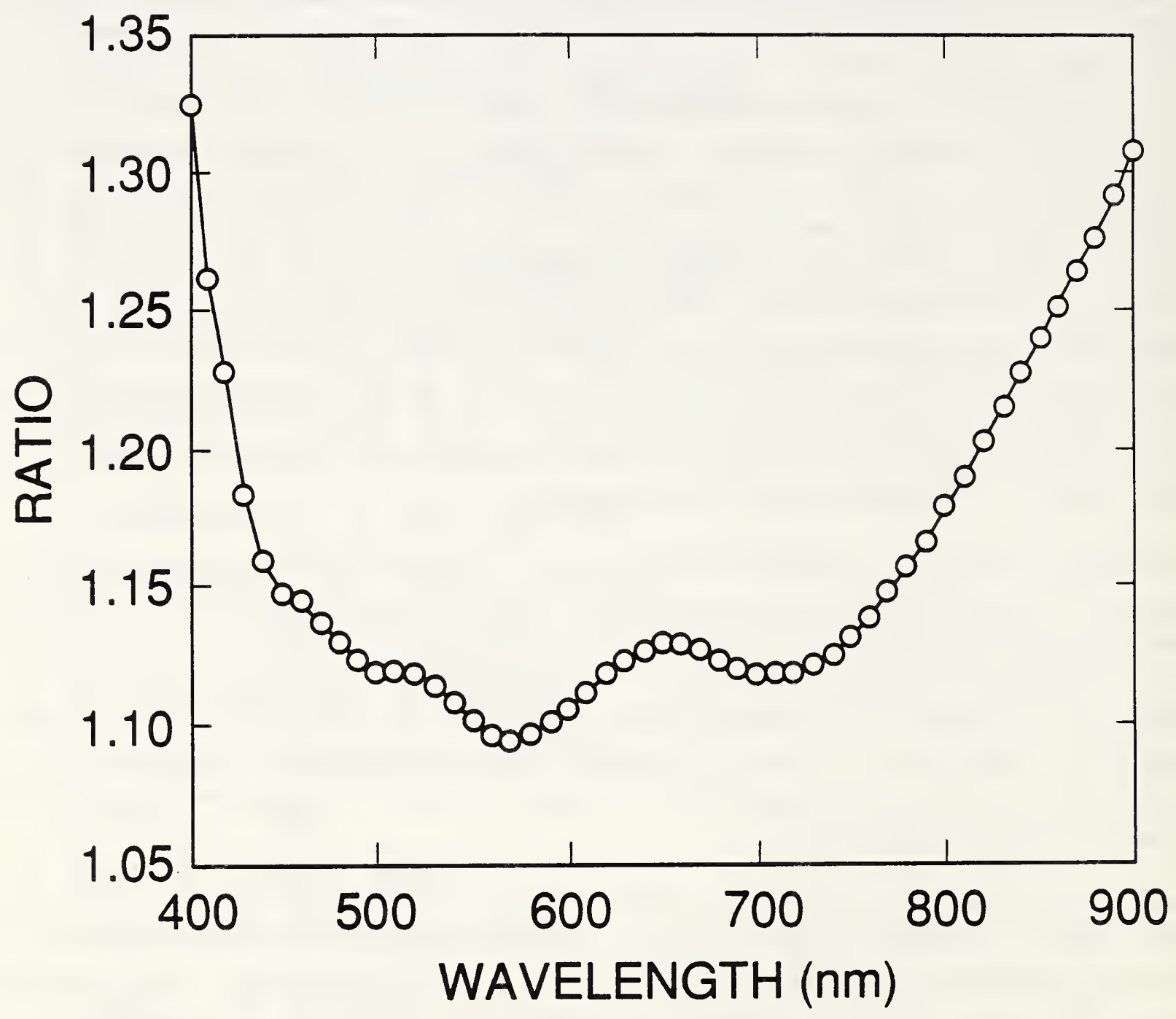

Figure 2. Ratios of the absorption-coefficient data interpolated from Ref. [5] to those calculated from eq (1) of Ref. [3] that were fitted to the data of Ref. [4]. 
absorption-coefficient data at shorter wavelengths and uncertainties in the fraction of the radiation reflected by the rear surface of the photodiode at the longer wavelengths, also contribute to a significant deterioration in accuracy outside that spectral region.

\subsubsection{Device Properties}

The "Doping:" heading of the opening menu of PC-1D allows either an internal doping model or an external file containing doping concentrations for each region of the device being modeled. The internal model consists of two front and two rear dopant distributions, as well as a uniform background dopant. The distributions can be chosen from uniform, complementary error, and Gaussian functions, and the parameters defining the maximum value, its location, and the width of the distributions can be adjusted. If tabular doping data are to be used, they are read from a user-generated ASCII file containing a depth, an n-type dopant concentration, and a p-type dopant concentration on each line, and having .DOP as its extension.

The shape of the equilibrium majority-carrier concentration near the front-surface oxidesilicon interface is very important to high-accuracy photodiode modeling [10]. It is necessary to use external files to model the front region doping in $\mathrm{p}^{{ }^{+}} \mathrm{n}$ type photodiodes to force $\mathrm{PC}-1 \mathrm{D}$ to devote enough finite elements to the front region to approximate accurately the majority-carrier concentration there.

The "Recombination:" heading of the opening menu of PC-1D allows the defect-related recombination in the volume and at the surfaces of the device to be modeled. (Auger recombination is considered a material property and is covered under the "Materials:" heading.) Shockley-Read-Hall (SRH) recombination through a single energy-level trap state is used as the model for volume recombination. Either an internal model or a userdefined table of depths and electron and hole lifetimes (equivalent to cross sections in the SRH model) in an external ASCII file with the extension. TAU can be used to model the volume recombination. With the internal model, the user may choose a single electron lifetime, a single hole lifetime, and a single trap energy relative to mid-gap for each region. With the external model, a trap level at mid-gap is used with the lifetime data in the external ASCII file.

The restriction of the SRH model to a single-energy state prevents PC-1D from accurately fitting the measured [11] variation of quantum efficiency with flux (i.e., nonlinearity) at $950 \mathrm{~nm}$ for an EG\&G UV444B photodiode as illustrated in figure 3 . The simulated data in figure 3 were calculated using a mid-gap state with equal electron and hole lifetimes. Even though the simulated and experimental data agree in the low flux limit and again at a high flux level, their shapes are very different. Better agreement can be achieved at low flux levels at the cost of a worse fit at the higher flux levels by moving the state away from mid-gap, and by using different electron and hole lifetimes as illustrated in figure 4 . It is expected that the proper distribution of SRH trap levels over the band gap would result in an accurate simulation of the nonlinearity of this type of photodiode.

It should be possible to develop an external lifetime model based on a parameterized dis- 


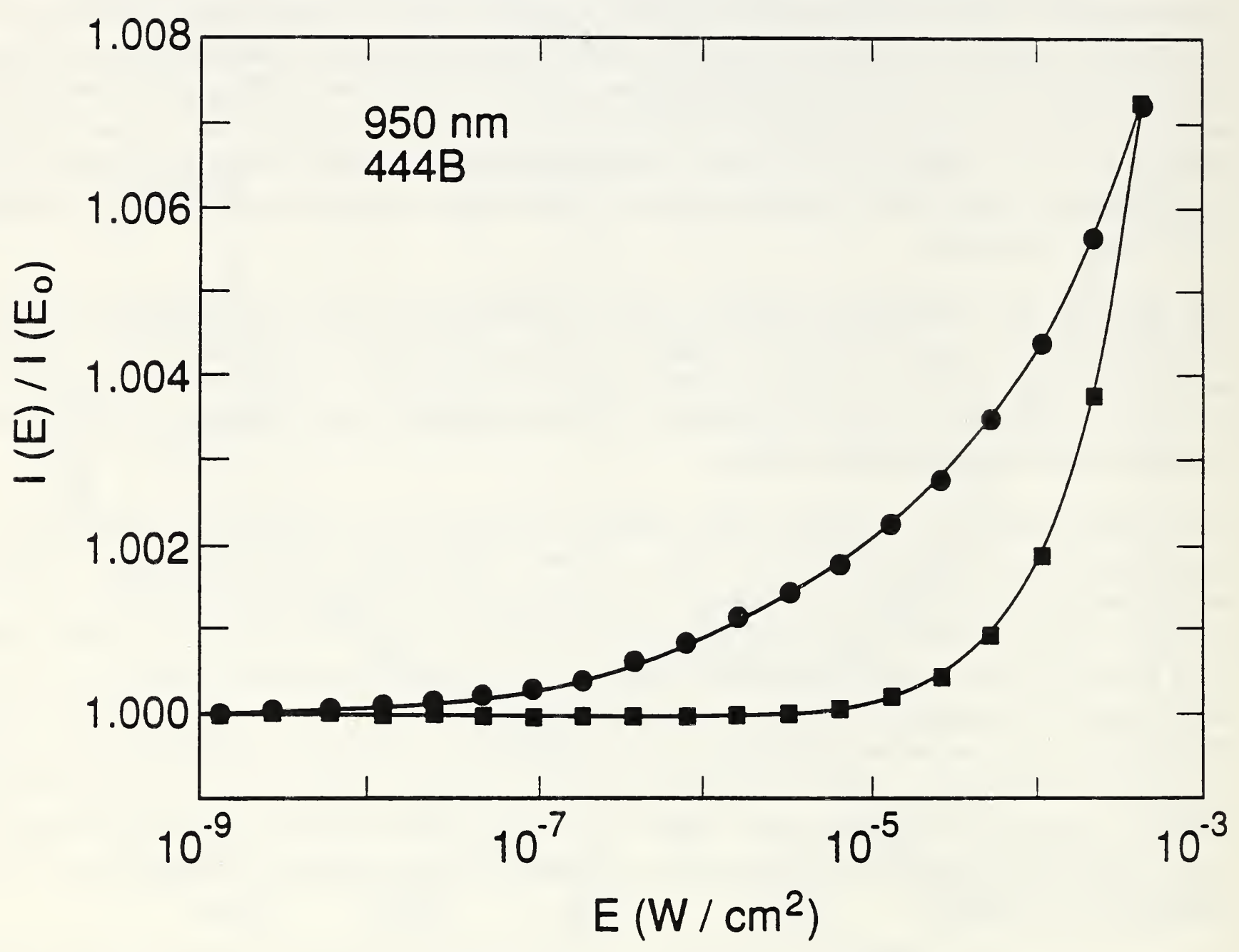

Figure 3. Comparison of experimental (filled circles) and simulated (filled squares) linearity measurements on an EG\&G UV444B photodiode at $950 \mathrm{~nm}$ using a single SRH trap level at mid-gap with electron and hole lifetimes of $74.04 \mu \mathrm{s}$. 


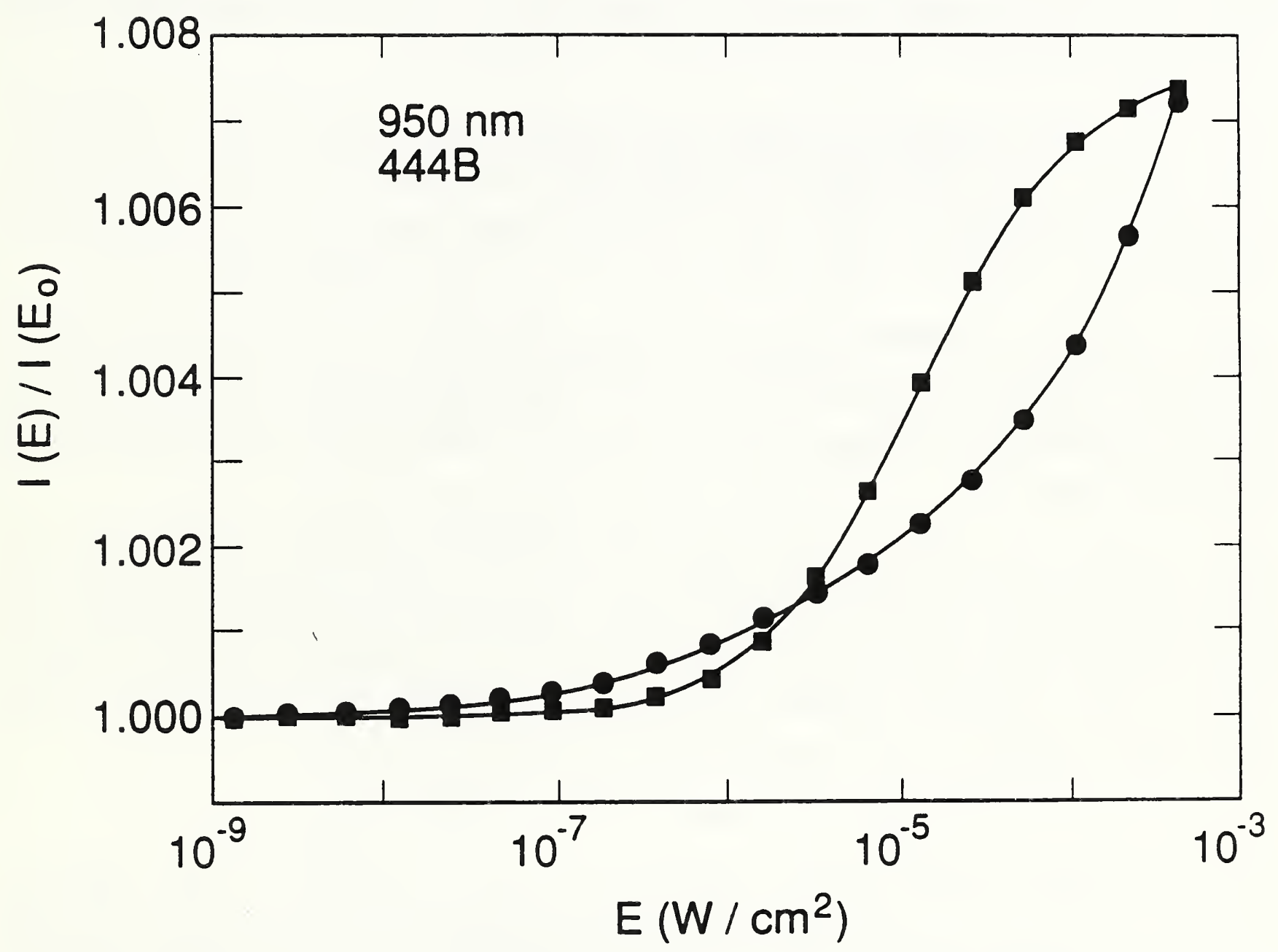

Figure 4. Comparison of experimental (filled circles) and simulated (filled squares) linearity measurements on an EG\&G UV444B photodiode at $950 \mathrm{~nm}$ using a single SRH trap level $0.1796 \mathrm{eV}$ above mid-gap with electron and hole (check for vice versa) lifetimes of $900 \mathrm{~ms}$ and $700 \mathrm{~ms}$, respectively. 
tribution of SRH recombination states, and to adjust the parameters to fit the nonlinearity data shown in figures 3 and 4 . However, it would be necessary alternately to run PC-1D to calculate the carrier concentrations and then the external model to calculate the lifetimes appropriate to these carrier concentrations, and to iterate to self-consistency. This is beyond the scope of the work reported in this paper.

The failure of the model to describe the nonlinearity of this type of photodiode casts some doubt on its ability to model accurately the reverse-bias self-calibration experiment [12-13]. This point is addressed in more detail in Part II of this paper.

In the case of surface recombination, the "Recombination:" option allows the choice of one of two internal models, a surface-recombination velocity model, and a saturationcurrent density model, but does not accommodate a user-defined data file. The choice of the surface-recombination velocity model allows the user to adjust the hole- and electronrecombination velocities as well as the energy-level of a single-energy surface state.

The "Surfaces:" option allows the user to choose the surface charge at the front and back surfaces of the device. For modeling the oxide-bias self-calibration experiment [12-13], the surface charge is the algebraic sum of any charge trapped in the oxide as a result of thermal oxidation and the charge stored on the oxide surface by the voltage applied to the transparent electrode.

The "Circuit:" option of the main menu of PC-1D allows the user to define a circuit in which the device is to operate. For the case of a photodiode, the emitter and base are connected, the collector is disconnected, and the connections to the device are made at the front and back surface. Keeping the collector disconnected forces the "Collector Voltage:" option to zero, as shown in figure 1 . Setting the internal resistance $R_{b}$ to some non-zero value allows the effect of series resistance to be simulated.

\section{The Photodiode Modeling Shell Programs}

The photodiode modeling shell for Version 2 of PC-1D is designed to allow simulation of oxide-bias and reverse-bias, self-calibration experiments, nonlinearity measurements, and internal quantum efficiency spectra, while accessing the highest accuracy and precision available from PC-1D. It consists of three program files. The first program, a short MS-DOS Batch program named RUN.PC1D.BAT, is listed in figure 5. The other two programs, MAKE_PRM.EXE and READ_PDF.EXE, were complied with Version 5.5 of

Turbo Pascal. Listings of the source code for these programs are given in Appendices I and II.

\subsection{Operation of RUN_PC1D.BAT}

The first thing that RUN.PC1D.BAT does when run is to test whether or not the files MAKE_PRM.EXE, PC-1D.EXE, and READ_PDF.EXE all exist. If not, it issues an error message, and terminates execution at :STOP. If all three files do exist, RUN_PC1D deletes the temporary file TEMP.DAT. This is a precaution in case a previous nonstandard termination left this file in existence. RUN_PC1D then enters the loop between :LOOP and 


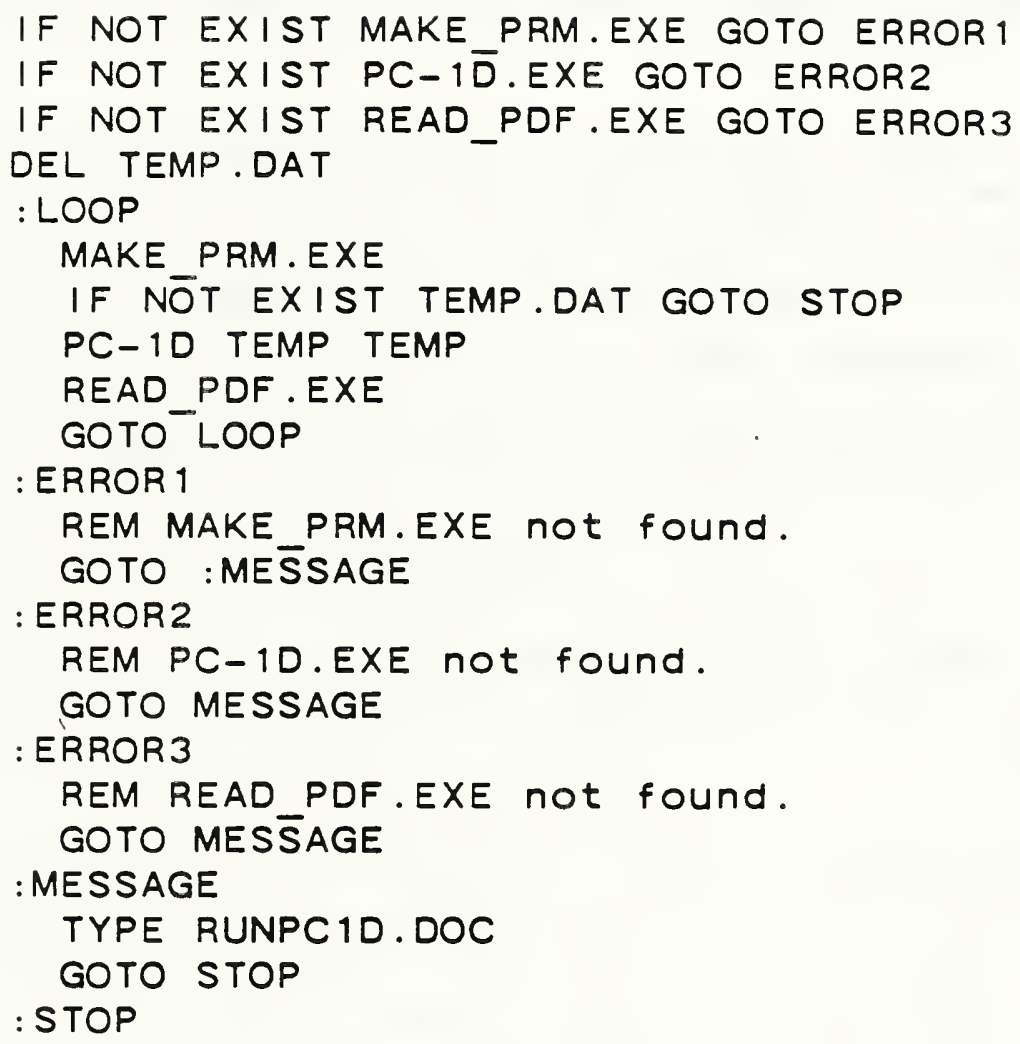

Figure 5. Listing for the MSDOS batch file RUN PC1D.BAT that supervises the execution of the programs MAKE_PRM.EXE, PC-1D.EXE, and READ_PDF.EXE for high-accuracy photodiode modeling. 


\section{GO TO LOOP. Once in this loop, it transfers control to MAKEPRM.EXE.}

The first thing that MAKE_PRM.EXE does is to test the existence of TEMP.DAT. If TE.MP.DAT does not exist, then MAKE PRM.EXE prompts the user to define the photodiode experiment to be simulated. Examples of the questions and typical answers are shown in Appendix $A$. The questions and answers are straightforward and require no explanation. If the file option rather than the keyboard option is chosen to define the independent variables for the experiment to be simulated, then the program asks for the file name instead of the start, stop, and step values for the independent variable. Once MAKE_PRM.EXE has obtained all of the necessary information, it writes that information into the file TEMP.DAT, creates the temporary parameter file TEMP.PRM, and passes control back to RUN.PC1D.BAT.

RUN_PC1D.BAT now tests the existence of TEMP.DAT. If it does not exist, RUN PC1D terminates execution. If TEMP.DAT does exist, then RUN PC1D passes control to PC1D.EXE. This program reads TEMP.PRM (the first TEMP on the command line following PC-1D), carries out the simulation defined therein, writes a table having a depth, an electron current, a hole current, and a total current on each line in the file TE.MP.PDF (the second TEMP on the command line), and returns control to RUN_PC1D.BAT. Note that PDF files are the standard ASCII format HP plotter output files generated by PC-1D.

RUN_PC1D.BAT now passes control to READ_PDF.EXE. This program reads the total photocurrent at the depth specified in the definition of the experiment to be simulated (see Appendix A) from file TEMP.PDF, and appends it to the output file specified in response to the prompt from MAKE_PRM.EXE. READ_PDF.EXE then returns control to RUN_PC1D.BAT.

On subsequent passes through the loop, MAKE_PRM.EXE finds that TEMP.PRM does exist, reads the necessary information from this file, and writes updated TEMP.DAT and TEMP.PRM files. The information about when to exit the loop is in TEMP.DAT, and when MAKE_PRM.EXE determines that this time has come, it deletes TEMP.DAT, triggering RUN_PC1D.BAT to terminate execution.

\subsection{Features of MAKE_PRM and READ_PDF}

A few points about MAKE_PRM.EXE and READ_PDF.EXE are in order. First, these two programs are designed to compensate for traits of PC-1D that make it less than ideal for high-accuracy modeling of photodiodes. The first of these is that PC-1D.EXE calculates the internal quantum efficiency from the sum of the photocurrent and the dark current occurring under reverse or forward bias. This may not be a problem in solar cell modeling, but it is not correct for photodiode modeling. As a result, MAKEPRM.EXE calculates and stores the appropriate dark currents for all simulations that have a nonzero bias voltage $V_{b b}$, and READ_PRM.EXE reads them and subtracts them from the appropriate total currents when calculating the quantum efficiency. This is simulated as a shutterclosed/shutter-open measurement sequence by MAKE_PRM.EXE.

Even though the total current is a constant throughout a photodiode, it must be calculated 
at each finite element in the photodiode. The numerical precision with which it can be calculated depends upon the majority-carrier concentration in the element where it is being calculated due to nature of the numerical algorithm, which involves the difference of two large numbers that scale with doping concentration. This is illustrated in figure 6 , which shows the variation in the total current computed by $\mathrm{PC}-1 \mathrm{D}$ as a function of depth in the front region of a $\mathrm{p}^{+} \mathrm{n}$ type photodiode irradiated by $1 \mu \mathrm{W} / \mathrm{cm}^{2}$ of $440 \mathrm{~nm}$ radiation. This result was obtained by running $\mathrm{PC}-1 \mathrm{D}$ through its standard user interface and could not be obtained by running PC-1D with the shell just described, since carrier concentration as a function of depth is not an output supported by the shell. The decrease in the noise in the data with increasing distance from the front surface of the photodiode is evident in the figure.

PC-1D.EXE calculates the quantum efficiency from the total current at either the front or the rear of the photodiode at the discretion of the user. Since these are usually the regions where the majority-carrier concentration is greatest, PC-1D often calculates the quantum efficiency from values of the total photocurrent that are not the most precise available to it. This loss of precision, which occurs even in the absence of dark current, is the reason that MAKEPRM.EXE asks the user to choose the depth at which the total photocurrent will be read from the TEMP.PDF file. This depth should be somewhere in the interior of the photodiode where the doping concentrations are low and the effect of surface fields on the carrier concentrations is small or negligible.

Very high precision is available under these conditions. The internal quantum efficiency calculated by RUN_PC1D for the same type of photodiode used to generate the data in figure 6 , but with all loss (recombination) mechanisms set to zero, is 6 parts per million $(\mathrm{ppm})$ above unity $\pm 1 \mathrm{ppm}$ for $1 \mu \mathrm{W} / \mathrm{cm}^{2}$ irradiation anywhere within 400 to $900 \mathrm{~nm}$ spectral region. This result was obtained by assuming the somewhat large thickness of 400 $\mathrm{mm}$ for this photodiode to minimize the fraction of the incident radiation penetrating to the rear of the photodiode. Since PC-1D does not permit a reflectance of $100 \%$ at the rear surface of the diode, a more typical 300-mm thick photodiode would show a loss of a few ppm at the longest wavelengths.

Since PC-1D does not model the oxide passivation on the front surface of the photodiode, it is necessary to vary the oxide trapped charge $\mathrm{N}_{\text {. }}$ to simulate the effect of applying a voltage across the oxide in the silicon self-calibration experiment. First, the value of $\mathrm{N}_{s}$ must be chosen to simulate the oxide-fixed charge. To this value must be added the charge appropriate to the application of oxide bias according to the formula for the charge density stored on a capacitor as a function of thickness, dielectric constant, and applied voltage.

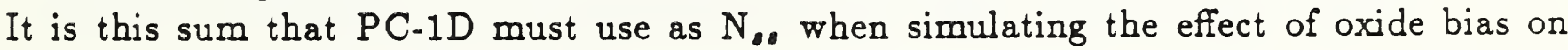

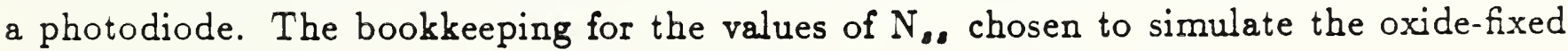
charge and the values of $N_{\text {, }}$, needed to simulate the combined effect of the fixed charge and the applied oxide bias are handled by MAKEPRM.EXE. 


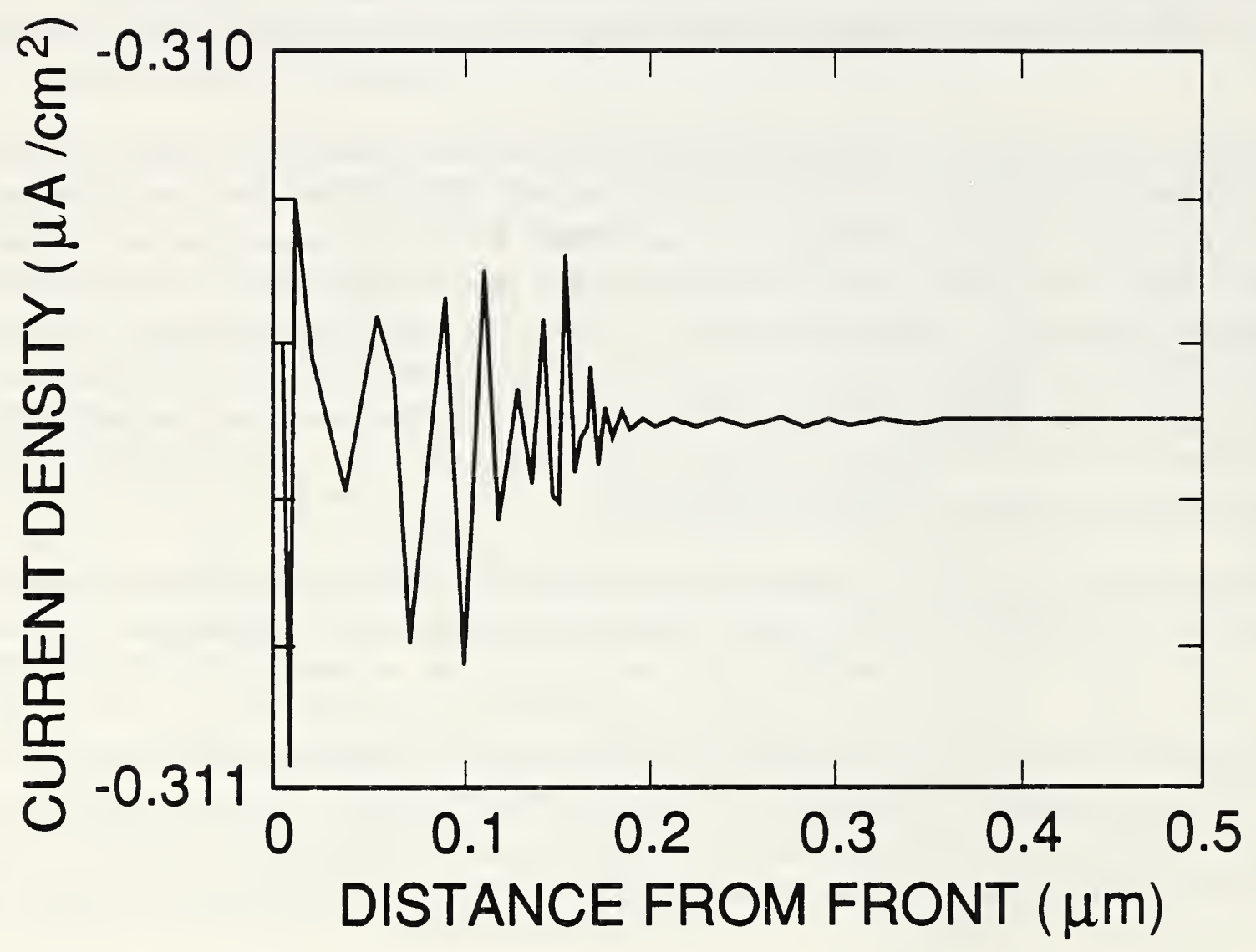

Figure 6. Variation in the total photocurrent calculated by PC-1D as a function of position in the front region of a Hamamatsu 1337 type photodiode due to the effect of the high front region doping concentration. 


\section{Conclusion}

The semiconductor device modeling program PC-1D and some programs to enhance its use in the high-accuracy modeling of silicon photodiodes have been described. The features of PC-1D that suit it for high-accuracy photodiode modeling have been described, and the features of the support programs that compensate for features of the PC-1D user interface that are less than ideal for this application have also been described. Examples of the use of these programs in photodiode modeling in different types of high-accuracy applications are given in Parts II and III of this paper.

5. References

[1] Available from ISU Extension Software, 108 Atanasoff Hall, Iowa State University, Ames, IA 50011, (505) 294-8658.

[2] Basore, P. A., Proceedings of the 20th IEEE Photovoltaic Specialists Conference-1988, pub. \# 0160-8371/88/0000-0389 (IEEE, NY, 1988), p. 389.

[3] Geist, J., Migdall, A., and Baltes, H. P., Appl. Opt. 27, 3777 (1988).

[4] Weakliem, H. A., and Redfield, D., J. Appl. Phys. 50, 1491 (1979), and personal communication from H. A. Weakliem.

[5] Philipp, H. R., J. Appl. Phys. 43, 2835 (1972), and personal communication.

[6] Geist, J., Schaefer, A. R., Song, J-F., Wang, Y. H., and Zalewski, E. F., J. Res. NIST 95, 549 (1990).

[7] Geist, J., and Wang, C. S., Phys. Rev. B27, 4841 (1983).

[8] Geist, J., Gladden, W. K., and Zalewski, E. F., J. Opt. Soc. 72, 1068 (1982).

[9] Geist, J., Gardner, J. L., and Wilkinson, F. J., Phys. Rev. B42, 1262 (1990).

[10] Geist, J., and Baltes, H., Appl. Opt. 26, 3929-3939 (1989). Note that there is an error in eq (31) of this reference. The plus sign in the numerator of the first term in eq (31) should be replaced by a minus sign.

[11] Stock, K. D., Appl. Opt. 25, 830 (1986), and personal communication.

[12] Zalewski, E. F., and Geist, J., Appl. Opt. 19, 1214 (1980).

[13] Geist, J., Zalewski, E. F., and Schaefer, A. R., Appl. Opt. 19, 3795 (1980). 
APPENDIX I. A listing of MAKE_PRM.PAS that compiles under Turbo Pascal Version 5.5

PROGRAM make_temporary_prm_files_for_running_pcld;

\{RUN_PC1D.BAT runs this program when this program is named MAKE_PRM.EXE.

This program writes 2 temporary files, TEMP.PRM and TEMP.DAT.

RUN_PC1D.BAT then runs PC-1D with TEMP.PRM as input and TEMP.PDF as output.

RUN_PC1D.BAT then runs READ_PDF.EXE.

READ_PDF.EXE uses TEMP.PDF and TEMP.DAT to write $\mathrm{QE}$ data to a file.

RUN_PC1D.BAT runs MAKE_PRM.EXE again.

MAKE_PRM.EXE uses TEMP.DAT to decide to either

1) increment some parameters in TEMP.DAT and TEMP.PRM, or

2) delete the 3 temporary files.

$\{\$ N+\}$

USES CRT, DOS;

TYPE file_of_values_type $=$ FILE OF DOUBLE;

TYPE p_index_type $=$

(I_parameters, I_max_iterations,

I_mode, I_graphs, I_size_factor, I_error_limit,

I_clamp, I_step_size, I_final_step,

I_solutions_step, I_eq_renode, I_ss_renode, I_tr_renode,

I_graph_1, I_graph_2, I_graph_3, I_graph_4,

I_area, I_area_value, I_facet_depth, I_facet_angle,

I_temperature, I_temp_units,

I_front_surface, I_back_surface,

I_generation, I_front_texture, I_back_texture,

I_int_refl_value, I_front_refl,

I_front_refl_value, I_back_refl_value,

I_front_n1, I_front_d1,

I_front_n2, I_front_d2,

I_front_n3, I_front_d3,

I_front_barrier, I_back_barrier,

I_front_Nss, I_back_Nss,

I_base_location, I_emitter_location, I_collector_location,

I_shunt_loc1, I_shunt_loc2,

I_band_file, I_boundary_dx, I_recombine,

I_collector, I_base, I_shunt,

I_Vcc_shape, I_Vcc_start, I_Vcc_end,

I_Vbb_shape, I_Vbb_start, I_Vbō_end,

I_light_shape, I_light_start, I_light_end,

I_spectrum, I_lambda_start, İ_lamb̄a_end,

I_Rcc, I_Rbb, I_Rc, I_Rb, 
I Gb, I Gc, I Gsh,

I_Db, I_Dc, I_Nb, I_Nc);

q_index_type $=$

(I_w, I_material, I_material_mod,

I_doping, I_recomb_front, I_recomb_back,

I_taun, I_taup, I_Et,

I_Sn_front, I_Sp_front, I_Et_front,

I_Sn_back, I_Sp_back, I_Et_back,

I_J0_front, I_J0_front_n, I_J0_back, I_J0_back_n,

I_Nbjk, I_Nbj_type,

I_Nf1, I_Nf1_type, I_Nf1_shape, I_Nf1_depth, I_Nf1_peak,

I_Nf2, I_Nf2_type, I_Nf2_shape, I_Nf2_depth, I_Nf2_peak,

I_Nr1, I_Nr1_type, I_Nr1_shape, I_Nr1_depth, I_Nr1_peak,

I_Nr2, I_Nr2_type, I_Nr2_shape, I_Nr2_depth, I_Nr2_peak,

I_Eg, I_affinity, I_NcNv, I_ni0, I_eps,

I_Cnt, I_Cpt, I_Cn, I_Cp, I_Bn, I_Bp,

I_BGN_Phid, I_BGN_Nd, I_BGN_Phia, I_BGN_Na,

I_n_file,

I_n,

I_mu_max_n,

I_mu_min_nn, I_Nref_nn, I_alpha_nn,

I_mu_min_np, I_Nref_np, I_alpha_np,

I_mu_nbl, I_mu_nb2, I_mu_nb3, i_mu_nb4,

I_mu_max_p,

I_mu_min_pp, I_Nref_pp, I_alpha_pp,

I_mu_min_pn, I_Nref_pn, I_alpha_pn,

I_mu_pbl, I_mu_pb2, I_mu_pb3, i_mu_pb4,

I_n_sat, I_p_sat,

I absorb file,

I_Egd1, I_Egd2, I_Eg_1, I_Eg2, I_Ep1, I_Ep2,

I_Asb_Ad1, I_Abs_Ad2,

I_Abs_A1, I_Abs_A2, I_Abs_A3, I_Abs_A4,

I_Abs_B, I_Ābs_T̄ $\mathrm{T} g$ );

variant_type $=($ str_16, byt_16, rea_08, int_04 $)$;

s_type = STRING[15];

b_type = ARRAY[0..15] OF BYTE;

r_type = ARRAY[0..1] OF DOUBLE;

i_type $=$ ARRAY[0..3] OF LONGINT;

P_type $=$ RECORD

CASE variant : variant_type $O F$

str_16:(s : ARRAY[p_index_type] OF s_type);

byt_16 : (b : ARRAY[p_index_type] OF b_type);

rea_08 : $(r:$ : ARRAY[p_index_type] OF r_type);

int_04 : (i : ARRAY[p_index_type] OF i_type); 


\section{END;}

q_type $=$ RECORD

CASE variant : variant_type OF

str_16:(s : ARRAY[1..3] OF ARRAY[q_index_type] OF s_type);

byt_16 : (b : ARRAY[1..3] OF ARRAY[q_index_type] OF $\bar{b}_{-}$type);

rea_08 : ( $r$ : ARRAY[1..3] OF ARRAY[q_index_type] OF r_type);

int_04 : (i : ARRAY[1..3] OF ARRAY[q_index_type] OF i_type);

END;

TYPE prm_data_type

$=$ OBJECT

model_prm_file_path : PATHSTR;

$\mathrm{p}: \mathrm{p}$ _type;

q : q type;

PROCEDURE read_prm_file; \{page \}

PROCEDURE write_prm_file; \{page \}

END;

TYPE exp_data_type

$=$ OBJECT(prm_data_type)

key : CHAR;

shutter : STRING[6];

lambda, energy, phi, Vo, Vr, depth, oxide_t, value : DOUBLE;

index, last : LONGINT;

QE file_path : PATHSTR;

FUÑCTION file_exists( path : PATHSTR) : BOOLEAN; \{page \}

PROCEDURE initialize_or_update;

PROCEDURE initialize;

PROCEDURE get_parameter_to_scan;

PROCEDURE get_values_from_file;

$\{O R\}$

PROCEDURE get_start_stop_and_step;

PROCEDURE set_parameter_to_value;

PROCEDURE open_empty_QE_file;

PROCEDURE write_temp_data;

PROCEDURE update;

PROCEDURE get_current_value;

\{set_parameter_to_value;

\{write_temp_data; $\}$

PROCEDURE prepare_pq;

PROCEDURE delete_temporary_files;

END;

\{page

\{page

\{page

\{page

\{page

\{page

\{page

\{page

\{page $\}$

\{page \}

\{page \}

VAR exp_data : exp_data_type;

FUNCTION exp_data_type.file_exists( path : PATHSTR) : BOOLEAN;

VAR f : TEXT; 


\section{BEGIN}

$\{\$ I-\}$

ASSIGN(f, path);

$\operatorname{RESET}(\mathrm{f})$;

CLOSE $(\mathrm{f})$;

$\{\$ I+\}$

IF $($ IORESULT $=0)$

THEN file_exists $:=$ TRUE

ELSE file_exists $:=$ FALSE;

END;

PROCEDURE exp_data_type.initialize_or_update;

VAR f : TEXT;

BEGIN

IF NOT file_exists( 'temp.dat' )

THEN BEGIN

CLRSCR;

IF file_exists( 'J0.dat' )

THEN BEGIN

$\operatorname{ASSIGN(f,~'J0.dat'~);~}$

$\operatorname{ERASE}(\mathrm{f})$

END;

WRITELN (

'Parameter file (including PRM extension) defining photodiode: ');

READLN(model_prm_file_path);

IF NOT file_exists(model_prm_file_path )

THEN BEGIN

WRITELN('File: ', model_prm_file_path, ' not found. Halting.');

HALT;

END;

WRITELN('Complete path to file in which to store QE results: ');

READLN(QE_file_path);

initialize;

END

ELSE BEGIN

update;

END;

END;

PROCEDURE exp_data_type.initialize;

VAR $s$ : STRING[1];

BEGIN

get_parameter_to_scan;

$\mathrm{s}:=$ ' ';

WHILE NOT ( UPCASE(s[1]) IN ['F', 'K'] )

DO BEGIN

WRITE(

'Start, step, and stop values from (K)eyboard or all values from (F)ile? '); 


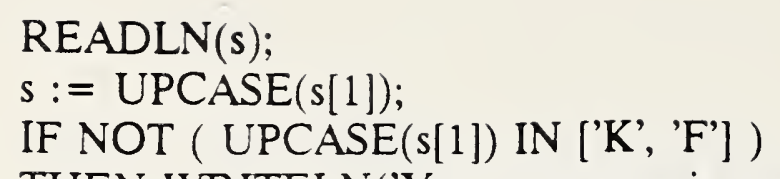

IF key $<>$ ' $L$ '

THEN BEGIN

WRITE('Incident irradiance (W per sq. cm): ');

READLN(phi);

END;

IF key <> 'O'

THEN BEGIN

WRITE('Oxide bias voltage (V): ');

READLN(Vo);

END;

IF key $<>$ 'R'

THEN BEGIN

WRITE('Reverse bias voltage (V): ');

READLN(Vr);

END;

WRITE('Non-zero thickness of front surface oxide (nm): '); READLN(oxide_t);

WRITE('Depth at which to get total photocurrent (um): '); READLN(depth);

IF $V_{r}=0$

THEN shutter $:=$ 'open'

ELSE shutter := 'closed';

open_empty_QE_file;

write_temp_data;

END;

PROCEDURE exp_data_type.get_parameter_to_scan;

TYPE letter_set_type = SET OF 'A' .. ' $Z$ ';

CONST answer_set : letter_set_type $=\left[{ }^{\prime} O\right.$ ', 'R', 'W', 'P', 'L'];

VAR s : STRING[1];

BEGIN

$\mathrm{s}:=$ ' ';

WHILE NOT ( UPCASE(s[1]) IN answer_set )

DO BEGIN

\{CLRSCR;\}

WRITELN('Type of experiment to simulate? '); 
WRITELN('(O)xide bias (Volts) scan? ');

WRITELN('(R)everse bias (Volts) scan?');

WRITELN('(W)avelength (nm) scan?' ');

WRITELN ('(P)hoton energy (eV) scan? ');

WRITELN('(L)inearity (W/cm ^ ${ }^{\wedge}$ ) scan? ');

WRITE('Enter letter key here: '); READLN(s);

IF NOT ( UPCASE(s[1]) IN answer_set )

THEN WRITELN('Your response is unacceptable. Try it again.');

END;

key := UPCASE $(\mathrm{s}[1])$;

END;

PROCEDURE exp_data_type.get_values_from_file;

VAR start, stop, step : DOUBLE;

name : STRING[80];

f : TEXT;

$\mathrm{g}:$ file_of_values_type;

\section{BEGIN}

WRITE('Name of file with all values of scanned parameter: '); READLN(name);

ASSIGN(f, name);

$\operatorname{RESET}(\mathfrak{f})$;

$\operatorname{ASSIGN(g,~'temp.VAL');~}$

REWRITE(g);

READLN(f, start);

WRITE (g, start);

last $:=0$;

WHILE NOT EOF(f)

DO BEGIN

READLN(f, stop);

WRITE(g, stop);

INC(last);

END;

WRITE(g, stop);

INC(last);

CLOSE(f);

CLOSE (g);

index $:=0$;

value $:=$ start;

set_parameter_to_value;

END;

PROCEDURE exp_data_type.get_start_stop_and_step;

VAR start, stop, step : DOUBLE;

$\mathrm{g}:$ file_of vvalues_type;

\section{BEGIN}

WRITE('First value for scanned parameter: '); READLN(start);

WRITE('Stop value for scanned parameter: '); READLN(stop);

IF key $=$ 'L' 


\section{THEN BEGIN}

WRITE('Factor by which to increase irradiance on each iteration: ');

READLN(step);

IF ( (start >stop) AND (step $>=1))$

OR ( (start <stop) AND (step $<=1))$

THEN BEGIN

WRITELN('These values would produce an endless loop. Halting.');

HALT;

END;

\section{END}

ELSE BEGIN

WRITE('Step size for scanned parameter: ');

READLN(step);

IF ( (start >stop) AND (step $>=0))$

OR ( (start<stop) AND (step $<=0))$

THEN BEGIN

WRITELN('These values would produce an endless loop. Halting.');

HALT;

END;

END;

$\operatorname{ASSIGN(g,~'temp.VAL');~}$

REWRITE(g);

last : $=0$;

IF key $=$ 'L'

THEN BEGIN

value := start/step;

IF start < stop

THEN WHILE value $<=$ stop

DO BEGIN

value := value ${ }^{*}$ step;

WRITE(g, value);

INC(last);

END

ELSE WHILE value $>=$ stop

DO BEGIN

value $:=$ value step;

WRITE(g, value);

INC(last);

END;

END

\section{ELSE BEGIN}

value := start-step;

IF start < stop

THEN WHILE value $<=$ stop

DO BEGIN

value := value + step;

WRITE (g, value);

INC(last); 
END

ELSE WHILE value $>=$ stop

DO BEGIN

value : = value +step;

WRITE (g, value);

INC(last);

END;

END;

CLOSE $(\mathrm{g})$;

$\mathrm{DEC}$ (last);

index $:=0$;

value $:=$ start;

set_parameter_to_value;

END;

PROCEDURE exp_data_type.set_parameter_to_value;

BEGIN

CASE key OF

$$
\begin{aligned}
& \text { 'O' : Vo := value; } \\
& \text { 'R' : Vr := value; } \\
& \text { 'W' : lambda := value; } \\
& \text { 'P' : BEGIN } \\
& \text { energy : = value; } \\
& \text { lambda }:=1239.85 / \text { energy; } \\
& \text { END; }
\end{aligned}
$$

'L' : phi := value;

END; $\{$ CASE $\}$

END;

PROCEDURE exp_data_type.open_empty_QE_file;

VAR f : TEXT;

BEGIN

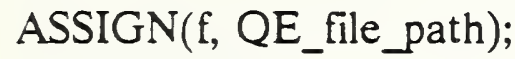

REWRITE(f);

CLOSE(f);

END;

PROCEDURE exp_data_type.write_temp_data;

VAR f : TEXT;

BEGIN

ASSIGN(f, 'temp.dat');

REWRITE(f);

WRITELN(f, model_prm_file_path);

IF key $<>$ 'P'

THEN WRITELN(f, key, ', ', lambda, ', ', phi)

ELSE WRITELN(f, key, ' ', energy, ', ', phi);

WRITELN(f, Vo, ' ', Vr, ' ', shutter);

WRITELN(f, depth, ' ', oxide_t); 
WRITELN(f, QE_file_path);

WRITELN(f, index, ', last);

WRITELN(index, last);

CLOSE(f);

END;

PROCEDURE exp_data_type.update;

VAR dir : DIRSTR;

name : NAMESTR;

ext : EXTSTR;

f : TEXT;

BEGIN

ASSIGN(f, 'temp.dat');

$\operatorname{RESET}(\mathrm{f})$;

READLN(f, model_prm_file_path);

IF key $<>$ 'P'

THEN READLN(f, key, lambda, phi)

ELSE BEGIN

READLN(f, key, energy, phi);

lambda := 1239.85/energy;

END;

READLN(f, Vo, Vr, shutter);

WHILE shutter[1] = ,

DO DELETE(shutter, 1, 1);

$\operatorname{READLN}(f$, depth, oxide_t $)$;

READLN(f, QE_file_path);

READLN(f, index, last);

CLOSE(f);

IF shutter $=$ 'open'

THEN BEGIN

INC(index);

IF key $=$ ' $R$ '

THEN shutter := 'closed';

END

ELSE shutter := 'open';

get_current_value;

set_parameter_to_value;

write_temp_data;

END;

PROCEDURE exp_data_type.get_current_value;

VAR $g$ : file_of_values_type;

BEGIN

$\operatorname{ASSIGN(g,~'temp.val');~}$

$\operatorname{RESET}(\mathrm{g})$;

$\operatorname{SEEK}(\mathrm{g}$, index);

$\operatorname{READ}(\mathrm{g}$, value);

CLOSE $(\mathrm{g})$; 
END;

PROCEDURE exp_data_type.prepare_pq;

CONST eps_SiO2 $=3.9$;

eps_Si $=11.9$;

eps $0=8.8541878 \mathrm{e}-14 ;\{\mathrm{C} /(\mathrm{V} \mathrm{cm})\}$

cm_per_nm $=1 \mathrm{e}-7$;

q_per_C $=1 / 1.60218 \mathrm{e}-19$;

\{and from W. R. Thurber and J. R. Lowney, "Electrical Transport

Properties of Silicon" Chapter 14 in the VLSI Handbook, ed. N.

G. Einspruch (Academic Press, Orlando, 1985), page 188.\}

$E_{c}=30.5 e 4$

$c=0.657$

VAR Nss0, Nss, Rb0, Rb, En0, En, bot, top,

sheet_R_ratio, concentration_ratio : DOUBLE;

\section{BEGIN}

IF shutter $=$ 'closed'

THEN phi : $=0$;

p.s[I_mode] := 'Steady State';

p.s[I_graphs] := 'Specified';

p.i[I_graph_1][0]:= 5;

p.s[I_front_surface] := 'Charged';

p.s[I_spectrum]:= 'Fixed';

p.s[I_light_shape] := 'Fixed';

p.s[I_Vbb_shape] := 'Fixed';

p.r[I_lambda_start][0]:= lambda;

p.r[I_light_start $][0]:=$ phi;

p.r[I_Vbb_start $][0]:=\mathrm{Vr}$;

Nss0 := p.r[I_front_Nss][0];

Nss $:=$ Nss $0+$ q_per_C ${ }^{*}$ Vo $^{*}$ eps_SiO2 $2^{*}$ eps $0 /\left(\right.$ oxide_t $t^{*} \mathrm{~cm} \_$per_nm $)$;

p.r[I_front_Nss][0]:= Nss;

$\mathrm{Rb0}:=$ p.r[I_Rb][0];

IF $((\mathrm{Nss} 0=0)$ OR $(\mathrm{Rb} 0=0))$

THEN BEGIN

$\mathrm{Rb}:=\mathrm{Rb} 0$;

END

ELSE BEGIN

IF Nss $=0$

THEN Nss := Nss + 1e7;

concentration ratio := Nss $/ \mathrm{Nss}$;

En0 := ABS(Nss0/(2*eps_Si*eps0));

En $:=\operatorname{ABS}\left(\mathrm{Nss} /\left(2^{*}\right.\right.$ eps_Si*eps 0$\left.)\right)$;

top $:=1+\operatorname{EXP}\left(c^{*} \operatorname{LN}(\operatorname{En} / \mathrm{Ec})\right) ;$

bot $:=1+\operatorname{EXP}\left(c^{*} \operatorname{LN}(\operatorname{En} 0 / E c)\right)$;

sheet_R_ratio := top/bot;

$\mathrm{Rb}:=\overline{\mathrm{Rb}} 0$ * concentration_ratio*sheet_R_ratio;

WRITELN('conc. ratio $=\overline{-}$, concentration_ratio);

WRITELN('sheet $\mathrm{R}$ ratio = ', sheet_R_ratio); 
END;

p.r[I_Rb] $[0]:=\mathrm{Rb}$;

END;

PROCEDURE exp_data_type.delete_temporary_files;

VAR f : FILE;

dir : DIRSTR;

name : NAMESTR;

ext : EXTSTR;

temp_file_path : PATHSTR;

BEGIN

$\operatorname{ASSIGN(f,~'temp.dat');~}$

$\operatorname{ERASE}(\mathrm{f})$;

FSPLIT(model_prm_file_path, dir, name, ext);

temp_file path $:=$ dir + 'temp.val';

$\operatorname{ASSIGN}(\mathrm{f}$, temp_file_path);

$\operatorname{ERASE}(f)$;

temp_file_path $:=$ dir + 'temp.prm';

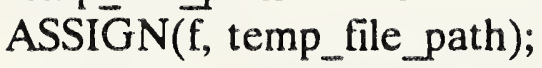

$\operatorname{ERASE}(f)$;

temp_file_path $:=$ dir + 'temp.pdf';

ASSIGNN(f, temp_file_path);

ERASE(f);

END;

PROCEDURE prm_data_type.read_prm_file;

VAR f : FILE;

count, result : WORD;

BEGIN

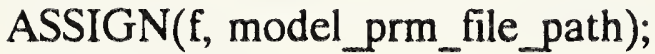

$\operatorname{RESET}(\mathrm{f}, 1)$;

count $:=16^{*} \mathrm{SUCC}\left(\mathrm{ORD}\left(\mathrm{I} \_\mathrm{Nc}\right)\right)$;

BLOCKREAD(f, exp_data.p.s, count, result);

count $:=16^{*} 3^{*} \mathrm{SUCC}\left(\mathrm{ORD}\left(\mathrm{I}_{\text {_Abs_Tg }}\right)\right.$ );

BLOCKREAD(f, exp_data.q.s, count, result);

CLOSE(f);

END;

PROCEDURE prm_data_type.write_prm_file;

VAR f : FILE;

dir : DIRSTR;

name : NAMESTR;

ext : EXTSTR;

temp_prm_file_path : PATHSTR;

count, result : WORD;

BEGIN

FSPLIT(model_prm_file_path, dir, name, ext);

temp_prm_file_path $:=$ dir + 'temp.prm'; 


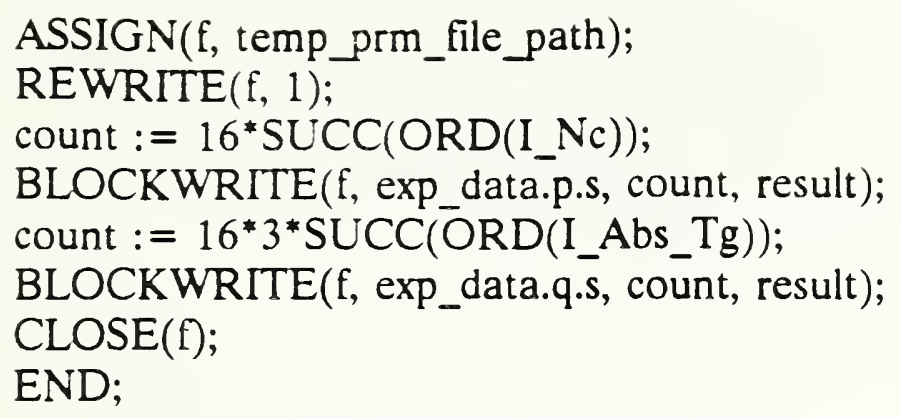

PROCEDURE test_exp_data;

\section{BEGIN}

WITH exp_data

DO BEGIN

WRITELN(model_prm_file_path);

WRITELN(p.s[I_mode]);

WRITELN(p.s[I_graphs]);

WRITELN('Graph number = ', p.i[I_graph_1][0]);

WRITELN(p.s[I_spectrum]);

WRITELN(p.s[I_light_shape]);

WRITELN('lambda =', p.r[I_lambda_start][0]);

WRITELN('phi = ', p.r[I_light_start][0]);

WRITELN('Vo = ', Vo);

WRITELN('Nss = ', p.r[I_front_Nss][0]);

WRITELN('Vr = ', p.r[I_Vbb_start][0]);

END;

END;

BEGIN \{main

WITH exp_data

DO BEGIN

WRITELN('MAKE_PRM.EXE is initializing or updating as appropriate.');

initialize_or_update;

WRITEL'N('The shutter is now', shutter);

IF ( index $>=$ last)

THEN BEGIN

WRITELN('MAKE_PRM.EXE is deleting the temporary files.');

delete_temporary_files;

END

ELSE BEGIN

WRITELN('MAKE_PRM.EXE is reading', model_prm_file_path);

read_prm_file;

\{test_exp_data;

WRITELN('MAKE_PRM.EXE is preparing the temporary parameter file.');

prepare_pq;

\{test_exp_data;\}

WRITELN('MAKE_PRM.EXE is writing TEMP.PRM.');

write_prm_file; 
WRITELN;

END;

END;

END. 
APPENDIX II.AListing of READ_PDF.PAS that compiles under Turbo Pascal Version 5.5.

PROGRAM read_total_photocurrent_from_pdf_file;

$\{\$ N+\}$

USES CRT, DOS;

TYPE pdf_data_type

$=$ OBJECT

model_prm_file_path, QE_file_path : PATHSTR;

key : $\overline{\mathrm{C}} \mathrm{HA} \overline{\mathrm{R}}$;

shutter : STRING[7];

lambda, energy, phi, Vo, $\mathrm{Vr}$,

oxide tt, depth, J0, Jt, QE : DOUBLE;

$z:$ ARRAY[0..500] OF STRING[30];

PROCEDURE initialize;

PROCEDURE read_pdf_file;

PROCEDURE write_J0_to_file;

PROCEDURE read_J0_from_file;

PROCEDURE append_- $\bar{Q} E$ _file;

END;

VAR pdf_data : pdf_data_type;

PROCEDURE pdf_data_type.initialize;

VAR f : TEXT;

BEGIN

ASSIGN(f, 'temp.dat');

$\operatorname{RESET}(\mathfrak{f})$;

READLN(f, model_prm_file_path);

READLN(f, key, lambda, phi);

IF key $=$ ' $\mathrm{P}$ '

THEN BEGIN

energy := lambda;

lambda := 1239.85/energy;

END;

$\operatorname{READLN}(\mathrm{f}, \mathrm{Vo}, \mathrm{Vr}$, shutter);

WHILE shutter[1] = ,

DO DELETE(shutter, 1, 1);

$\operatorname{READLN}(\mathfrak{f}$, depth, oxide_t $)$;

READLN(f, QE_file_path);

CLOSE(f);

END;

PROCEDURE pdf_data_type.write_J0_to_file;

VAR f : TEXT;

BEGIN 
$\{\$ I-\}$

ASSIGN(f, 'J0.dat');

$\operatorname{RESET}(\mathrm{f})$;

CLOSE $(\mathrm{f})$;

$\{\$ I+\}$

ASSIGN(f, 'J0.dat');

IF $($ IORESULT $=0$ )

THEN APPEND(f)

ELSE REWRITE(f);

WRITELN(f, J0);

CLOSE(f);

END;

PROCEDURE pdf_data_type.read_J0_from_file;

VAR f : TEXT;

BEGIN

ASSIGN(f, 'J0.dat');

$\operatorname{RESET}(\mathfrak{f})$;

WHILE NOT EOF $(f)$

DO READLN(f, J0);

CLOSE(f);

END;

PROCEDURE pdf_data_type.read_pdf_file;

CONST m_per_um = 1e-6;

VAR f : TEXT;

line : STRING[80];

n, check, how_many : INTEGER;

$\mathrm{x}$ : DOUBLE;

pdf file path : PATHSTR;

dir : DIRSTR;

name : NAMESTR;

ext : EXTSTR;

BEGIN

FSPLIT(model_prm_file_path, dir, name, ext);

pdf_file_path := dir + 'temp.pdf';

$\operatorname{ASSIGN(f,pdf\_ file\_ path);~}$

$\operatorname{RESET}(\mathrm{f})$;

FOR $n:=1$ TO 8

DO READLN(f, line);

READLN(f, how many);

FOR $n:=1$ TO how_many

DO READLN(f, line);

READLN(f, check);

FOR $\mathrm{n}:=1$ TO how many

DO READLN(f, line);

READLN(f, check);

$\mathrm{x}:=-1$ 
WHILE $\mathrm{x}<$ depth $^{*} \mathrm{~m}_{\text {_per_um }}$

DO IF $V_{r}=0$

THEN BEGIN

$$
\mathrm{J} 0:=0
$$

$\operatorname{READLN}(\mathrm{f}, \mathrm{x}, \mathrm{Jt})$;

END

ELSE IF shutter $=$ 'closed'

THEN READLN $(f, x, J 0)$

ELSE READLN(f, $x, J t)$;

CLOSE(f);

IF $\mathrm{Vr}<>0$

THEN IF shutter $=$ 'closed'

THEN write_J0_to_file

END;

ELSE read_Jo_from_file;

PROCEDURE pdf_data_type.append_QE_file;

CONST lambda_0 $=1239.85$; $\{$ Lambda in $\mathrm{nm}$ at which $1 \mathrm{~W}$ gives $1 \mathrm{~A}$ at $100 \% \mathrm{QE}$.

VAR f : TEXT;

Jnet, J100 : DOUBLE;

BEGIN

Jnet := ABS(Jt-J0);

$\mathrm{J} 100:=$ lambda ${ }^{*}$ phi/lambda_0;

$\mathrm{QE}:=\mathrm{Jnet} / \mathrm{J} 100$;

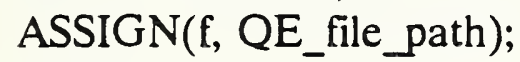

APPEND(f);

CASE key OF

'O' : WRITELN(f, Vo:9:6, ' ', QE:9:6);

'R' : WRITELN(f, Vr:9:6, ' ', QE:9:6);

'W' : WRITELN(f, lambda:9:3, ' ', QE:9:6);

'P' : WRITELN(f, energy:9:6, ' , QE:9:6);

'L' : WRITELN(f, phi:12, ', QE:9:6);

END; $\{$ CASE $\}$

CLOSE(f);

END;

\section{BEGIN \{main}

WITH pdf_data

DO BEGIN

initialize;

WRITELN('READ_PDF.EXE is reading TEMP.PDF.');

read_pdf_file;

IF shutter $=$ 'open'

THEN BEGIN

WRITELN('READ_PDF.EXE is writing ', QE_file_path);

append_QE_file;

END; 
END;

END. 
APPENDIX III. A sample of the screen produced by MAKE_PRM.EXE when RUN_PCID.BAT is executed.

Parameter file (including PRM extension) defining photodiode:

1337_new.prm

Complete path to file in which to store QE results:

test.dat

Type of experiment to simulate?

(O)xide bias (Volts) scan?

(R)everse bias (Volts) scan?

(W)avelength $(\mathrm{nm})$ scan?

(P)hoton energy $(\mathrm{eV})$ scan?

(L)inearity $\left(\mathrm{W} / \mathrm{cm}^{\wedge} 2\right)$ scan?

Enter letter here: $w$

Start, stop, and stop values from (K)eyboard or all values from (F)ile? $\mathrm{k}$

First value for scanned parameter: 400

Stop value for scanned parameter: 900

Step size for scanned parameter: 10

Incident irradiance (W per sq. $\mathrm{cm}$ ): $1 \mathrm{e}-5$

Oxide bias voltage (V): 0

Reverse bias voltage (V): 0

Non-zero thickness of front surface oxide $(\mathrm{nm}): 25$

Depth at which to get total photocurrent (um): 100 


\title{
Part II: INTERPRETING OXIDE-BIAS EXPERIMENTS
}

\author{
Jon Geist \\ NIST, Gaithersburg, MD 20899 \\ Rainer Köhler and Roland Goebel \\ BIPM, F-92310 Sevre, France \\ A. M. Robinson and C. R. James \\ University of Alberta, Alberta, Canada T6G 2G7
}

\begin{abstract}
The semiconductor device modeling program PC-1D and the programs that support its use in high-accuracy modeling of photodiodes, all of which were described in Part I of this paper, are used to simulate oxide-bias self-calibration experiments on three different types of silicon photodiodes. It is shown that these simulations can be used to determine photodiode characteristics, including the internal quantum efficiency for the different types of photodiodes. In the latter case, the simulations provide more accurate values than can be determined by using the conventional data reduction procedure, and an uncertainty estimate can be derived. Finally, it is shown that $0.9997 \pm 0.0003$ is a nominal value for the internal quantum efficiency of one type of photodiode over the 440 to $460 \mathrm{~nm}$ spectral region.
\end{abstract}

Key words: induced junction photodiode; inversion layer photodiode; numerical modeling; numerical simulation; oxide-bias experiment; quantum efficiency

\section{Introduction}

Part II presents the results of high-accuracy simulations of various oxide-bias experiments performed on silicon photodiodes. The simulations were carried out by using the semiconductor device modeling program PC-1D and a set of three programs designed to support the use of PC-1D in this specific application. Both PC-1D and the support programs are described in Part I.

The oxide-bias experiment [1-3] consists of recording the ratio of the photocurrent as a function of oxide-bias voltage to the zero-bias photocurrent during irradiation of the photodiode with a stable photon flux. The oxide bias is usually applied with a transparent water-drop electrode. A review is given in Ref. [3].

Part II is organized as follows: Section 2 describes the simulation of an oxide-bias, selfcalibration experiment on an EG\&G UV444B type photodiode. The purpose of this simulation is to demonstrate the derivation of an accurate value for the zero-bias quantum efficiency including an uncertainty estimate. Section 3 describes the simulation of an oxidebias experiment on a Hamamatsu 1337 type photodiode. The purpose of this simulation is to determine nominal values for the charge trapped in the oxide covering the front surface of the photodiode, and for the surface recombination velocity at the oxide-silicon interface. Section 4 describes the simulation of an oxide-bias experiment on a UDT UV100 type photodiode. The purpose of this simulation is to determine a nominal high-accuracy value 
for the internal quantum efficiency of this type of photodiode and the nominal wavelength region where this value is valid. Section 5 gives the conclusions of $P$ art II.

\section{EG\&G UV444B Photodiode}

The EG\&G UV444B silicon photodiode was the first of its type to be self-calibrated by using the oxide-bias experiment [2]. This type of photodiode consists of a $300 \mu \mathrm{m}$ thick, 300 $\Omega \mathrm{cm}$, phosphorus-doped, n-type silicon substrate with an $\mathrm{n}^{+}$-type phosphorus diffusion in the rear surface, a $\mathrm{p}^{+}$-type boron diffusion in the front surface, and a nominally $120 \mathrm{~nm}$ thick, thermally grown oxide-passivation layer over the front surface. Figure 1 shows the front-region doping profile that was obtained from spreading resistance measurements on a representative UV444B photodiode. Figure 1 also shows the equilibrium hole concentration calculated for this doping distribution by using PC-1D.

The rear-region, $\mathrm{n}^{+}$-type diffusion in the UV444B was not modeled; instead it was assumed that the recombination velocity at the rear of the device was zero. This assumption is not physically realizable, but it results in a mirror for minority carriers at the rear of the device, just as does the $\mathrm{n}^{+}$-type diffusion. This assumption is useful because it requires fewer finite elements, thereby leaving more of them available for modeling the front region and depletion region.

Figure 2 compares simulated and experimental results of an oxide-bias experiment on a UV444B photodiode for irradiation by a $3 \mathrm{~mm}$ diameter spot of $476.2-\mathrm{nm}$ laser radiation through a water-drop electrode. Reference [4] describes the apparatus used to obtain the experimental data. The absorption-coefficient data in the file SIL_WEAK.ABS used for this simulation were calculated from an equation [5] that was fit to the data measured by Weakliem and Redfield [6]. These data are described in Part I. Both the surface recombination velocity $S$ and the number density $N_{\text {, }}$ of the oxide fixed charge were adjusted in the simulation to obtain a good fit to the experimental data in figure 2.

The procedure used to fit the simulated data to the experimental data shown in figure 2 was as follows: first, a value of $S$ was chosen, then the value of $N_{8}$, was adjusted until the average residual of the fit to the photocurrent ratio was zero for the last two data points in the figure. This gave a value of $\mathrm{N}_{\text {.. }}$ for each value of $\mathrm{S}$ that was chosen. The best combination of $\mathrm{S}$ and $\mathrm{N}_{\text {. }}$ was then obtained by minimizing the residual standard deviation over all of the data shown in figure 2.

Figures 3, 4, and 5 plot the residuals (differences) between the results of the fits and the experimental results and table 1 lists the residual standard deviation for three different choices of $S$ and $N_{.}$. It is clear by inspection of the figures that the combination of $S$ and

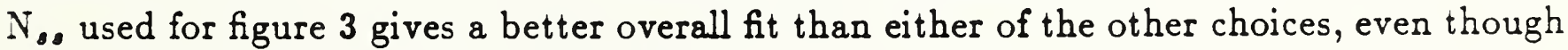
the residual standard deviation for figure 3 is not very much lower than that for figure 4 or figure 5 . One reason for the relatively small decrease in residual standard deviation with the best fit is the effect of the apparent outlier points near $-1 \mathrm{~V}$ and $-10.5 \mathrm{~V}$ oxide bias.

The assignment of $\mathrm{S}=6000 \mathrm{~cm} / \mathrm{s}$ and $\mathrm{N}_{.,}=1.942 \times 10^{12} \mathrm{~cm}^{-2}$ with estimated uncertainties of $\pm 1000 \mathrm{~cm} / \mathrm{s}$ and $\pm 0.070 \times 10^{12} \mathrm{~cm}^{-2}$, respectively, is justified by figures 3,4 , and 5 . This 


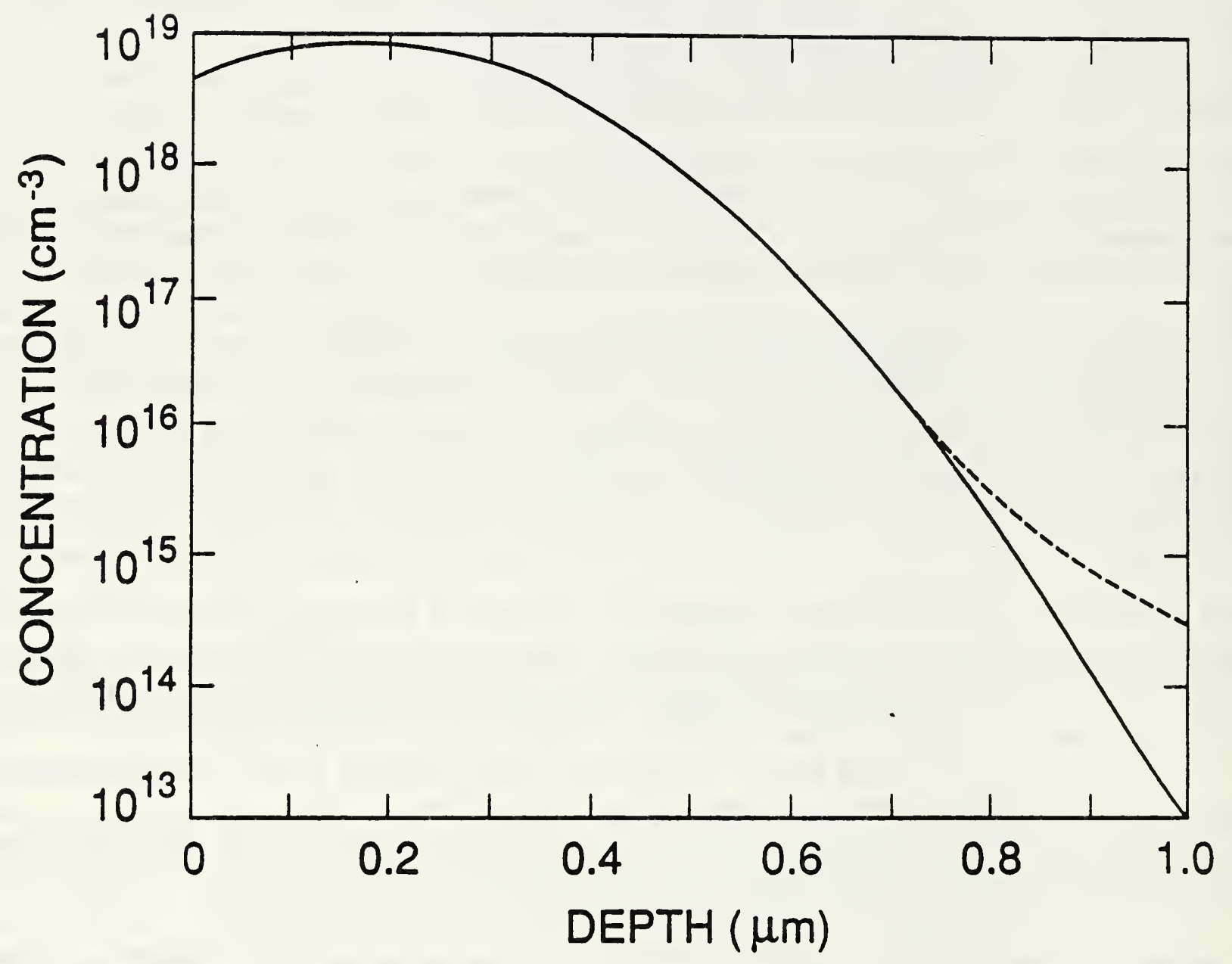

Figure 1. The front-region boron concentration (continuous line) and the equilibrium hole concentration (dashed line) calculated by PC-1D for a typical EG\&G UV444B type photodiode. 


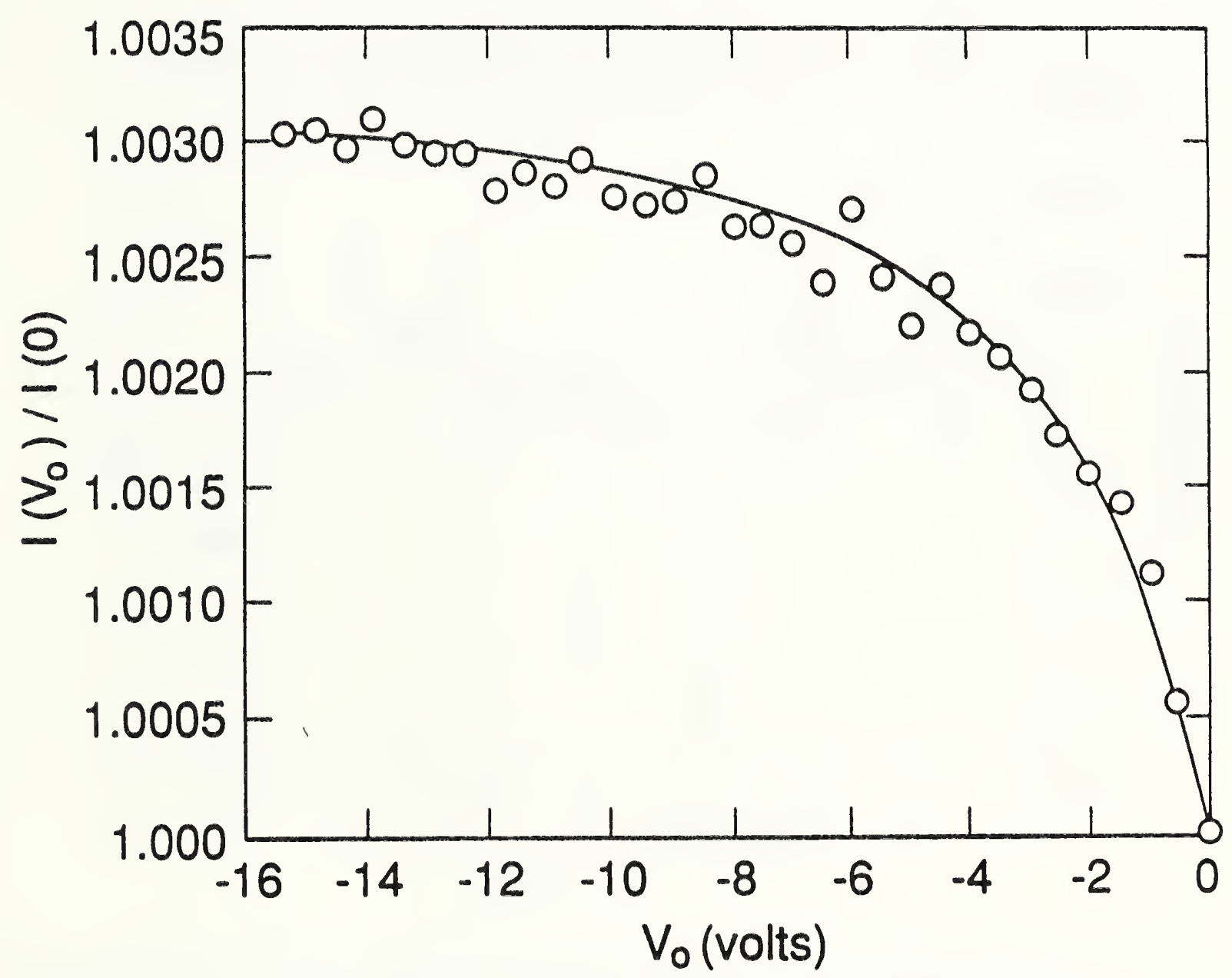

Figure 2. Ratio of the photocurrent $I\left(V_{0}\right)$ as a function of oxide-bias voltage $V_{0}$ to the photocurrent $\mathrm{I}(0)$ at zero-bias voltage as measured on an EG\&G UV444B silicon photodiode and as calculated with PC-1D with a front-surface recombination velocity $S=6000$ $\mathrm{cm} / \mathrm{s}$ and an oxide trapped charge number density $\mathrm{N}_{\varrho}=1.942 \times 10^{12} \mathrm{~cm}^{-2}$. 


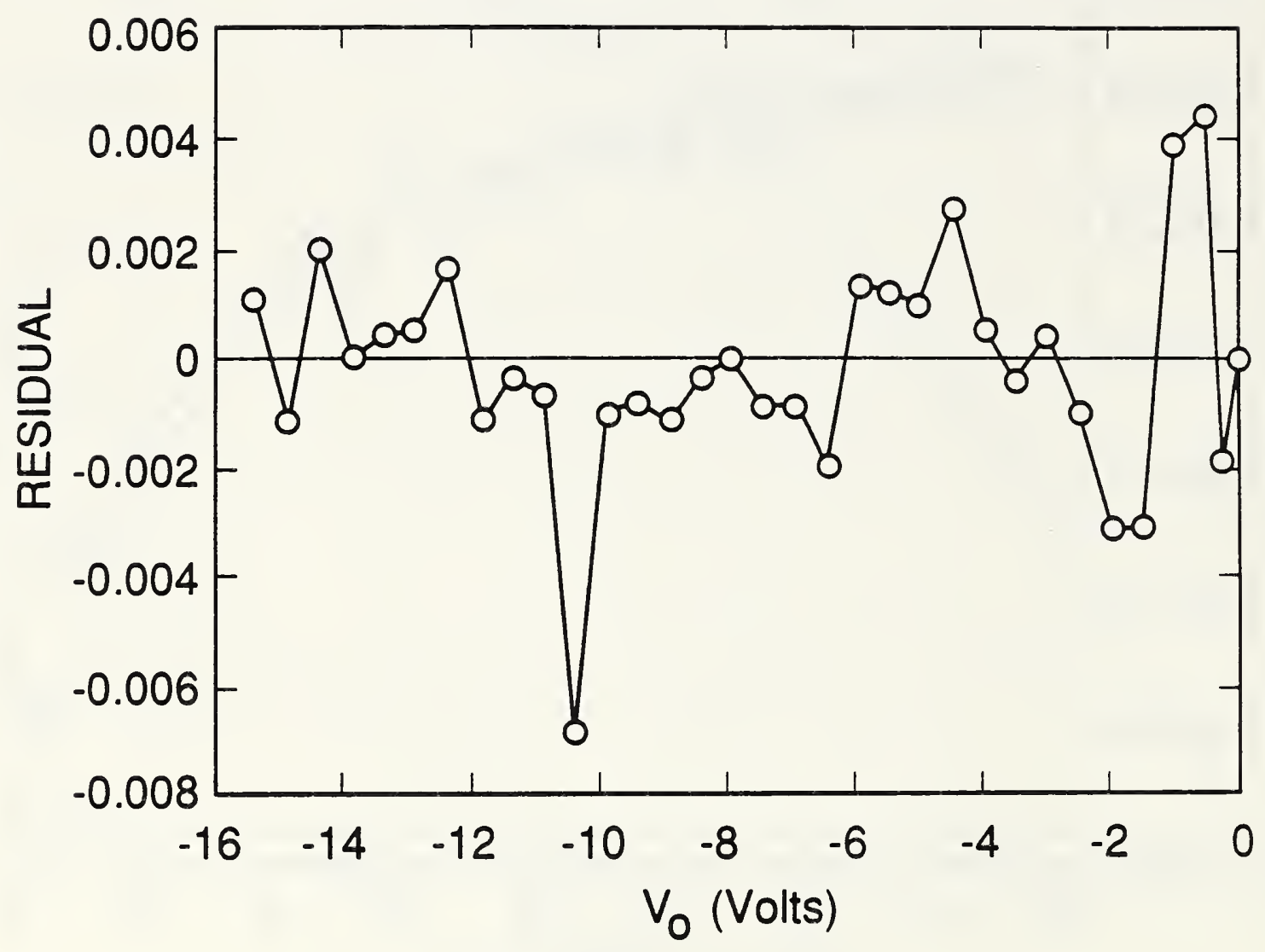

Figure 3. The residuals between the experimental data of figure 2 and the simulated data calculated by PC-1D when $\mathrm{S}=6000 \mathrm{~cm} / \mathrm{s}$ and $\mathrm{N}_{\varrho,}=1.942 \times 10^{12} \mathrm{~cm}^{-2}$. 


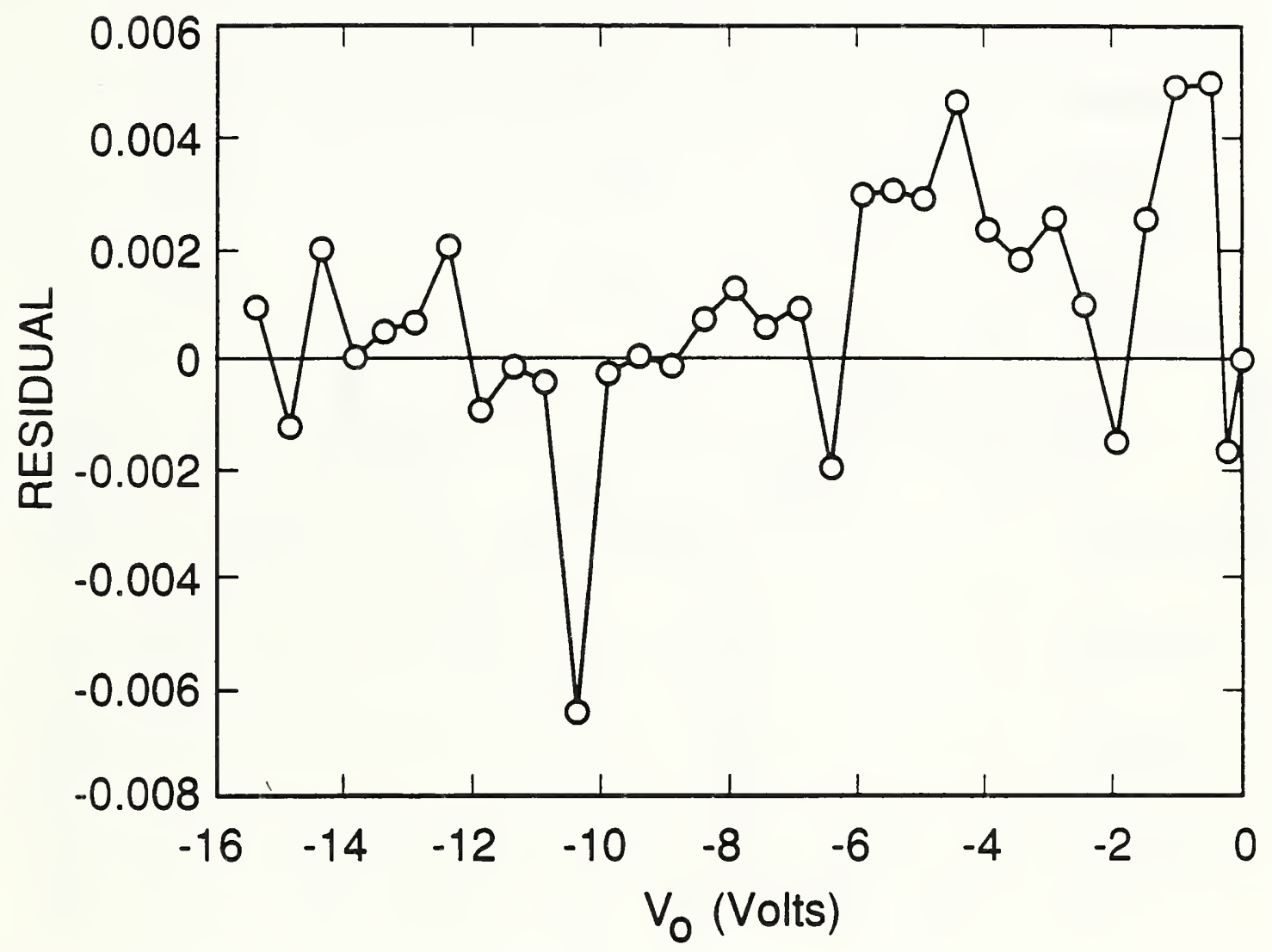

Figure 4. The residuals between the experimental data of figure 2 and the simulated data calculated by PC-1D when $\mathrm{S}=5000 \mathrm{~cm} / \mathrm{s}$ and $\mathrm{N}_{\theta .}=2.011 \times 10^{12} \mathrm{~cm}^{-2}$. 


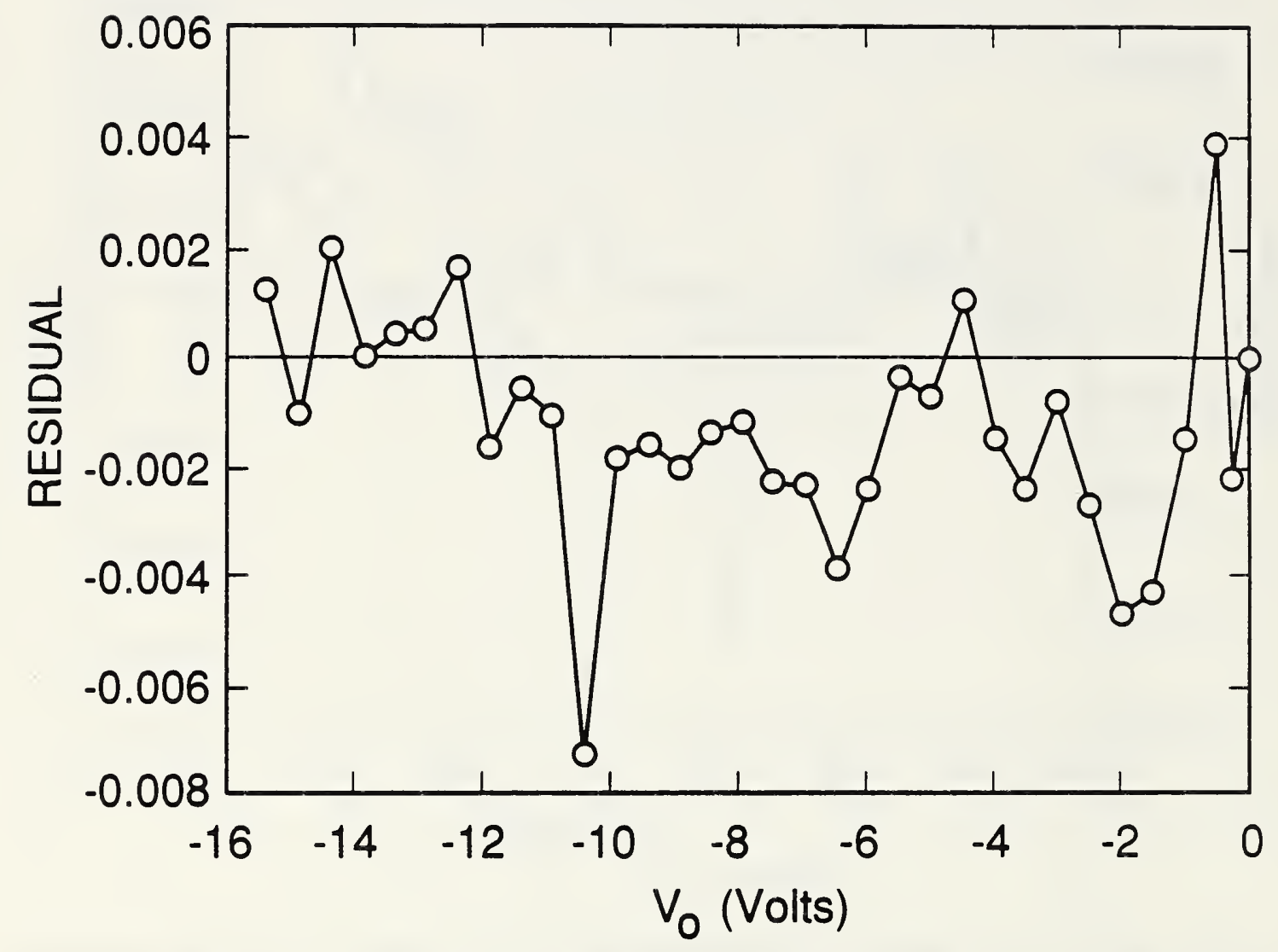

Figure 5. The residuals between the experimental data of figure 2 and the simulated data calculated by PC-1D when $S=7000 \mathrm{~cm} / \mathrm{s}$ and $N_{, \prime}=1.883 \times 10^{12} \mathrm{~cm}^{-2}$. 
Table 1. Comparison of simulated oxide bias results with experimental results for a UV444B type photodiode where Res. Std. Dev. is the standard deviation of the difference between simulated and experimental results.

$\begin{array}{ccc}\mathrm{S}(\mathrm{cm} / \mathrm{s}) & \mathrm{N}_{\text {ss }}\left(10^{12} \mathrm{~cm}^{-3}\right) & \text { Res. Std. Dev. } \\ 5000 & 2.011 & 0.002233 \\ 6000 & 1.942 & 0.002076 \\ 7000 & 1.883 & 0.002093\end{array}$


assignment corresponds to an internal quantum deficiency (one minus the internal quantum efficiency) at zero oxide bias of $0.1690 \pm 0.0021$. The stated uncertainty is the maximum difference between the internal quantum deficiency calculated for the combination of $S$ and $\mathrm{N}_{s}$ used in figure 3 , and those calculated for the combinations of $\mathrm{S}$ and $\mathrm{N}_{\boldsymbol{s}}$ used for figures 4 and 5 .

The assumption that the average of the last two data points in figure 3 corresponds to $100 \%$ internal quantum efficiency gives a zero-bias internal quantum deficiency of 0.1634 with no reliable way to estimate the uncertainty associated with that value. This assumption, which is usually used with the oxide-bias, self-calibration experiment, produces a result that differs from the more accurate value determined from the fit by 0.0056 . This difference is about $3.3 \%$ of the effect being calculated, and more than 2.5 times the uncertainty associated with the more accurate value.

It must be emphasized that the results reported here are illustrative rather than definitive since the doping distribution data in figure 1 were obtained from one photodiode, and the oxide-bias data in figure 2 were obtained from a different photodiode. An additional uncertainty associated with the effect of variations in doping distribution would be needed, or the doping distribution must be measured on the actual photodiode used in the selfcalibration.

Because oxide bias voltages large enough to nearly saturate the photodiode response are also large enough to change $\mathrm{S}$ or $\mathrm{N}_{\Delta, \Delta}$, the oxide-bias measurement is already destructive to a certain extent, and it must be carried out as described in Ref. [7] in order to yield the highest accuracy of which it is capable. Therefore, the sacrifice of the photodiode for a spreading resistance measurement is not as impractical as it might at first seem. Nevertheless, it is inconvenient, and more convenient approaches are being developed $[3$.

The results reported here might be considered illustrative for another reason. The fitting and uncertainty analysis were carried out by hand in a somewhat subjective manner. Ideally, the fitting and uncertainty analysis would be carried out automatically by a fitting routine incorporated into the set of programs used to run PC-1D for photodiode modeling. However, this task might not be as straightforward as it first would seem because the fit should be constrained to pass through the mean of the last few data points at the highest oxide bias voltage, as it was in the hand fit reported here. Without this constraint, the minimum residual standard deviation might be obtained for a choice of parameters that clearly does not fit the data well at the highest oxide bias voltages. It is clear that the data at the highest oxide bias voltages should be weighted more heavily in the fit, but it is not clear how much more heavily. Therefore, an automated program does not eliminate the subjectivity, but only transfers it to the weighting of the data.

\section{Hamamatsu 1337 Photodiode}

Like the EG\&G UV444B photodiode, the Hamamatsu 1337 is a $\mathrm{p}^{+} \mathrm{nn}^{+}$photodiode, but its front-region doping profile is shallower, its background n-type dopant concentration is $5 \times 10^{12} \mathrm{~cm}^{-3}$, and its oxide-passivation layer has a nominal thickness of $25 \mathrm{~nm}$. Figure 6 


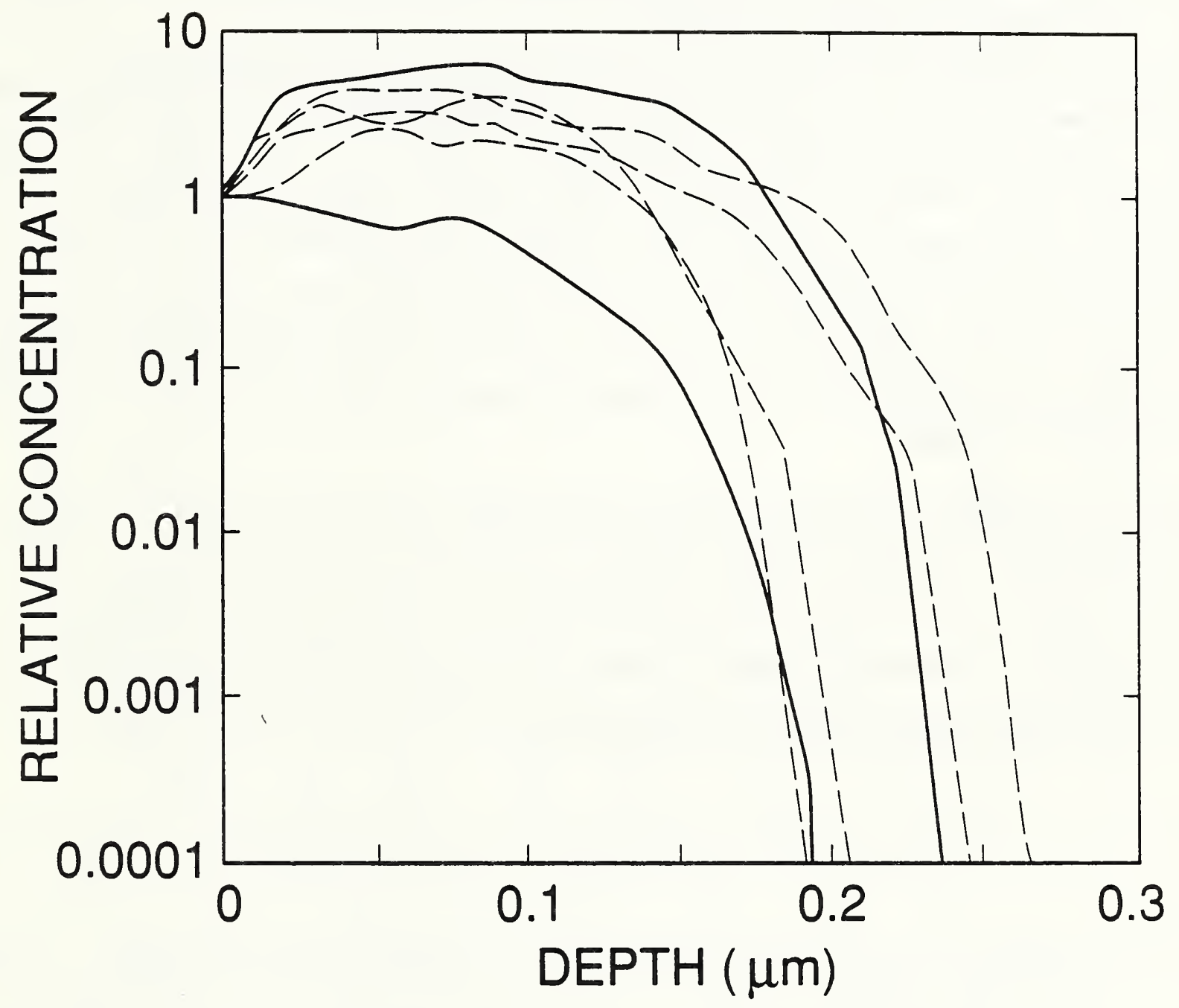

Figure 6. The relative front-region boron concentration for six typical Hamamatsu 1337 type photodiodes. The two profiles shown with solid lines bracket the other four in their effect on the shape of the internal quantum-deficiency spectrum. 
shows relative front-region doping profiles for six different Hamamatsu 1337 photodiodes taken from four different batches. The curves in figure 6 were normalized to unity at the oxide-silicon interface because it is the shape of the doping profile rather than the magnitude that has the major effect on the internal quantum efficiency [8]. The measured dopant concentrations at the oxide-silicon interface varied between $2 \times 10^{18}$ and $1 \times 10^{19}$ $\mathrm{cm}^{-3}$ for the six photodiodes of figure 6 .

Figure 7 shows the results of modeling an oxide-bias experiment on a 1337 type photodiode at $476.2 \mathrm{~nm}$ by using the doping profile that corresponds to the lower solid curve in figure 6. The fit shown in figure 7 was obtained with $\mathrm{N}_{\varrho 0}=-3.0 \times 10^{12} \mathrm{~cm}^{-2}$ and $\mathrm{S}=$ $1.835 \times 10^{5} \mathrm{~cm} / \mathrm{s}$, and gave a zero-bias internal quantum deficiency of 0.003199 . A fit that looked virtually identical to that shown in figure 7 was obtained for the doping profile corresponding to the upper solid curve in figure 6 by using $\mathrm{N}_{2,}=-4 \times 10^{12} \mathrm{~cm}^{-2}$ and $\mathrm{S}$ $=1.56 \times 10^{5} \mathrm{~cm} / \mathrm{s}$. This fit gave a zero-bias internal quantum efficiency of 0.003280 . As in the case of the UV444B type photodiode, the SIL_WEAK.ABS absorption-coefficient data were used, and the rear of the photodiode was modeled as uniformly doped with zero recombination velocity.

Figure 8 compares the internal quantum deficiencies calculated from the doping profile corresponding to the solid curves in figure 6 by using the values of $N_{s}$ and $S$ that were obtained from the simulated oxide-bias experiments based on each of those profiles. The fact that the two curves have very similar shapes is very important. It suggests that a single function of wavelength might be used to extrapolate the internal quantum efficiency of any 1337 type photodiode, rather than a different function for each different photodiode. This possibility is examined in detail in Part III.

Notice that the sign of the trapped charge in the 1337 type photodiode is the opposite of that for the UV444B photodiode, and that the interface recombination velocity is much larger. Both the sign of the trapped charge and the large value of the recombination velocity are consistent with an oxide prepared by chemical vapor deposition rather than by thermal oxidation, because the former is known to produce trapped positive charge and a low surface recombination velocity. The modeling carried out here shows that a large negative charge in the oxide more than makes up for the greater surface recombination velocity by repelling minority carriers from the surface.

The fact that the 1337 type photodiode has negative rather than positive charge trapped in its oxide is important for its possible use in quantum-efficiency interpolation and extrapolation. If the oxide contained a significant amount of positive charge, as assumed in Ref. [9], the oxide-silicon interface would be depleted of holes, leading to a very large decrease in the energy needed to impact-ionize a valence band electron. However, since the oxide contains negative charge, the interface is accumulated. In this case, even though the charge density is quite large, there is little or no decrease in the energy needed to impact-ionize a valence band electron [9]. This is important because a large decrease in the energy required to impact-ionize a valence band electron might cause the quantum yield [10] for electron-hole pair production of the silicon near the front surface to exceed unity at wavelengths as long as $450 \mathrm{~nm}$. This would limit the usefulness of this type 


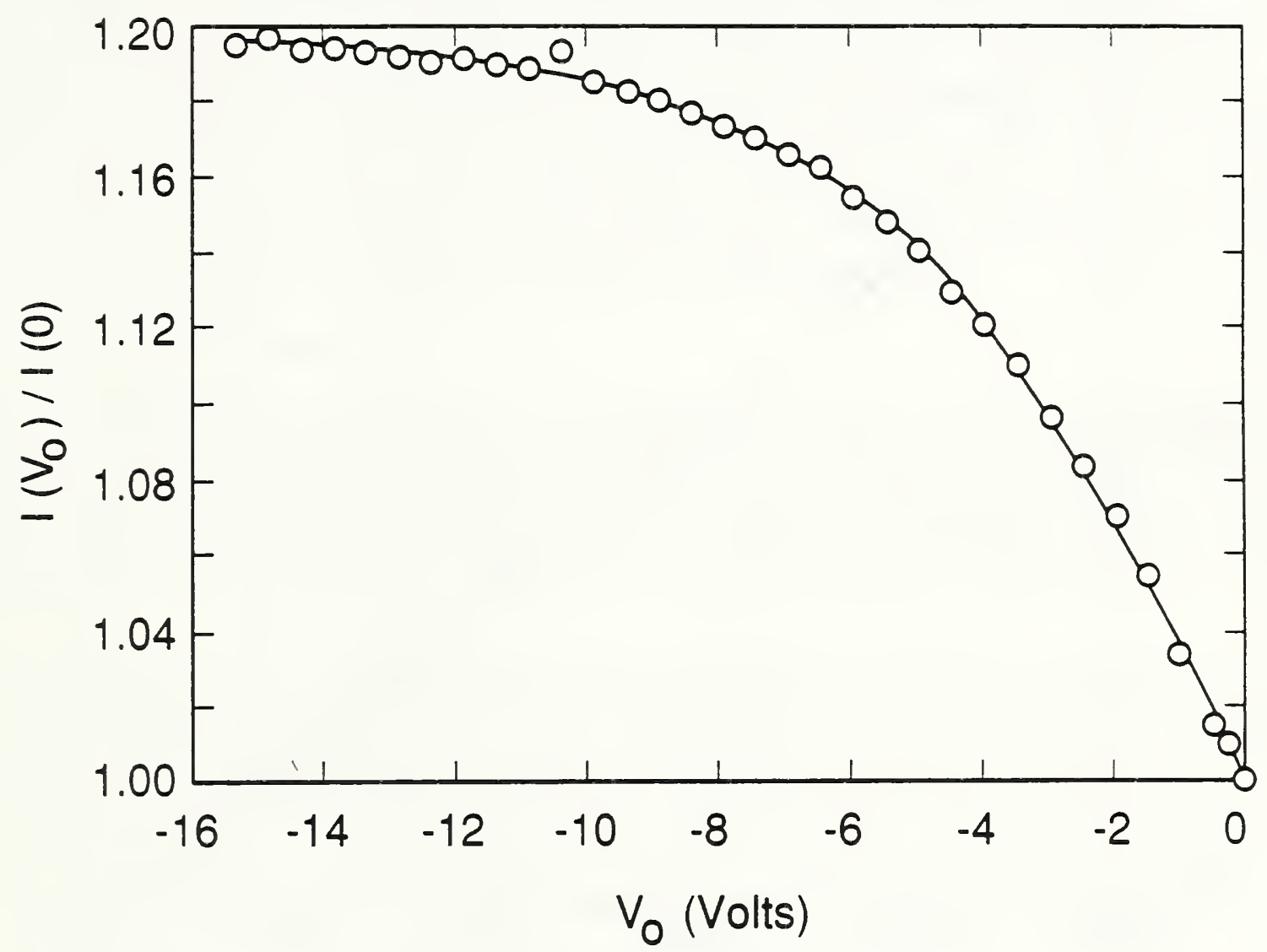

Figure 7. Ratio of the photocurrent $I\left(V_{0}\right)$ as a function of oxide-bias voltage $V_{0}$ to the photocurrent $\mathrm{I}(0)$ at zero-bias voltage as measured on a Hamamatsu 1337 silicon photodiode and as calculated with PC-1D for the doping distribution corresponding to the upper solid curve in figure 6 with $\mathrm{S}=1.835 \mathrm{~cm} / \mathrm{s}$ and $\mathrm{N}_{\Theta}=-3.0 \times 10^{12} \mathrm{~cm}^{-2}$. 


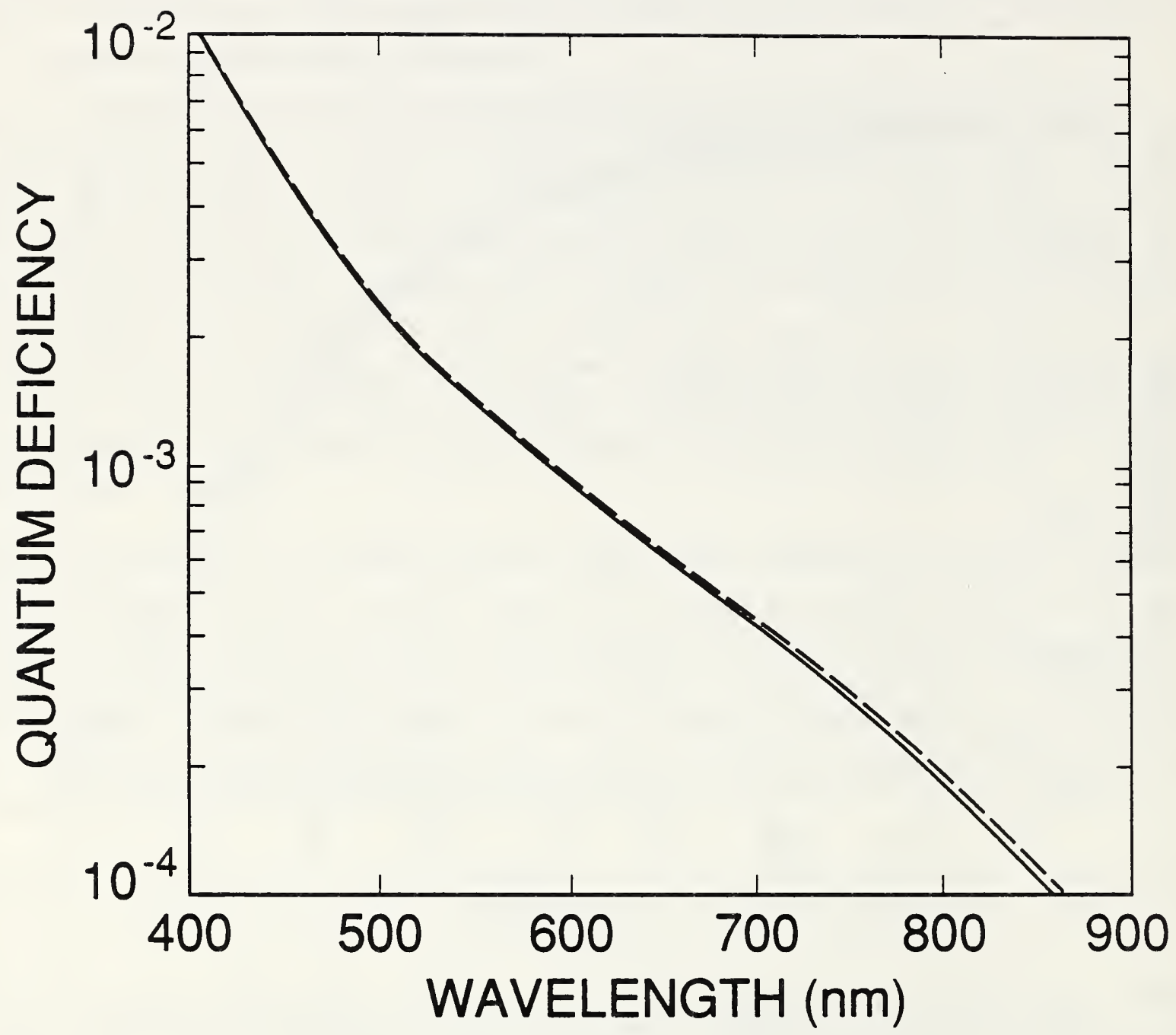

Figure 8. Simulated internal quantum deficiencies for the 1337 type photodiode having the oxide-bias data shown in figure 7: (solid curve) for the doping profile that corresponds to the lower solid curve in figure 6 with $\mathrm{N}_{9 \prime}=-3.0 \times 10^{12}$ and $\mathrm{S}=1.835 \times 10^{5} \mathrm{~cm} / \mathrm{s}$, and (dashed curve) for the doping profile that corresponds to the upper solid curve in figure 6 with $\mathrm{N}_{\text {, }}=-4 \times 10^{12}$ and $\mathrm{S}=1.56 \times 10^{5} \mathrm{~cm} / \mathrm{s}$. 
of photodiode for extrapolating and interpolating quantum-efficiency calibrations. If this limitation were encountered, it could only be overcome with a very accurate model of the quantum yield in the presence of surface fields. Such a model appears well beyond the current state of the art [9].

Even though the uncertainties in the internal quantum deficiency associated with the diode-to-diode variations in doping profile are quite small for the 1337 type photodiode, the oxide-bias experiment is still less than ideal for self-calibration [3]. However, since the 1337 is so well suited for interpolating and extrapolating internal quantum-deficiency data, as shown in Part III, all that is needed are ways to calibrate the photodiode that work over limited spectral regions near $450 \mathrm{~nm}$ and near $900 \mathrm{~nm}$. The next section illustrates how simulations can be used to predict the internal quantum efficiency of a different type of photodiode with an uncertainty of about 0.0003 at $450 \mathrm{~nm}$.

\section{UDT UV100 Photodiode}

The UDT UV100 type photodiode is usually assumed to have an internal quantum effciency of unity in the short wavelength portion of the visible [11]. In fact, this assumption is the basis for the use of the QED 100 and QED 200 multiple reflection radiometers [12], which contain modified UV100 photodiodes, as high-accuracy absolute radiometric standards.

As the state of the art in absolute radiometry improves with time, the assumption of unity quantum efficiency for the UV100 type photodiode becomes more questionable. What is needed is a nominal internal quantum-deficiency spectrum accompanied by a reliable uncertainty spectrum. Since the 1337 type photodiode can be used to interpolate or extrapolate internal quantum-efficiency calibrations, as shown in Part III, it is only necessary to have these spectra over a limited spectral region above $400 \mathrm{~nm}$. (Below $400 \mathrm{~nm}$, the non-unity quantum yield would be a problem.) One way to obtain these spectra is to calculate them from nominal characteristics of the UV100 type of photodiode. The pertinent characteristics can be obtained by fitting numerical simulations of oxide-bias measurements to experimental results as demonstrated in the preceding sections.

The UV100 type of photodiode is quite different from the UV444B and 1337 type photodiodes. Its $\mathrm{n}^{+} \mathrm{p}$ junction is not formed by introducing a front-region dopant, but is induced in a $100 \Omega \mathrm{cm}$ p-type (approximately $1.3 \times 10^{14} \mathrm{~cm}^{-3}$, boron-doped) silicon substrate by the growth of an oxide passivation layer having a large concentration of trapped positive charge [13].

Figure 9 compares the results of simulated and experimental oxide-bias experiments on a UV100 type photodiode. Here again the absorption-coefficient data in SIL_WEAK.ABS were used, and the rear of the photodiode was modeled as uniformly doped with a surface recombination velocity of zero. Since the UV100 type photodiode is an $\mathrm{n}^{+} \mathrm{p}$ type rather than a $\mathrm{p}^{+} \mathrm{n}$ type, the effect of increasing negative bias is to decrease rather than increase the photocurrent. However, because the zero-bias quantum efficiency is so close to unity, this procedure samples the portion of the curve with the most information about the values 


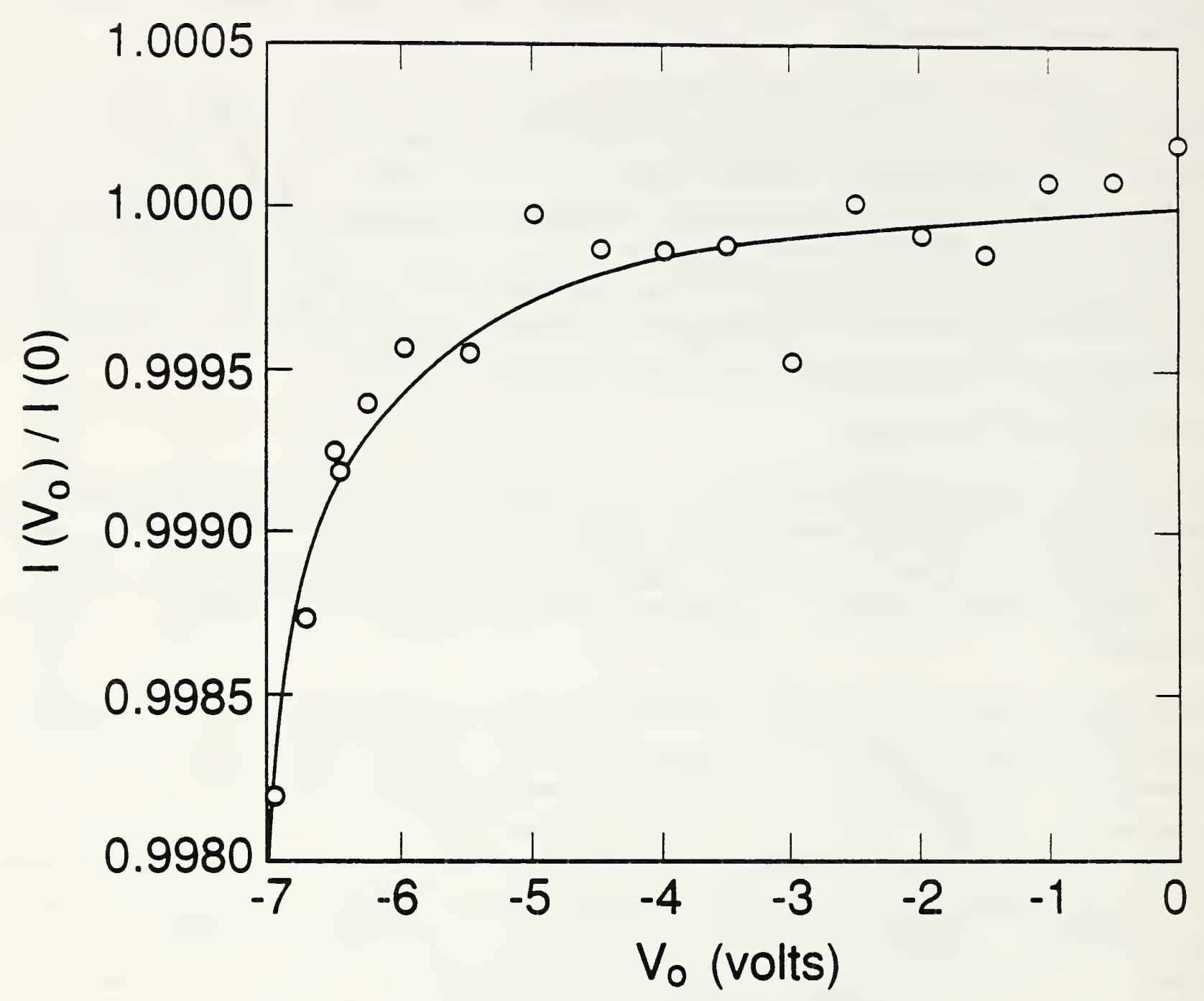

Figure 9. Ratio of the photocurrent $I\left(V_{0}\right)$ as a function of oxide-bias voltage $V_{0}$ to the photocurrent $\mathrm{I}(0)$ at zero-bias voltage as measured on a typical UDT UV100 silicon photodiode and as calculated with PC-1D for $\mathrm{S}=3.0 \times 10^{3} \mathrm{~cm} / \mathrm{s}$ and $\mathrm{N}_{s,}=1.38 \times 10^{12}$ $\mathrm{cm}^{-2}$. 
of $N_{s}$ and $S$, as can be seen in the figure.

These values of $N_{\Delta}$ and $S$ are used to simulate the internal quantum-deficiency spectra for a nominal UV100 photodiode in figure 10. The three different curves shown in figure 10 correspond to minority carrier lifetimes $\tau$ of $1 \mu \mathrm{s}, 10 \mu \mathrm{s}$, and $81 \mathrm{~ms}$, respectively, in the rear region of the photodiode. The lifetimes of $1 \mu \mathrm{s}$ and $10 \mu \mathrm{s}$ give internal quantum deficiencies at $700 \mathrm{~nm}$ that bracket the range measured for six typical UV100 type photodiodes [14], and the $81 \mathrm{~ms}$ lifetime is the longest lifetime that the default silicon material model of PC-1D will allow for $100 \Omega \mathrm{cm}$, p-type silicon with a recombination trap at mid-gap.

The region between the curves corresponding to $\tau=1$ and $\tau=10 \mu \mathrm{s}$ in figure 10 is a nominal internal quantum-deficiency spectrum that applies to UV100 type photodiodes and to QED 100 and QED 200 radiometers at zero reverse bias [3,12]. Figure 11 compares the spectrum for $\tau=1 \mu \mathrm{s}$ around its minimum in the 400 to $500 \mathrm{~nm}$ spectral region with spectra obtained for the cases where either the front-region surface recombination velocity is doubled, or the positive charge trapped in the oxide is halved. These are conservative limits to associate with the mass production of photodiodes in a controlled planar-silicon fabrication process. These should also be conservative limits for the effects of environmental stress, such as humidity, temperature, and irradiation with ultraviolet radiation, for UV100 photodiodes [15-16] made after 1986.

The curves in figure 11 can be used to estimate uncertainties to be associated with the nominal values of the internal quantum efficiency of the UV100 type photodiode. Summing in quadrature the differences between the nominal spectrum and the other two spectra in figure 11 produces a somewhat conservative estimate since increases in surface recombination velocity tend to be correlated with increases in trapped charge. In the region from 440 to $460 \mathrm{~nm}$, a nominal value of $0.0003 \pm 0.0003$ is a practical summary of the results of such an analysis. This result should be considered a one-standard-deviation equivalent, rather than a limit of error, since the whole analysis is based on the results obtained for a single device. Outside the 440 to $460 \mathrm{~nm}$ spectral region the uncertainties grow so rapidly that they are not really practical for high-accuracy applications.

There are a number of sources of error not yet considered, but since their effect scales with the magnitude of the internal quantum deficiency, they are all negligible with respect to \pm 0.0003 . This is illustrated in figure 12 for the uncertainty associated with the absorption coefficient of silicon in the 440-to $460-\mathrm{nm}$ spectral region, which is the largest uncertainty not considered so far.

Figure 12 compares the spectrum of figure 11 for $\tau=1 \mu \mathrm{s}$, which was based on the absorption-coefficient data in SIL_WEAK.ABS, with the same spectra calculated for identical parameters, but based on absorption-coefficient data (measured by Philipp [17], and described in Part I) that is stored in SIL_PHIL.ABS. When added in quadrature to the \pm 0.0003 assigned to the variations in $N_{\text {.. }}$ and $S$ from diode to diode, the effect of the uncertainty in the absorption coefficient is negligible. This might not remain true if a much larger wavelength interval were considered. 


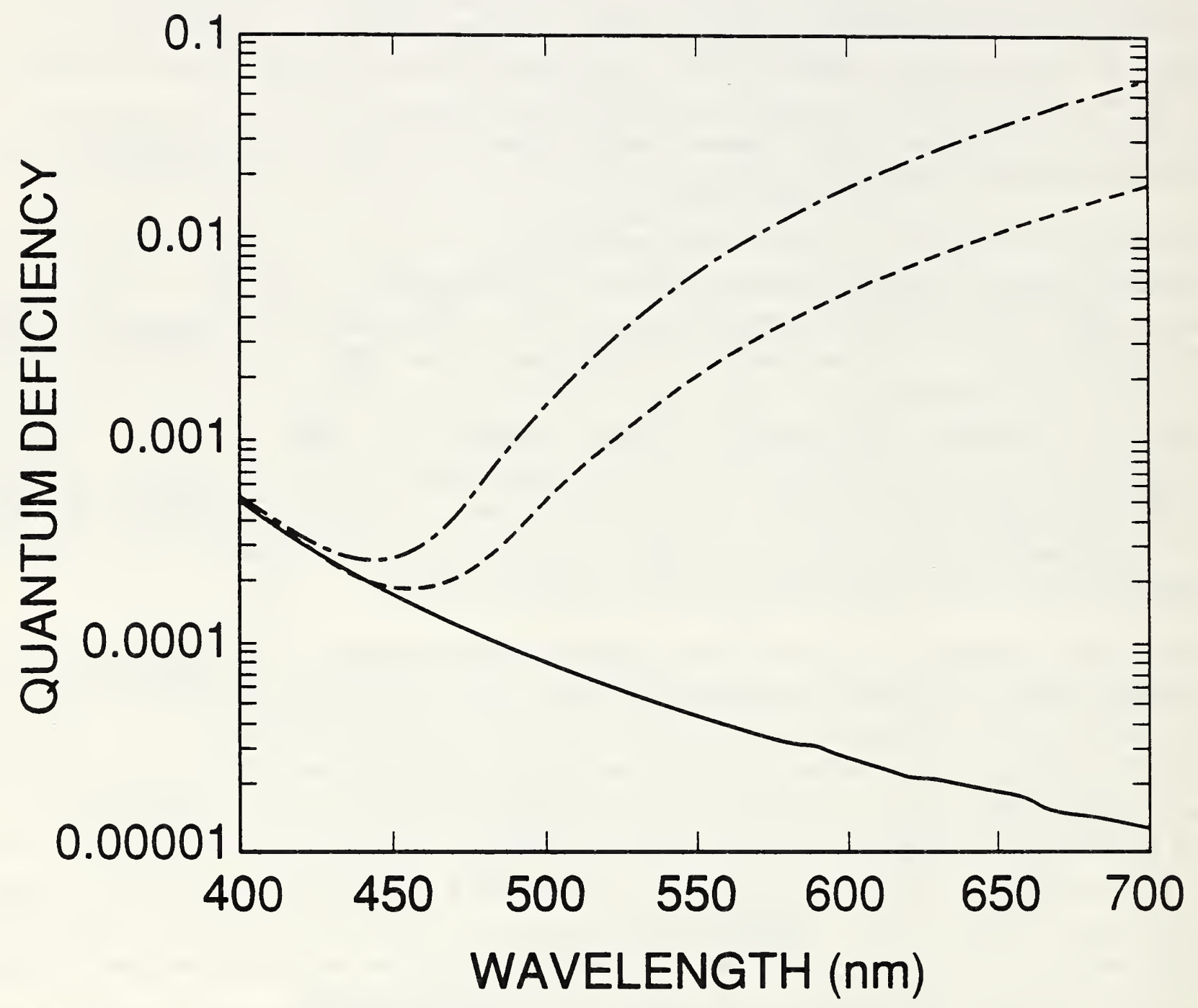

Figure 10. Internal quantum-deficiency spectra calculated by PC-1D for $S=3.0 \times 10^{5}$ $\mathrm{cm} / \mathrm{s}, \mathrm{N}_{\mathrm{ss}}=1.38 \times 10^{12} \mathrm{~cm}^{-2}$, and rear-region lifetimes $\tau$ of $81 \mathrm{~ms}$ (solid line), $10 \mu \mathrm{s}$ (dashed line), and $1 \mu \mathrm{s}$ (dot-dashed line). 


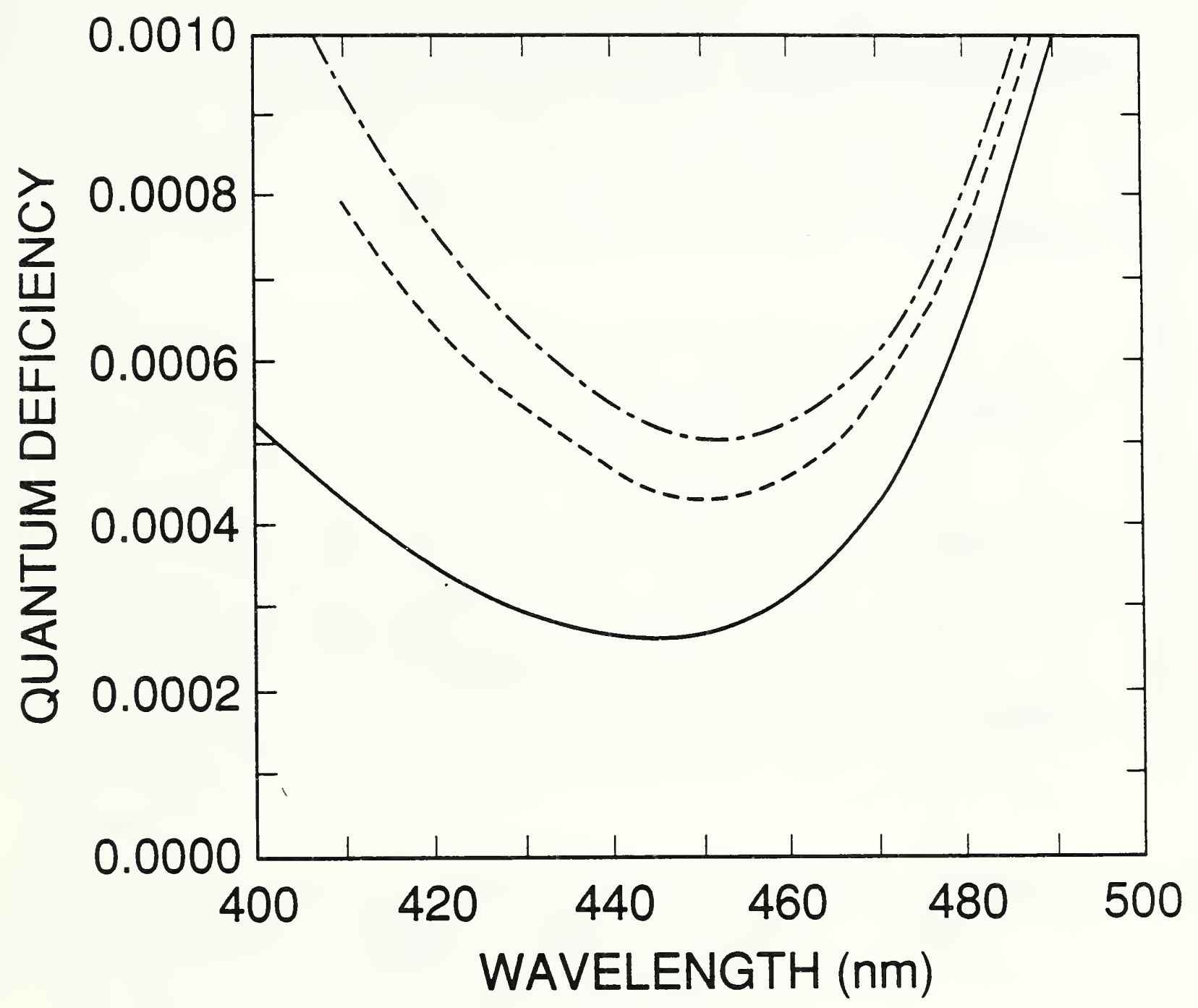

Figure 11. Internal quantum-deficiency spectra calculated by PC-1D for a rear-region lifetime $\tau=1 \mu \mathrm{s}$ with $\mathrm{S}=3.0 \times 10^{5} \mathrm{~cm} / \mathrm{s}$ and $\mathrm{N}_{\varrho},=1.38 \times 10^{12} \mathrm{~cm}^{-2}$ (solid line), with $\tau=1 \mu \mathrm{s}$ with $\mathrm{S}=6.0 \times 10^{5} \mathrm{~cm} / \mathrm{s}$ and $\mathrm{N}_{\varrho},=1.38 \times 10^{12} \mathrm{~cm}^{-2}$ (dashed line), and with $\tau$ $=1 \mu \mathrm{s}$ with $\mathrm{S}=3.0 \times 10^{5} \mathrm{~cm} / \mathrm{s}$ and $\mathrm{N}_{\jmath},=0.69 \times 10^{12} \mathrm{~cm}^{-2}$ (dot-dashed line). 


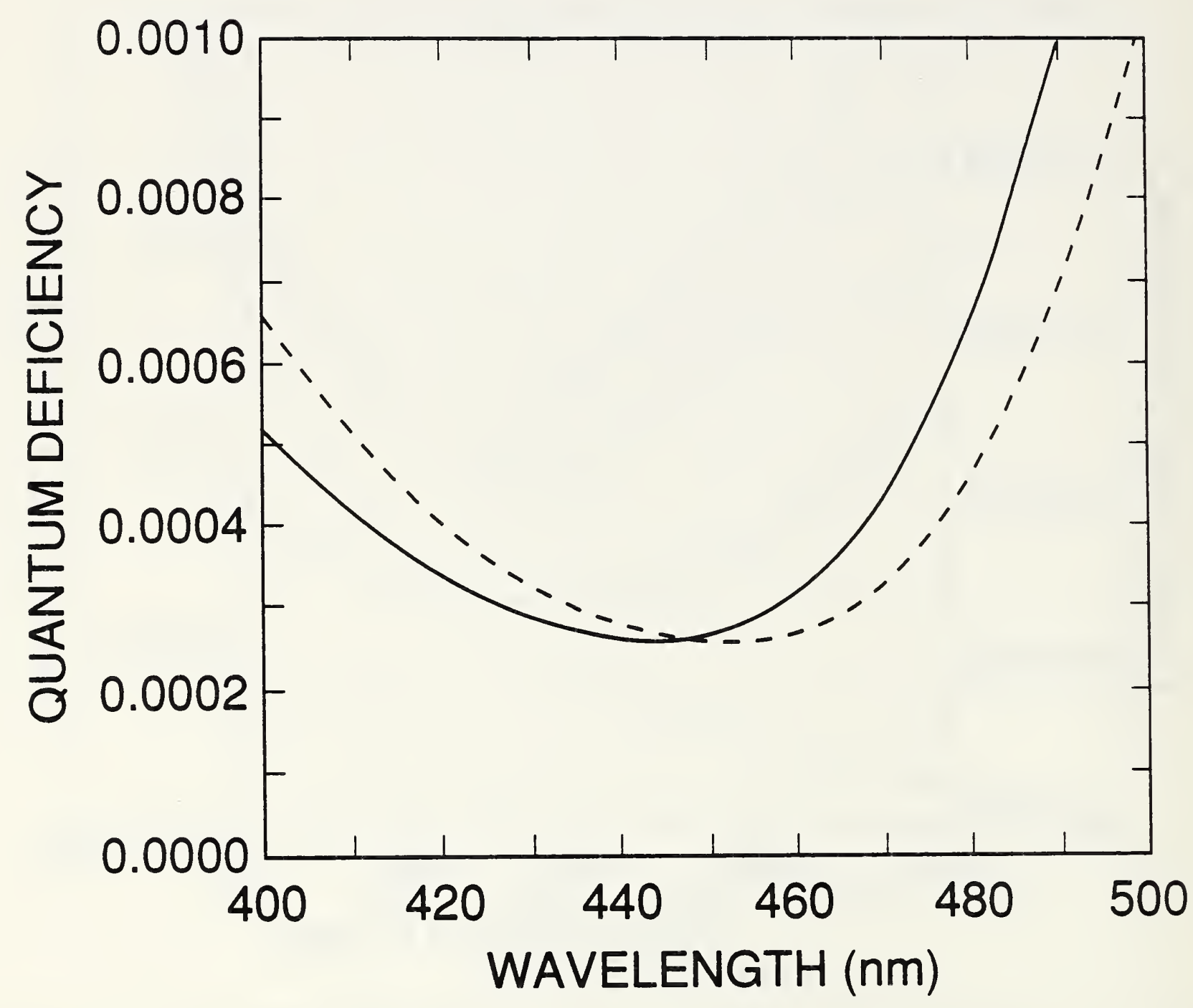

Figure 12. Comparison of the internal quantum-deficiency spectra calculated for a UV100 type photodiode for a rear-region lifetime $\tau=1 \mu \mathrm{s}$ with $\mathrm{S}=3.0 \times 10^{5} \mathrm{~cm} / \mathrm{s}$ and $\mathrm{N}_{s s}=$ $1.38 \times 10^{12} \mathrm{~cm}^{-2}$ with the absorption-coefficient data in the SIL.WEAK.ABS file (solid line), and with the absorption-coefficient data in the SIL_PHIL.ABS file (dashed line). The data in these files are described in more detail in Part I. 
Because of the large long-wavelength quantum deficiency shown in figure 10 , reverse bias is often applied to UV100 photodiodes and QED 100 and 200 radiometers when they are used at the longer wavelengths. By moving the depletion region toward the rear of the photodiode, the reverse bias forces the internal quantum deficiency to approach the lower quantum deficiency limit given by the $81 \mathrm{~ms}$ curve in figure 10 [12,18-19]. Reverse bias is also applied to eliminate the saturation type nonlinearity that is sometimes observed with photocurrents above a few microamperes. However, recent results [4] suggest that moderate bias levels can cause gain, resulting in an internal quantum efficiency in excess of unity. This effectively precludes the application of moderate reverse bias in high-accuracy applications.

Figure 13 plots a simulation of the decrease in the internal quantum deficiency as a function of reverse bias for the UV100 type photodiode at the 442, 476, and $514 \mathrm{~nm}$ laser lines. The feature in the curves for reverse-bias voltages less (more negative) than $-3.5 \mathrm{~V}$ is a symptom of a problem with PC-1D. It may mean that more than 150 finite elements are needed to maintain the level of accuracy achieved as the reverse bias is increased, but this is not certain. In any case, it not only invalidates the data for reverse-bias voltages more negative than $-3.5 \mathrm{~V}$, but it also casts some doubt on any conclusions drawn about the data at the less negative bias voltages as well. At best, figure 13 suggests that $480 \mathrm{~nm}$ is a practical upper limit to the wavelength at which reverse bias can be used to eliminate the effect of recombination in the rear region of the photodiode without risking gain. However, the best advice would be never to use a UV100 photodiode or QED radiometer with a bias voltage without first assuring experimentally that gain is not a problem.

\section{Conclusion}

Part II of this paper has demonstrated the use of Version 2 of PC-1D.EXE, RUN PC1D.BAT. MAKE_PRM.EXE, and READ_PDF.EXE to simulate various oxide-bias experiments of interest to high-accuracy applications of silicon photodiodes. In each case, the simulation was used to interpret an oxide-bias experiment, with different sorts of information being sought from the different experiments.

It was shown that the simulations can be used to derive a more accurate internal quantumdeficiency value from an oxide-bias experiment than that available from the conventional data reduction, and that an uncertainty can be associated with the value derived from the simulation based on how well the simulated oxide-bias data can be fitted to the experimental data. It was also shown that simulations can be used to determine nominal values for the front-surface recombination velocity and for the charge trapped in the front-surface oxide, and that values for these parameters can be used to determine nominal internal quantum-deficiency spectra for various types of silicon photodiodes.

As well as illustrating these general ideas, some more specific results were also obtained. Firstly, it was shown that a nominal internal quantum deficiency of $0.0003 \pm 0.0003$ is appropriate for the unbiased UDT UV100 type photodiode in the 440 to $460 \mathrm{~nm}$ spectral region. Secondly, it was shown that the shape of the internal quantum-deficiency spectrum of the 1337 type photodiode is quite independent of typical variations in doping 


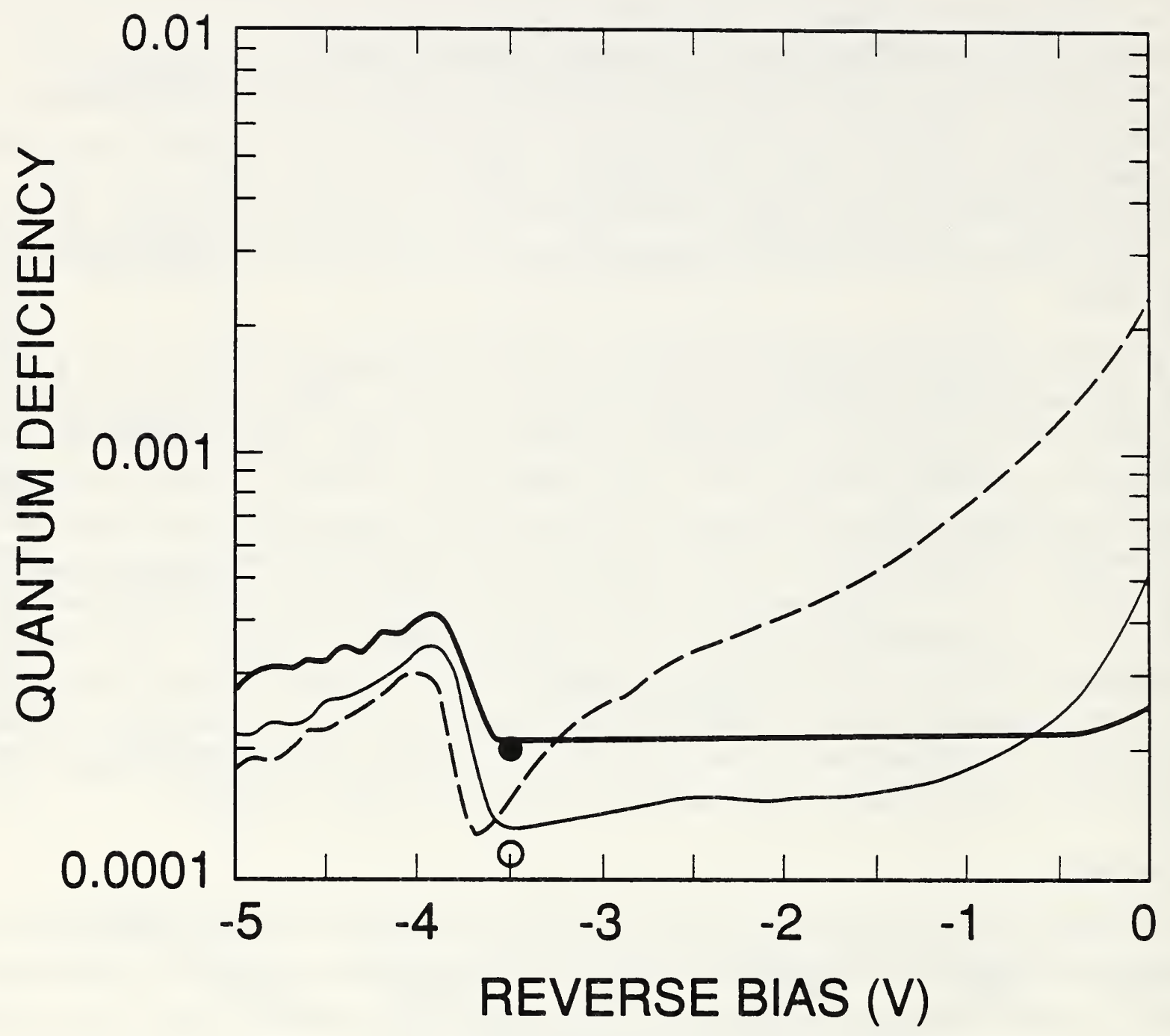

Figure 13. Simulation of the decrease in internal quantum-deficiency of a UV100 type photodiode with reverse bias at $442 \mathrm{~nm}$ (heavy solid line), $468 \mathrm{~nm}$ (light solid line), and $514 \mathrm{~nm}$ (dashed line). The values of $\mathrm{N}_{s,}, \mathrm{~S}$, and $\tau$ are as in figure 12. The feature near $-4 \mathrm{~V}$ is an artifact of the simulation. The open circle and closed circle are the internal quantum deficiencies at $442 \mathrm{~nm}$ and $468 \mathrm{~nm}$, respectively, for $\tau=81 \mathrm{~ms}$. The internal quantum deficiency at $514 \mathrm{~nm}$ for the same condition is too small to appear on the graph. 
profile. This result suggests that the 1337 type photodiode might serve to extrapolate high-accuracy calibrations obtained from cryogenic absolute radiometers or from QED type radiometers in the 440 to $460 \mathrm{~nm}$ spectral region to longer wavelengths with little or no loss of accuracy. Part III of this paper shows that this is the case. Finally, it was shown that reverse-bias simulations based on the programs described in Part I of this paper do not work well enough with inversion layer (induced junction) photodiodes to be useful for high-accuracy applications.

\section{References}

[1] Geist, J., J. Appl. Phys. 51, 1172 (1983).

[2] Zalewski, E. F., and Geist, J., Appl. Opt. 19, 1214 (1980).

[3] Geist, J., SPIE 1109, 246 (1989), provides a review of the oxide-bias, self-calibration technique.

[4] Köhler, R., Pello, R., and Bonhoure, J., Appl. Opt. 29, 4212 (1990).

[5] Geist, J., Migdall, A., and Baltes, H. P., Appl. Opt. 27, 3777 (1988).

[6] Weakliem, H. A., and Redfield, D., J. Appl. Phys. 50, 1491 (1979), and personal communication.

[7] Key, P. J., Fox, N. P., and Rastello, M. L., Metrolgia 21, 81 (1985).

[8] Geist, J., and Baltes, H. P., Appl. Opt. 28, 3929 (1989).

[9] Geist, J., Gardner, J. L., and Wilkinson, F. J., Phys. Rev. 42B, 1262 (1990).

[10] Geist, J., and Wang, C. S., Phys. Rev. 27B, 4841 (1983).

[11] Geist, J., Liang, E., and Schaefer, A. R., J. Appl. Phys. 52, 4879 (1981).

[12] Zalewski, E. F., and Duda, C. R., Appl. Opt. 22, 2867 (1983).

[13] Hansen, T., Phys. Scr. 18, 471 (1978).

[14] Houston, J. M., and Saunders, R. B., personal communication of data.

[15] Korde, R., and Geist, J., Solid-State Electronics 30, 89 (1987).

[16] Korde, R., and Geist, J., Appl. Opt. 26, 5284 (1987).

[17] Philipp, H. R., J. Appl. Phys. 43, 2835 (1972), and personal communication.

[18] Geist, J., Zalewski, E. F., and Schaefer, A. R., Appl. Opt. 19, 3795 (1980).

[19] Schaefer, A. R., Zalewski, E. F., and Geist, J., Appl. Opt. 22, 1232 (1983). 


\title{
Part III: INTERPOLATING AND EXTRAPOLATING INTERNAL QUANTUM-EFFICIENCY CALIBRATIONS
}

\author{
Jon Geist \\ NIST, Gaithersburg, MD 20899 \\ A. M. Robinson and C. R. James \\ University of Alberta, Alberta, Canada T6G 2G7 \\ Abstract
}

The semiconductor device modeling program PC-1D and the programs that support its use in high-accuracy modeling of photodiodes, all of which were described in Part I, are used to simulate the interpolation of high-accuracy internal quantum-efficiency calibrations in the spectral region between $450 \mathrm{~nm}$ and $850 \mathrm{~nm}$. Convenient interpolation formulae that depend only upon wavelength are derived. Uncertainty spectra for a number of sources of error are also derived. The formulae are normalized to experimental internalquantum efficiency calibrations in the 440 to $470 \mathrm{~nm}$ spectral region and at $860 \mathrm{~nm}$ and are used to interpolate the calibration values between these wavelengths. The results of the interpolations are compared with experimental calibration data that are available at a few wavelengths between 440 and $860 \mathrm{~nm}$. The disagreement between the interpolated and measured internal quantum-efficiency data is never worse than 0.0003 .

Key words: extrapolation; internal quantum-efficiency; interpolation; photodiode; silicon; visible

\section{Introduction}

Part II of this paper suggested that the Hamamatsu 1337 type photodiode might be conveniently used for interpolating or extrapolating high-accuracy quantum-efficiencies in the 400 to $900 \mathrm{~nm}$ spectral region. The idea of quantum-efficiency extrapolation was implicit in the first detailed description of how a silicon photodiode could be used (in this case in conjunction with a thermal detector having a spectrally flat responsivity) to realize a scale of absolute spectral responsivity [1], and interpolation was explicitly considered in Ref. [2]. Beyond these two publications, however, little progress has been reported in this area until recently.

Hoyt et al. [3] (using early results of the work described here) used three Hamamatsu 1337 type photodiodes to extrapolate a quantum-efficiency calibration obtained at 441.6 $\mathrm{nm}$ with a UDT QED 100 radiometer to $633.0 \mathrm{~nm}$, and reported an average difference of $0.04 \%$ compared to independent measurements based on their electrically calibrated cryogenic cavity radiometer. Even though $0.04 \%$ is quite good by conventional radiometric standards, it was a factor of two larger than the combined uncertainty estimate for the two measurement techniques being compared, and further study was proposed by Hoyt et al. More recently, Zalewski and Hoyt [4] have reported a more direct test of the accuracy of the use of the Hamamatsu 1337 type photodiode to extrapolate quantum efficiency values. The results of this test agreed to within the combined estimated uncertainty, and two errors were discovered in the earlier work. When these errors were corrected, agreement 
within the estimated uncertainty of the earlier intercomparison was obtained.

The purpose of Part III is to develop formulae and uncertainty estimates for interpolating and extrapolating internal quantum-efficiency calibrations with Hamamatsu 1337 photodiodes. It will be shown that this type of photodiode has characteristics that suit it particularly well for this task over the 440 to $860 \mathrm{~nm}$ spectral region. The remainder of Part III is organized as follows: Section 2 investigates the errors associated with the use of relations that are only approximate for the 1337 type photodiode, but which simplify interpolation and extrapolation of the internal quantum deficiency (one minus the internal quantum efficiency) for this type of photodiode. Section 3 investigates the various sources of error associated with the actual calculation of internal quantum-deficiency spectra using the semiconductor-device modeling program PC-1D and the support programs described in Part I. Finally, section 4 compares the results obtained here with experimental measurements and demonstrates agreement within 0.0003 .

\section{Errors Associated with the Quantum-Deficiency Approximations}

For the purposes of extrapolating and interpolating the internal quantum efficiency of Hamamatsu 1337 type photodiodes, the internal quantum deficiency can be approximated by a function $\delta\left(\lambda, N_{s s}, S, \tau_{r}\right)$ of wavelength $\lambda$ and of three recombination-related variables:

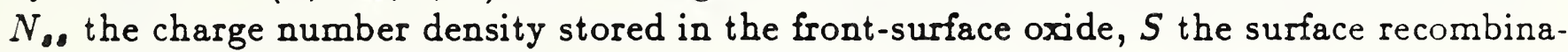
tion velocity at the oxide-silicon interface, and $\tau_{r}$ the minority-carrier lifetime in the rear region.

For silicon photodiodes, $\delta\left(\lambda, N_{\lrcorner}, S, \tau_{r}\right)$ can be approximated by a function that has properties that make it particularly useful for extrapolating and interpolating quantum deficiency measurements. The approximate equation for $\delta\left(\lambda, N_{s s}, S, \tau_{r}\right)$ is

$$
\begin{aligned}
\delta\left(\lambda, N_{. \ell}, S, \tau_{r}\right) & =K_{f}\left(N_{, \varrho}, S\right) \delta_{f}(\lambda) \\
& +K_{r}\left(\tau_{r}\right) \delta_{r}(\lambda)
\end{aligned}
$$

where

$$
\delta_{r}(\lambda)=0
$$

for $\lambda$ less than some wavelength $\lambda_{0}$.

To see how this is useful for interpolating quantum deficiency values, suppose that $\delta\left(\lambda_{f}, N_{s s}\right.$, $\left.S, \tau_{r}\right)$ has been measured at wavelength $\lambda_{f}<\lambda_{0}$, and $\delta\left(\lambda_{r}, N_{o,}, S, \tau_{r}\right)$ has been measured at wavelength $\lambda_{r}>\lambda_{0}$. The values for $K_{f}\left(N_{, g}, S\right)$ and $K_{r}\left(\tau_{r}\right)$ can then be determined from 


$$
K_{f}\left(N_{s s}, S\right)=\frac{\delta\left(\lambda_{f}, N_{s s}, S, \tau_{r}\right)}{\delta_{f}\left(\lambda_{f}\right)}
$$

and

$$
K_{r}\left(\tau_{r}\right)=\frac{\delta\left(\lambda_{r}, N_{s s}, S, \tau_{r}\right)-K_{f}\left(N_{s s}, S\right) \delta_{f}\left(\lambda_{r}\right)}{\delta_{r}\left(\lambda_{r}\right)}
$$

and these values can be used in eq (1) to interpolate the quantum deficiency between $\lambda_{f}$ and $\lambda_{r}$. In the above equations, $\delta_{f}(\lambda)$ and $\delta_{r}(\lambda)$ are functions only of wavelength, $\lambda_{f}$ and $\lambda_{r}$ are the wavelengths at which the internal quantum deficiency is to be normalized, and $\lambda_{0}$ will generally fall between $600 \mathrm{~nm}$ and $650 \mathrm{~nm}$. The subscript $f$ stands for the front of the photodiode since this is the location of the recombination mechanisms that cause $\delta_{f}(\lambda)$ to exceed zero. The subscript $r$ stands for the rear of the photodiode since this is the location of the recombination mechanism that cause $\delta_{r}(\lambda)$ to exceed zero. Therefore, $\delta_{f}(\lambda)$ and $\delta_{r}(\lambda)$ will be called the front-region and rear-region internal quantum-deficiency spectra, respectively. The functional dependence of $K_{f}$ and $K_{r}$ will be dropped from the notation for the remainder of this paper.

Exactly how accurate eqs (1-3) are depends upon the particular type of photodiode and the range covered by the parameters. Figure 1 illustrates the accuracy of eq (1) for the 1337 type photodiode (modeled as described in Part II of this paper) over the 400 to $900 \mathrm{~nm}$ spectral region for an internal quantum-deficiency spectrum that is a practical upper limit for this type of photodiode. Figure 1 compares the internal quantum-deficiency spectra $\delta(\lambda, 0,71264 \mathrm{~cm} / \mathrm{s}, \infty)$ and $\delta(\lambda, 0,0,1 \mathrm{~ms})$ with their sum, and with the internal quantumdeficiency spectrum $\delta(\lambda, 0,71264 \mathrm{~cm} / \mathrm{s}, 1 \mathrm{~ms})$. The latter spectrum and the sum of the two first spectra differ by less than $2 \times 10^{-5}$ over the entire $400 \mathrm{~nm}$ spectral region, and cannot be distinguished at the scale of the figure. (Any errors with absolute values less than $2 \times 10^{-5}$ are negligible for the purposes of this paper.)

Figure 2 illustrates the accuracy of eqs (1-3) over the 400 to $900 \mathrm{~nm}$ spectral region for the case where $\tau_{r}=\infty$. The spectra in figure 2 were calculated for the range of parameters listed in table 1. Cases 1-3 in that table are based on the front-region doping distribution $N_{A}(x) / N_{A}(0)$ shown as the dashed curve in Figure 3, and Case 4 is based on that shown as the solid curve in the same figure. Of six doping distributions measured for different 1337 type photodiodes, the two shown in Figure 3 produce the largest change in shape of the internal quantum-deficiency spectrum.

The shapes of the front-region internal quantum-deficiency spectra in figure 2 are compared in figure 4 by normalizing all of the spectra to 0.01 at $440 \mathrm{~nm}$ and by plotting their differences relative to the normalized spectrum for Case 2. Cases 1 and 3 compared with Case 2 shows how the shape of $\delta_{f}(\lambda)$ changes when its magnitude is increased by over a factor of three in association with either a substantial decrease or a substantial increase in $N_{6,}$. (Cases 1 and 3 bracket a number of cases that were examined in which $N_{\text {,. }}$ and $S$ 


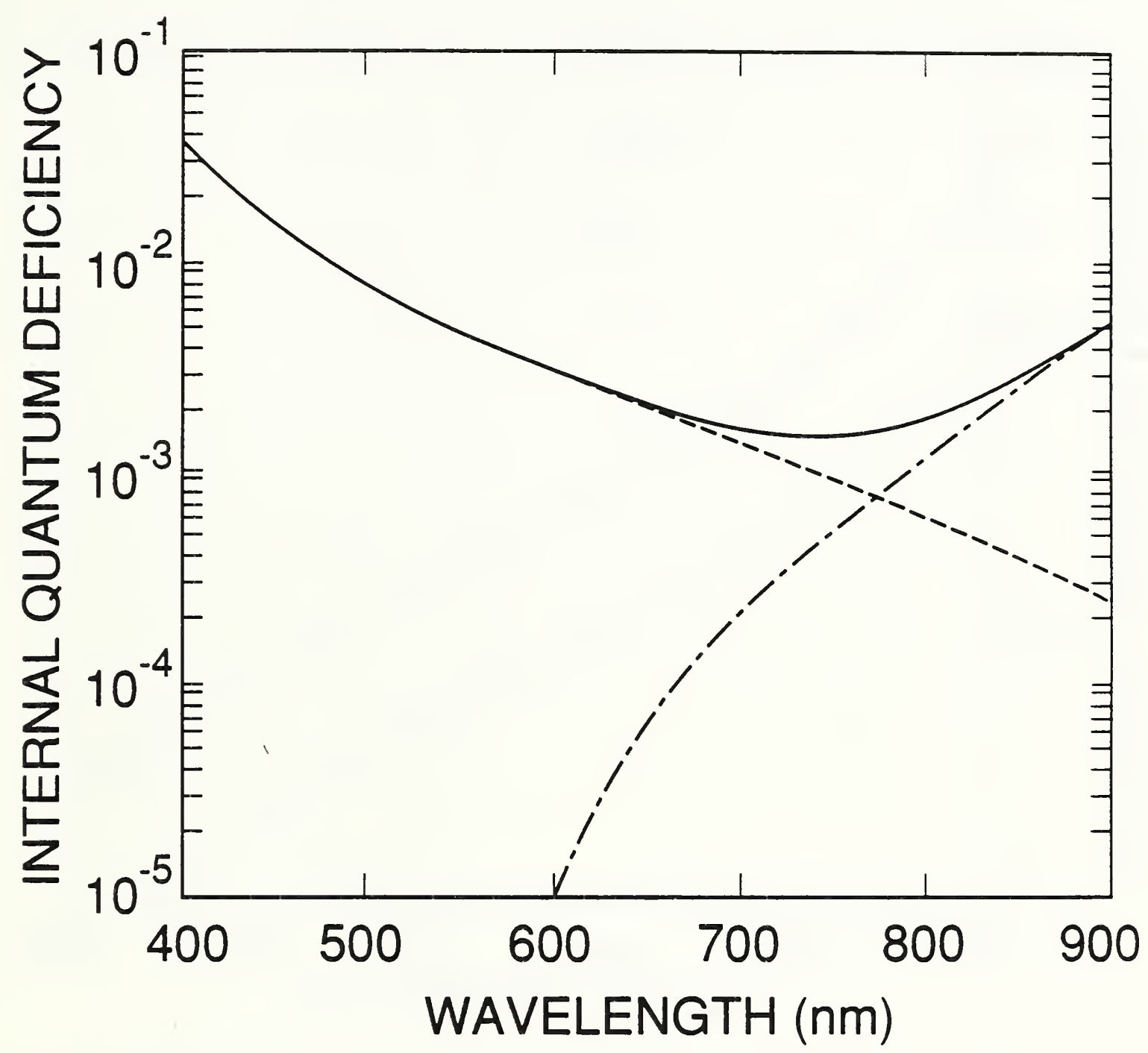

Figure 1. The quantum-deficiency spectra $\delta(\lambda, 0,71264 \mathrm{~cm} / \mathrm{s}, \infty)$ (dashed line) and $\delta(\lambda, 0.0 .1$ $\mathrm{ms})$ (dot-dashed line) and their sum (solid line). 


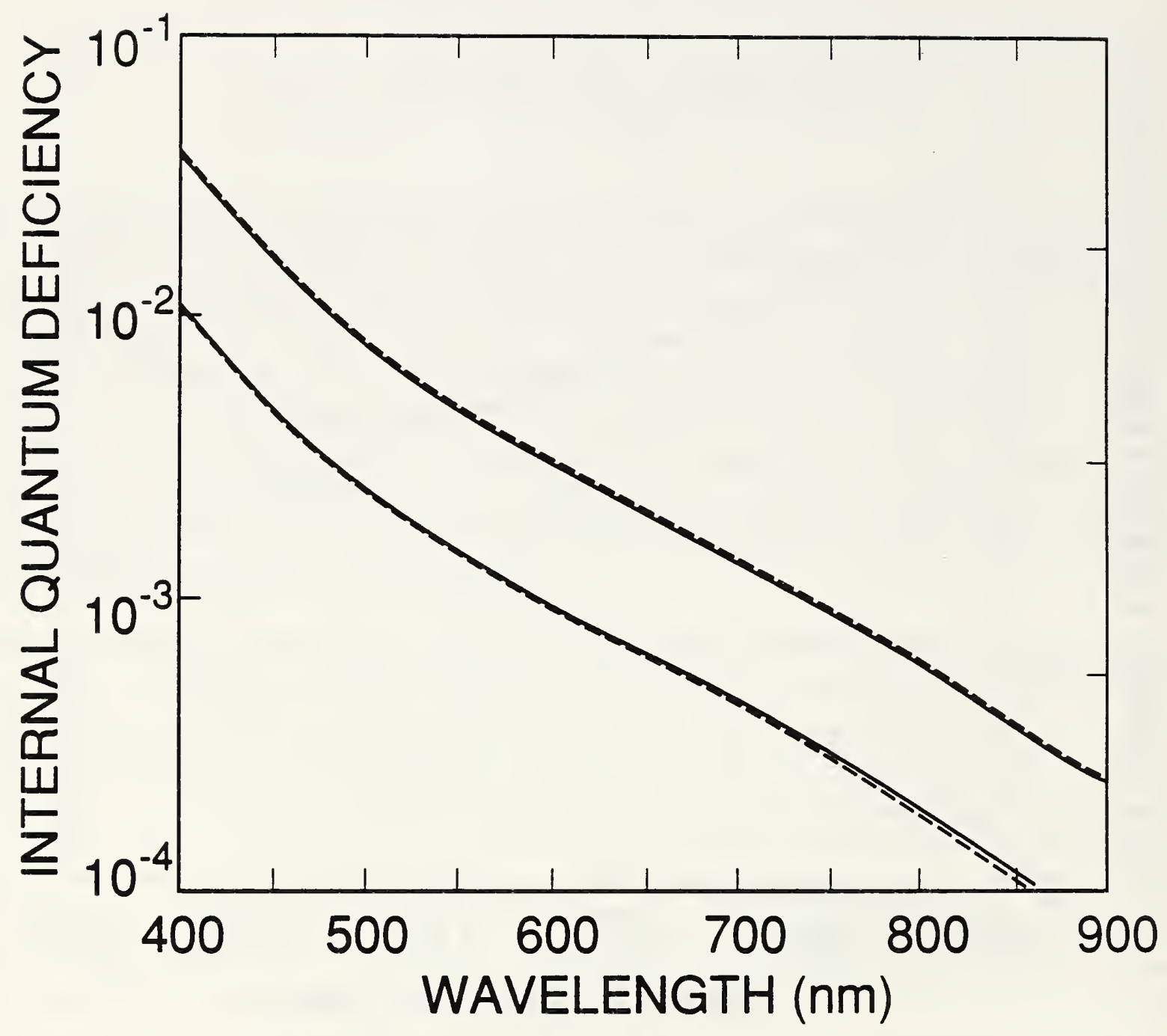

Figure 2. The quantum-deficiency spectra $\delta\left(\lambda, N_{\iota .}, S, \tau_{r}\right)$ for the conditions (Case 1 upper dashed line; Case 2 - lower dashed line; Case 3 - upper solid line; Case 4 - lower solid line) listed in table 1. 
Table 1. Recombination-related parameters used with the doping distributions shown in figure 3 to model the quantum-deficiency spectra of Hamamatsu 1337 type photodiodes.

$\begin{array}{ccccc}\text { CASE } & \begin{array}{c}N_{s s} \\ \left(\mathrm{~cm}^{-3}\right)\end{array} & \begin{array}{c}\mathrm{S} \\ \left(10^{5} \mathrm{~cm} / \mathrm{s}\right)\end{array} & \begin{array}{c}\tau_{r} \\ (\mathrm{~s})\end{array} & N_{A}(x) \\ 1 & 0 & 0.71264 & \infty & \text { dashed curve } \\ 2 & -3 \times 10^{12} & 1.83500 & \infty & \text { dashed curve } \\ 3 & -1 \times 10^{13} & 100.000 & \infty & \text { dashed curve } \\ 4 & -4 \times 10^{12} & 1.56000 & \infty & \text { solid curve }\end{array}$




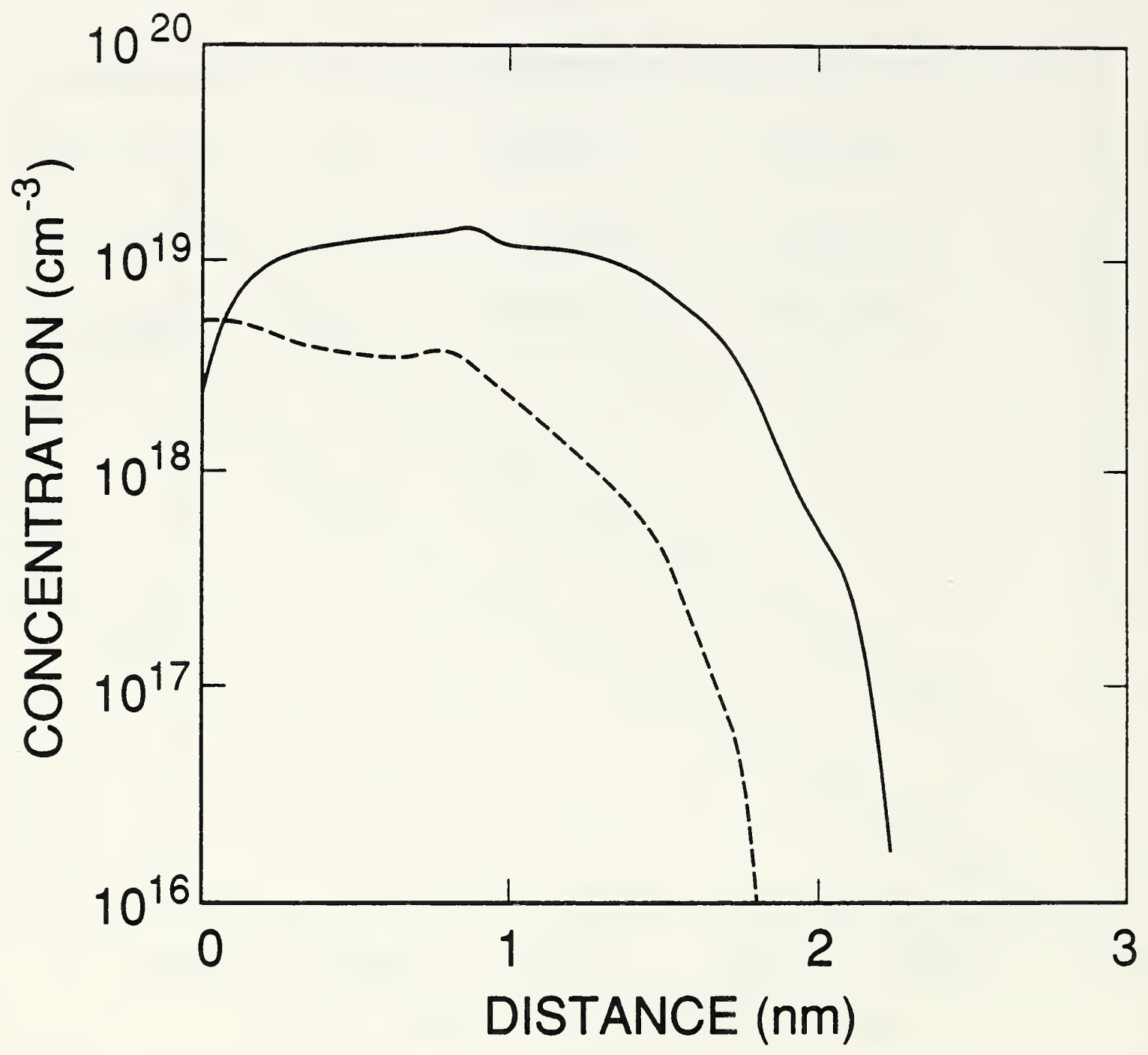

Figure 3. The front-region doping distributions $N_{A}(x)$ used to calculate the front-region internal quantum-deficiency curves in figure 2. 


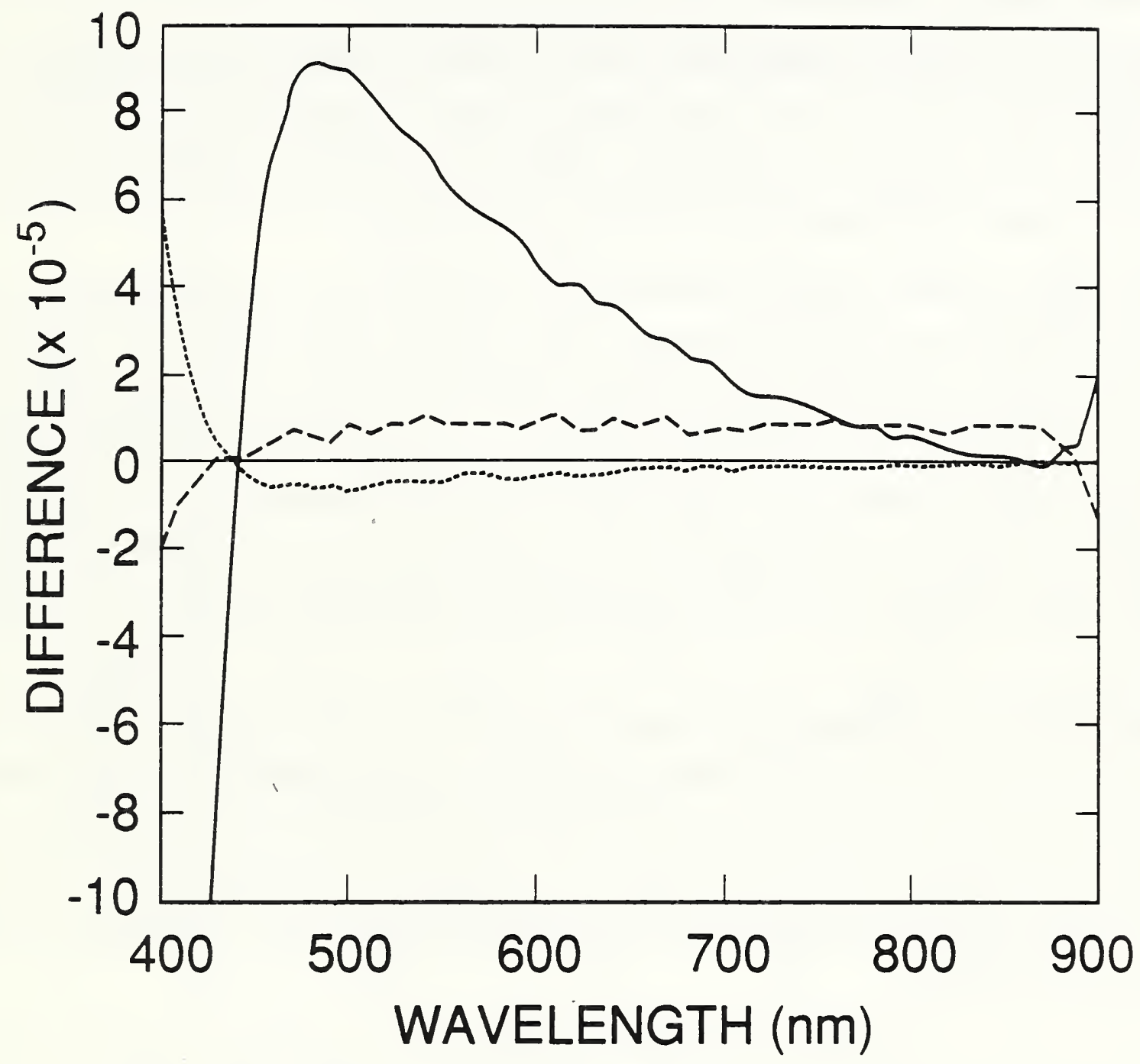

Figure 4. Differences between the internal quantum-deficiency spectra of figure 2 for Cases 1 (dashed line), 3 (dotted line), and 4 (solid line) when normalized to 0.01 at $440 \mathrm{~nm}$, and that for Case 2 when normalized to 0.01 at $440 \mathrm{~nm}$. 
were independently varied within the range of values shown in table 1.)

Case 4 compared with Case 2 shows how the shape of $\delta_{f}(\lambda)$ changes when the dopant distribution is changed from the dashed curve to the solid curve in figure 4 . This effect is much larger than that associated with varying $N_{\text {s. }}$ and $S$.

The three spectra in figure 4 characterize the uncertainties to be associated with the diodeto-diode variations in $N_{s s}, S$, and $N_{A}(x)$ when any one of the spectra in that figure is used as $\delta_{f}(\lambda)$ with $K_{f}=0.01$ in eqs (1) and (3). These spectra should be multiplied by $K_{f} / 0.01$ if $K_{f} \neq 0.01$. For the purposes of this paper, the $\delta\left(\lambda, N_{s}, S, \infty\right)$ spectrum corresponding to Case 4 in table 1 will be used for $\delta_{f}(\lambda)$ in eqs (1) and (3).

The front-region internal quantum deficiency also depends upon two front-region recombination mechanisms not considered above. These are the Auger and band-to-band mechanisms that become important in the heavily doped front region of the photodiode. Both effects were included in all of the spectra described so far by using the default cross sections that are built into $\mathrm{PC}-1 \mathrm{D}$ for these recombination mechanisms. The $\mathrm{PC}-1 \mathrm{D}$ default values for the Auger recombination cross sections give an internal quantum-deficiency spectrum with a value of $9 \times 10^{-6}$ at $400 \mathrm{~nm}$. The shape of this spectrum is well modeled by $\delta_{f}(\lambda)$ [5]. In fact, an uncertainty of $\pm 100 \%$ can be tolerated in the Auger cross sections without causing an error as large as $2 \times 10^{-5}$ anywhere within the 400 to $900 \mathrm{~nm}$ spectral region.

For the doping profile shown as the solid curve in figure 3, the PC-1D default values for the band-to-band recombination cross sections give the lifetimes shown in figure 5 as a function of distance from the oxide-silicon interface in the photodiode. These lifetimes give an internal quantum-deficiency spectrum with a value of $6 \times 10^{-5}$ at $400 \mathrm{~nm}$. This spectrum is not as well modeled by $\delta_{f}(\lambda)$ as is the spectrum for Auger recombination, but an uncertainty of $\pm 50 \%$ in the band-to-band recombination cross sections can be tolerated without raising the error associated with this uncertainty to $2 \times 10^{-5}$ anywhere in the 400 to $900 \mathrm{~nm}$ spectral region.

Now consider $\delta_{r}(\lambda)$. Figures 6 and 7 illustrate the accuracy of eqs $(1)$ and $(3)$ over the 400 to $900 \mathrm{~nm}$ spectral region for the case where $S=0$. (Note that when $S=0$, the internal quantum-deficiency spectrum is independent of $N_{s .}$.) The spectra in figure 6 were calculated for $\tau_{r}=1 \mathrm{~ms}$ and $10 \mathrm{~ms}$, with $N_{\mu}=S=0$. For the purposes of this paper, $\delta_{r}(\lambda)$ will be set to zero in eqs (2) and (3) for $\lambda \leq 610 \mathrm{~nm}$, and the spectrum for $\tau_{r}=1$ ms will be used for $\delta_{r}(\lambda)$ for $\lambda>610 \mathrm{~nm}$.

The shapes of the spectra in figure 6 are compared in figure 7 by normalizing them to 0.002 at $860 \mathrm{~nm}$ and plotting their differences relative to the spectrum for $\tau_{r}=1 \mathrm{~ms}$. The normalization value of 0.002 was chosen as a practical upper limit. The difference spectrum in figure 7 characterizes the uncertainties to be associated with the variations in rear-region lifetime when the spectrum for $\tau_{r}=1 \mathrm{~ms}$ is used as $\delta_{r}(\lambda)$ with $K_{r}=0.002$ in eqs (2) and (3). This spectrum should be multiplied by $K_{r} / 0.002$ if $K_{r} \neq 0.002$.

A convenient function of wavelength has been fitted to the spectrum corresponding to Case 4 in table 1 , and another to the spectrum for $\tau_{r}=1 \mathrm{~ms}$ in figure 6 . These functions are 


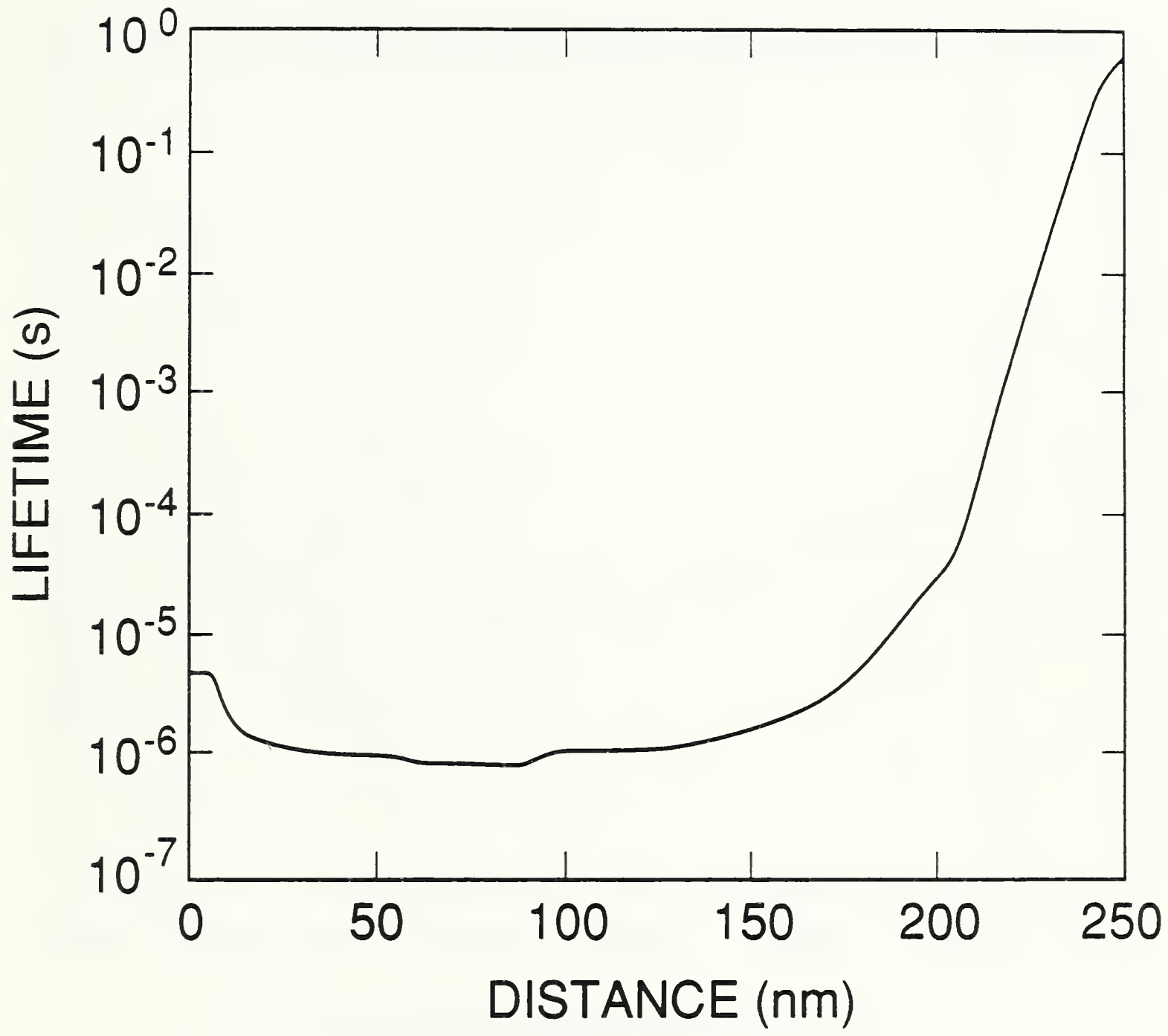

Figure 5. The default, band-to-band recombination lifetime profile calculated by PC-1D for the doping distribution shown as the solid curve in figure 3 . 


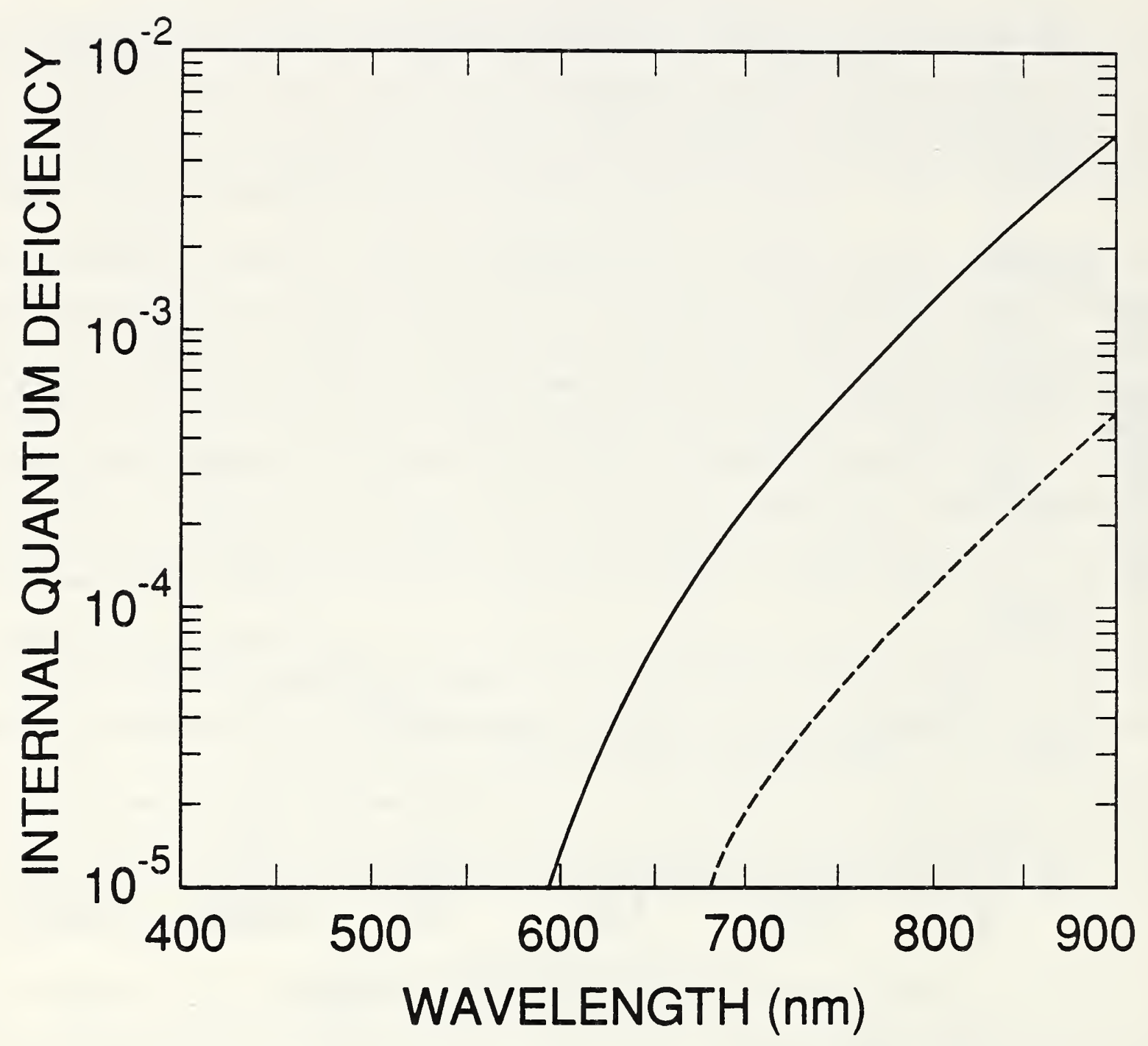

Figure 6. The internal quantum-deficiency spectra $\delta\left(\lambda, N_{\Perp s}, S, \tau_{r}\right)$ for $\tau_{r}=1 \mathrm{~ms}$ (solid line), and $\tau_{r}=10 \mathrm{~ms}$ (dashed line) with $N_{\text {.. }}=S=0$. 


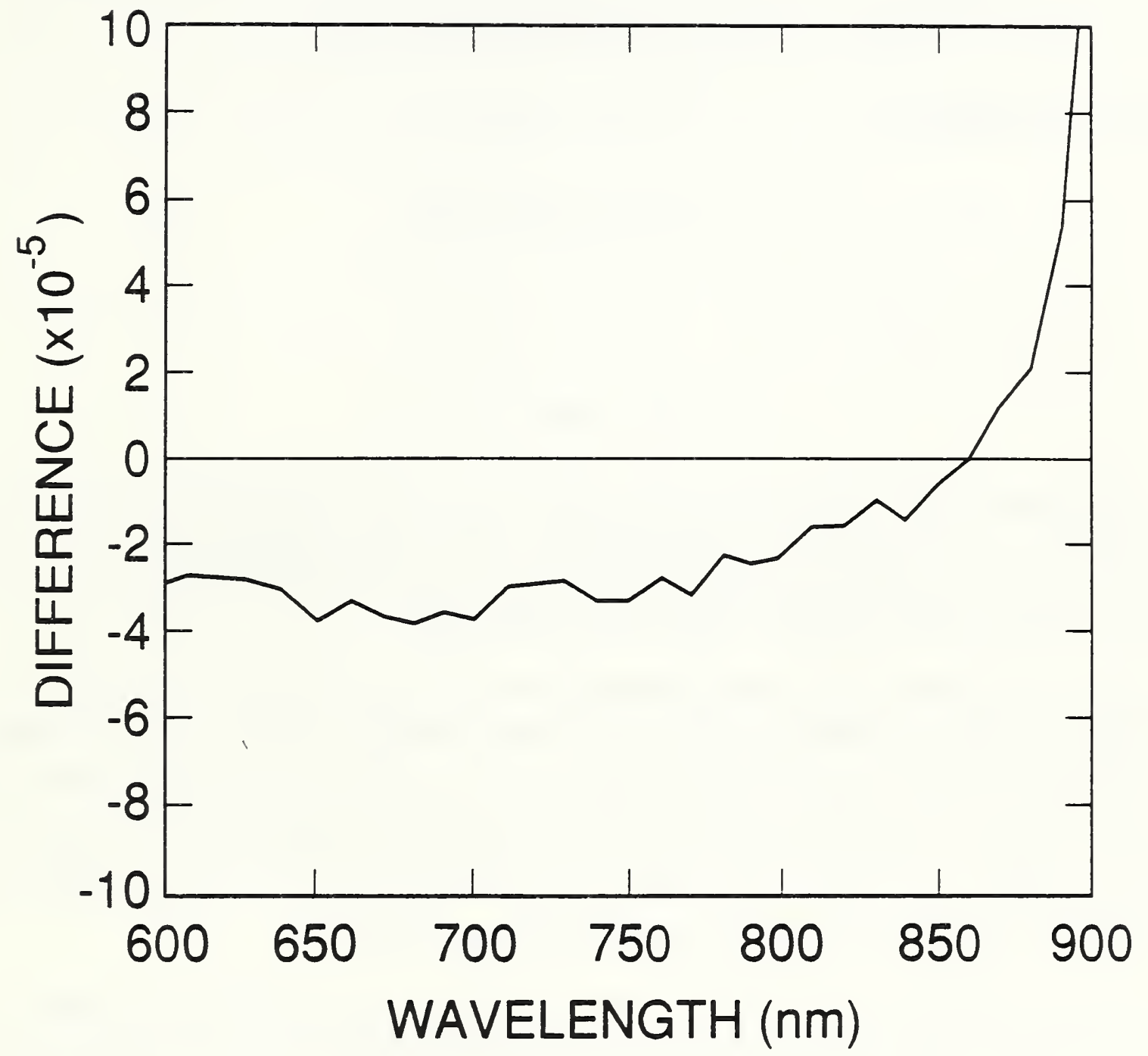

Figure 7. Differences between the internal quantum-deficiency spectra of figure 6 when normalized to 0.002 at $860 \mathrm{~nm}$. 


$$
\delta_{f}(\lambda)=A_{0}\left[X(\lambda)+A_{1} X^{2}(\lambda)+A_{2} Y(\lambda)\right]
$$

and

$$
\delta_{r}(\lambda)=A_{0}\left[1 / X(\lambda)-A_{2} / Y(\lambda)\right]
$$

respectively, where

$$
X(\lambda)=\exp \left(-\lambda / \lambda_{0}\right)
$$

and

$$
Y(\lambda)=\exp \left(-\left(\lambda / \lambda_{2}\right)^{2}\right)
$$

The appropriate values for $A_{0}, A_{1}, A_{2}, \lambda_{0}$, and $\lambda_{2}$ for eqs (4) and (5) are listed in table 2. The function in eq (5) becomes negative for $\lambda<620 \mathrm{~nm}$, in which case eq (5) is replaced with $\delta_{r}(\lambda)=0$ as mentioned earlier. The values of $A_{0}$ in table 2 normalize $\delta_{f}(\lambda)$ to 0.01 at $440 \mathrm{~nm}$, and $\delta_{r}(\lambda)$ to 0.002 at $860 \mathrm{~nm}$, respectively.

With $K_{f}=0.5516405$, eq (4) fits the spectrum for $N_{s o}=-3 \times 10^{12} \mathrm{~cm} / \mathrm{s}$ in figure 4 with a residual standard deviation of $2.9 \times 10^{-6}$ over the 420 to $900 \mathrm{~nm}$ spectral region and never differs from that spectrum by more than $7 \times 10^{-6}$ over the same spectral region. This translates to less than $10 \mathrm{ppm}$ when the internal quantum deficiency is normalized to 0.01 at $440 \mathrm{~nm}$; thus the error associated with the use of eq (4) is negligible for the purposes of this paper. The larger variations below $420 \mathrm{~nm}$ are negligible with respect to uncertainties already identified and others discussed below.

With $K_{r}=1.685081$, eq (5) fits the spectrum shown as a solid line in figure 6 with a residual standard deviation of $1.4 \times 10^{-5}$ over the 610 to $900 \mathrm{~nm}$ spectral region, and never differs from that spectrum by more than $2.5 \times 10^{-5}$ over that same spectral range. This translates to less than $1.7 \times 10^{-5}$ when the quantum deficiency is normalized to 0.002 at $440 \mathrm{~nm}$; thus the error associated with the use of eq (5) is also negligible for the purposes of this paper.

3. Errors Associated with Quantum-Deficiency Calculations

Two types of error associated with the quantum-deficiency values calculated by PC-1D can be distinguished: 1) numerical errors associated with the algorithms used by PC-1D and the limited number of finite elements available to PC-1D, and 2) physical errors associated with approximations and simplifications in the physical models used with, or built into PC-1D. Both types are considered in this section.

It was shown in Ref. [5] that $\delta_{f}(\lambda)$ is well approximated by 
Table 2. Values of the parameters to be used in eq (4) for extrapolating a short wavelength internal quantum-deficiency value to longer wavelengths and in eq (5) for extrapolating a long wavelength internal quantum-deficiency value to shorter wavelengths.

$\begin{array}{ccc}\text { Parameter } & \text { eq }(4) & \text { eq }(5) \\ A_{0} & 1.38002 \times 10^{2} & 2.02157 \times 10^{-8} \\ A_{1} & 1.47530 \times 10^{2} & 3.51764 \times 10^{3 ?} \\ A_{2} & 8.07476 \times 10^{-5} & 0.0 \\ \lambda_{0} & 4.39312 \times 10^{1} \mathrm{~nm} & 7.53991 \times 10^{1} \mathrm{~nm} \\ \lambda_{2} & 4.27998 \times 10^{2} \mathrm{~nm} & 1.66889 \times 10^{8} \mathrm{~nm}\end{array}$




$$
\begin{gathered}
\delta_{f}(\lambda)=\frac{\Gamma[I(0)-I(\alpha(\lambda))]}{1+\Gamma I(\alpha(\lambda))}, \\
I\left(\alpha(\lambda)=\int_{0}^{x_{1}} \hat{k}(x) \exp (-\alpha(\lambda) x) d x\right. \\
\hat{k}(x)=k(x) / k(0), \\
k(x)=1 /\left(m_{0}(x) D_{m}(x)\right), \\
\Gamma=S / D_{m}(0),
\end{gathered}
$$

where $\alpha(\lambda)$ is the absorption-coefficient spectrum of silicon, $x$ is the distance from the oxide-silicon interface toward the rear of the photodiode, $m_{0}(x)$ is the equilibrium minoritycarrier concentration at the point $x$ in the front region of the photodiode, and $D_{m}(x)$ is the equilibrium diffusion constant for the minority carriers at the point $x$. Note that the sign in the numerator in eq $(8)$ is correct and that the sign is incorrect in Ref. [5]. Also, note that $f(\lambda)$ becomes very insensitive to $x_{1}$ as $x_{1}$ increases beyond some critical value. The point where the electron and hole concentrations cross is a convenient choice for $x_{1}$, but smaller values would work as well.

From the point of view of PC-1D, the kernel $k(x)$ in eq (11) is given by

$$
k(\boldsymbol{x})=q M_{0}(\boldsymbol{x}) /\left(n_{\boldsymbol{i e}}^{2}(\boldsymbol{x}) k T \mu_{m}(\boldsymbol{x})\right),
$$

where $M_{0}(x)$ is the equilibrium majority carrier concentration in the front region, $n_{\text {ie }}(x)$ is the effective intrinsic carrier concentration [6], which in PC-1D includes the effect of Fermi-Dirac statistics, $\mu_{m}(x)$ is the minority-carrier mobility as a function of position in the front region, $k$ is the Boltzmann constant, and $T$ is the temperature of the photodiode.

Figure 8 compares $\exp (-\alpha(400 \mathrm{~nm}) x)$ with the equilibrium functions $M_{0}(x), n_{\text {ie }}(x)$, and $\mu_{m}(x)$ as calculated by PC-1D over the range $0<x<200 \mathrm{~nm}$ for the same photodiode model used to calculate the quantum-deficiency spectra in figure 3 for Case 2 of table 1 . Equations (8-12), figure 8, and figure 3 show why the shape of $\delta_{f}(\lambda)$ is very insensitive to $N_{\text {s. }}$ while being quite sensitive to the shape of $N_{A}(x) / N_{A}(0)$. Since the width of the accumulation layer created by $N_{\text {o, }}$ is small compared to the distance over which $\exp (-\alpha(\lambda) x)$ changes significantly for $\lambda \geq 400 \mathrm{~nm}$, changes in $N_{\text {o. }}$ have the effect of multiplying the integral in eq (9) by a constant factor independent of $\lambda \geq 400 \mathrm{~nm}$. On the other hand, the changes in $N_{\boldsymbol{A}}(x) / N_{\boldsymbol{A}}(0)$ shown in figure 3 extend well into the region where $\exp (-\alpha(\lambda) x)$ varies significantly from unity for $\lambda=400 \mathrm{~nm}$. As $\lambda$ is increased above $400 \mathrm{~nm}$, the shape 


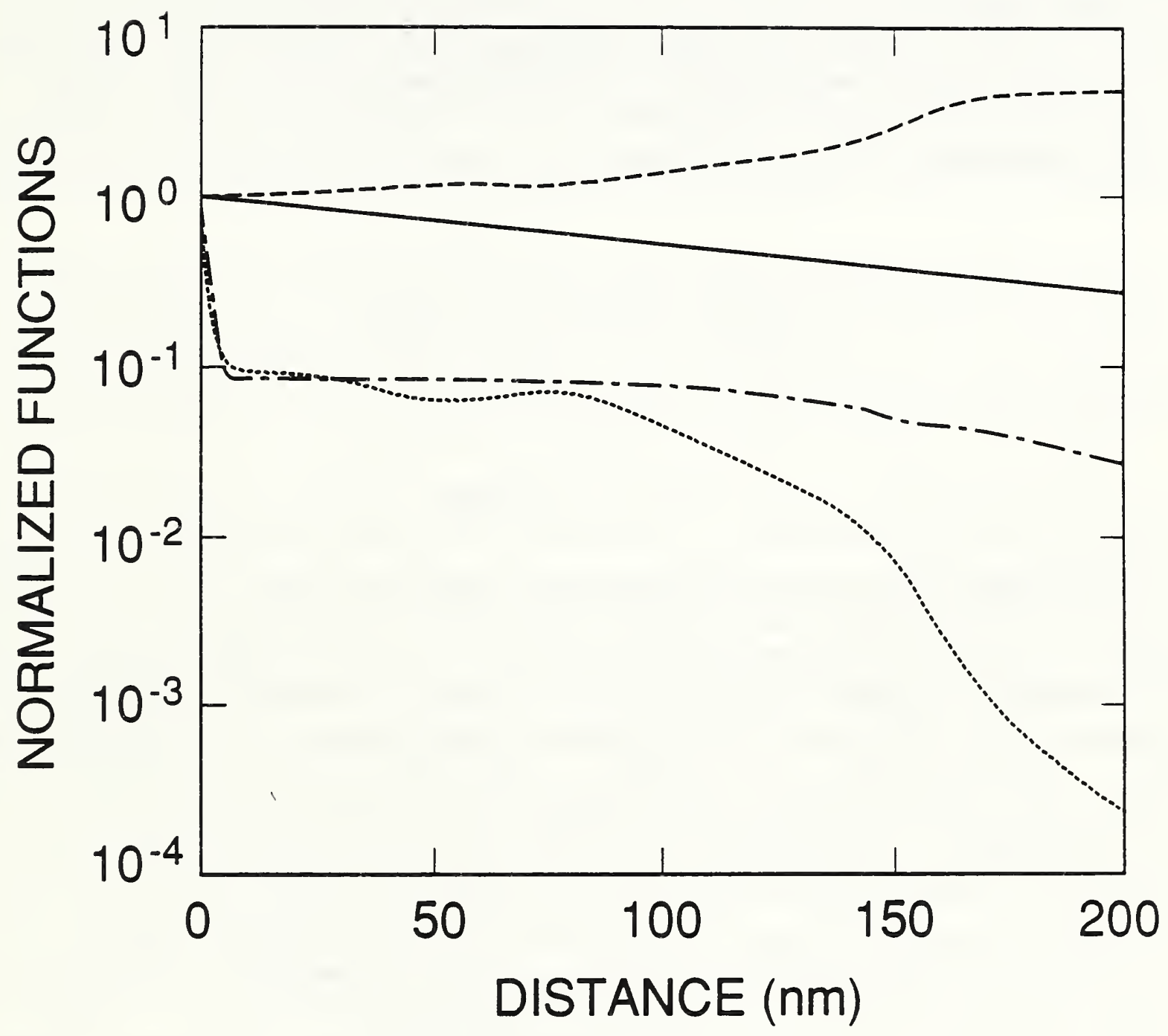

Figure 8. Comparison of $\hat{M}_{0}(x)$ (dotted line), $\hat{n}_{i e}(x)$ (dashed line), $\hat{\mu}_{m}(x)$ (dot-dashed line), and $\exp \left(-\alpha(400 \mathrm{~nm}) \mathrm{x}\right.$ ) (solid line) for a 1337 type photodiode with $N_{s s}, S$, and $\tau_{r}$ set as defined in Case 2 of table 1. The tildes in the preceding expressions indicate that these expressions have been normalized to unity at $x=0$, as shown for $k(x)$ in eq (10). 
of $\delta_{f}(\lambda)$ will become less dependent on the shape of $N_{A}(x) / N_{A}(0)$ because $\alpha(\lambda)$ decreases with increasing $\lambda$.

The numerical accuracy with which PC-1D calculates $M_{0}(x)$ within the framework of the drift-diffusion approximations for the conditions of charge accumulation at an oxide-silicon interface as shown in figure 8 has been studied previously and found to be quite good 7 . With a uniform doping density of $10^{19} \mathrm{~cm}^{-3}$ and an oxide charge density of $10^{13} \mathrm{~cm}^{-2}$, PC-1D overestimates $M_{0}(x)$ by about $9 \%$ at $x=0$, decreasing to within $\pm 1 \%$ for $x>1$ $\mathrm{nm}$. The uncertainty in $k(x)$ due to this source of error will be modeled by replacing $k(x)$ by

$$
k_{1}(x)=k(x) /\left(1-0.1 x / x_{0}\right)
$$

for $x<x_{0}$, and

$$
k_{1}(x)=k(x) / 0.9
$$

for $x>x_{0}$ in the kernel of the integral in eq (9), where $x_{0}=1 \mathrm{~nm}$.

Another source of error that must be considered is the numerical accuracy with which PC-1D would calculate the steady-state photocurrent if the equilibrium carrier concentration were exact. The uncertainty associated with this source of error was estimated as follows: 1) The internal quantum-deficiency spectrum was calculated directly from the PC-1D solution of the steady-state drift-diffusion equations for the total current flowing in the photodiode for the conditions described by Case 2 of table 1. 2) Equations (8)(13) were used to calculate the internal quantum-deficiency spectrum from the equilibrium values of $M_{0}(x), n_{i e}(x)$, and $\mu_{m}(x)$ calculated by PC-1D for the same conditions as in 1) above. The integration in eq (9) was carried out using a generalization of Simpson's rule for nonequidistant points. 3) Both spectra were normalized to 0.01 at $440 \mathrm{~nm}$, and the differences calculated. There is no reason to believe that these differences, which exceed $2 \times 10^{-5}$ only at $400 \mathrm{~nm}$ and at $900 \mathrm{~nm}$, are caused by errors associated with PC-1D. It is just as likely that they are associated with eqs (8-12) since these equations were derived from a number of assumptions that are not rigorously satisfied. The important point is that it is unlikely that either calculation is in error by much more than the differences between them. This verifies the numerical accuracy of the photocurrents calculated by PC-1D to the level of accuracy required in this paper.

There are a number of other errors, which are associated with incomplete or approximate physical models built into PC-1D, that also affect the accuracy with which PC-1D calculates the functions defining $k(x)$ in eq (13). These include quantum-mechanical effects (tunneling) [8] that change the shape of $M_{0}(x)$ from that calculated from the drift-diffusion approximation for $x<1 \mathrm{~nm}$, band-gap narrowing effects [9] associated with heavy doping and large surface-fields [10] that cause $n_{i e}(x)[6]$ to depend upon position in the photodiode, and carrier-carrier and carrier-ion scattering mechanisms that cause $\mu_{m}(x)$ to depend 
upon position 11. It is beyond the scope of this paper to investigate each of these sources of error in detail, but the uncertainty in $k(x)$ due to this source of error will be modeled by replacing $k(x)$ by

$$
k_{2}(x)=k(x)(1-0.1 \log (\hat{k}(x)))
$$

in the kernel of the integral in eq (9). This allows an error of $10 \%$ per decade change in $k(x)$.

The differences between the internal quantum-effiency spectrum calculated from eqs ( 8 13) by replacing $k(x)$ with either $k_{1}(x)$ or $k_{2}(x)$ in eq $(9)$ and the spectrum calculated using $k(x)$ were calculated after all three spectra were normalized to 0.01 at $440 \mathrm{~nm}$. Neither difference exceeded $2 \times 10^{-5}$ over the entire 400 to $900 \mathrm{~nm}$ spectral region. Therefore, all of these sources of error are considered negligible for the purposes of this paper.

Another source of error that is obvious in eqs (8-12) is the uncertainty associated with the absorption-coefficient data used in the simulations. As described in Part I, the absorptioncoefficient data were calculated from an equation [12] fitted to the data of Weakliem and Redfield [13]. An alternate set of absorption-coefficient data was described by Philipp [14. Figure 9 plots the differences between the internal quantum-deficiency spectra normalized to 0.01 at $440 \mathrm{~nm}$ for Case 2 of table 1 when based on the absorption-coefficient data in Ref. [14] and when based on the equation in Ref. [12]. These differences will be used as the estimated uncertainties arising from the uncertainties in the silicon absorption-coefficient spectrum when $\delta_{f}(\lambda)$ is used to extrapolate an internal quantum deficiency of 0.01 from $440 \mathrm{~nm}$ to longer wavelengths with a 1337 type photodiode. A similar calculation was carried out for the case where $S=0$ and $\tau_{r}=1 \mathrm{~ms}$. Both spectra were normalized to 0.002 at $860 \mathrm{~nm}$. The differences, which are shown in figure 10, are taken as the estimated uncertainties arising from the uncertainties in the silicon absorption-coefficient spectrum where $\delta_{r}(\lambda)$ is used to extrapolate an internal quantum deficiency of 0.002 from $860 \mathrm{~nm}$ to shorter wavelengths with a 1337 type photodiode.

Figures 11 and 12 plot the quadrature sum of the difference spectra plotted in figures 4 and 9 and in figures 7 and 10, respectively. The spectrum in figure 11 plots the estimated (one standard deviation) uncertainty as a function of wavelength that is associated with the use of $\delta_{f}(\lambda)$ in eq $(4)$ to extrapolate a measured internal quantum deficiency of 0.01 from $440 \mathrm{~nm}$ to longer wavelengths. The major sources of error contributing to the uncertainty spectrum in figure 11 are the diode-to-diode variations in $N_{A}(x) / N_{A}(0)$ and the uncertainty in the absorption-coefficient spectrum for silicon, but a number of other sources of error were identified with the help of eqs (8-12), and any that produced differences greater than $2 \times 10^{-5}$ for $400 \mathrm{~nm}<\lambda<900 \mathrm{~nm}$ are included in figure 11 .

The spectrum in figure 12 plots the estimated (one standard deviation) uncertainty as a function of wavelength that is associated with the use of $\delta_{r}(\lambda)$ in eq $(5)$ to extrapolate a measured internal quantum deficiency of 0.002 from $860 \mathrm{~nm}$ to shorter wavelengths. The major sources of error contributing to the uncertainty spectrum in figure 12 are the change 


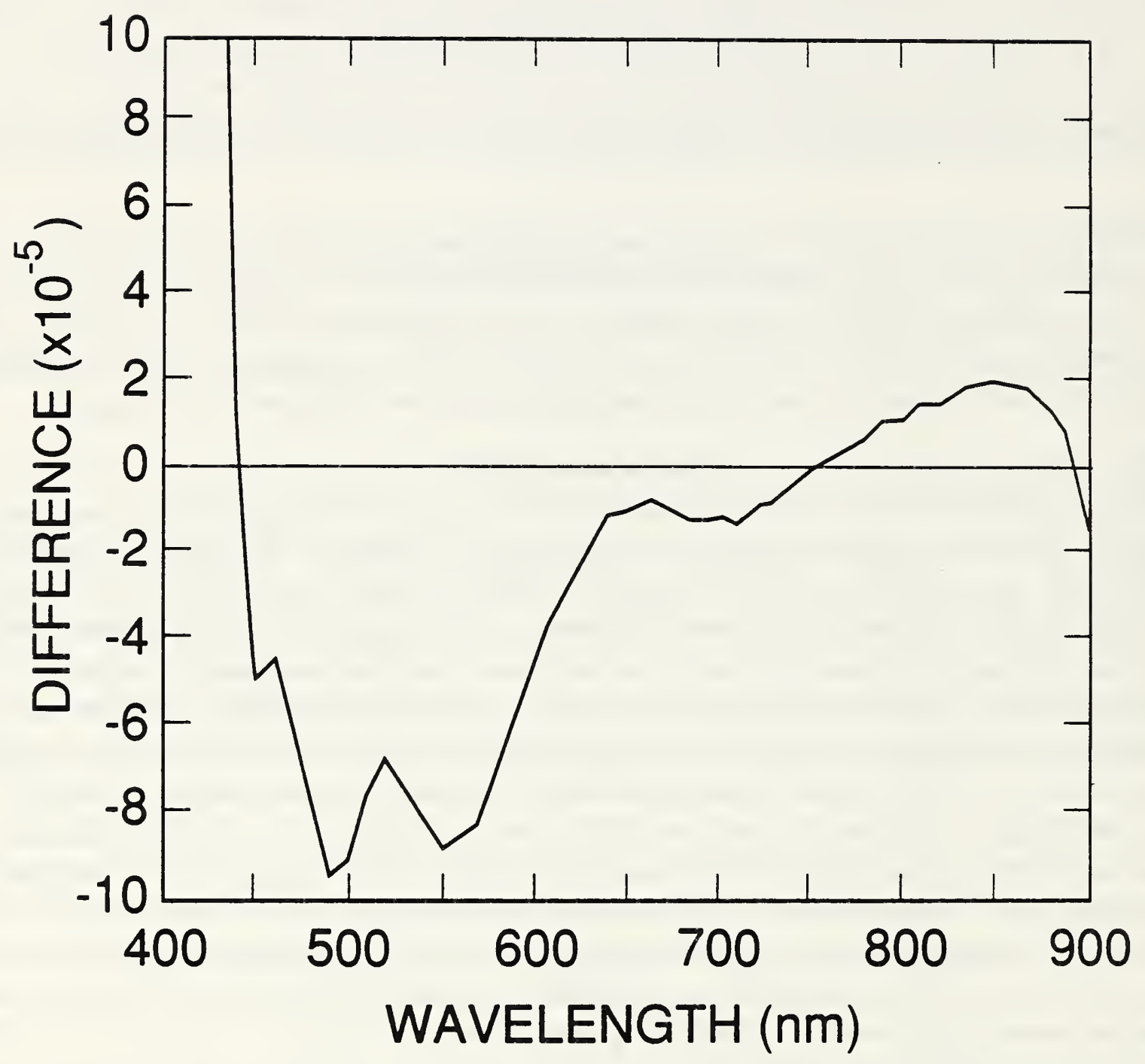

Figure 9. Differences between the internal quantum-deficiency spectrum for a 1337 type photodiode satisfying the conditions defined in table 1 for the absorption-coefficient data of Ref. [14] and for the absorption-coefficient data of Ref. [12]. 


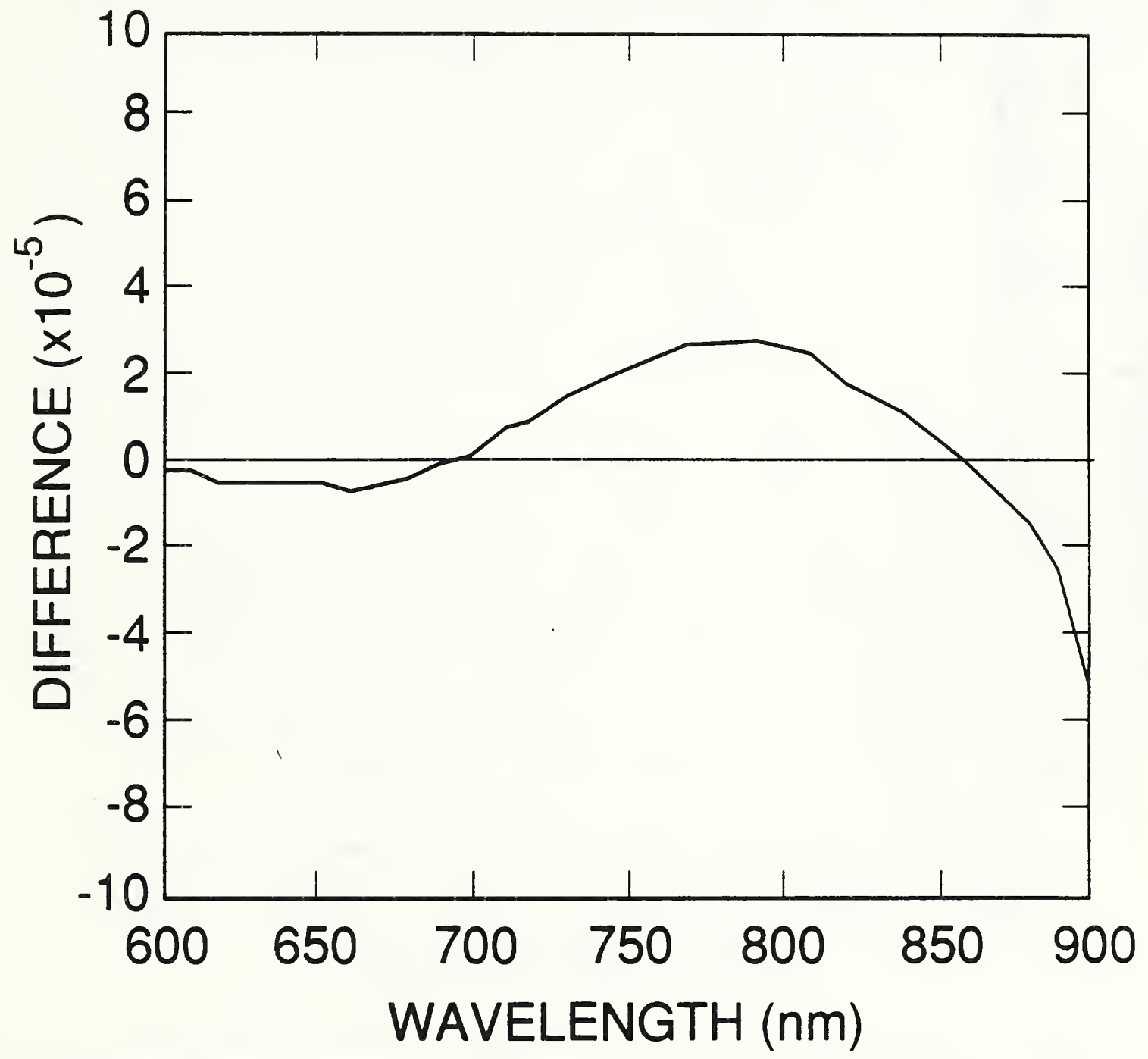

Figure 10. Differences between the internal quantum-deficiency spectra for a 1337 type photodiode with $S=0$ and $\tau_{r}=1 \mathrm{~ms}$ for the absorption-coefficient data of Ref. [14] and for the absorption-coefficient data of Ref. [12]. 


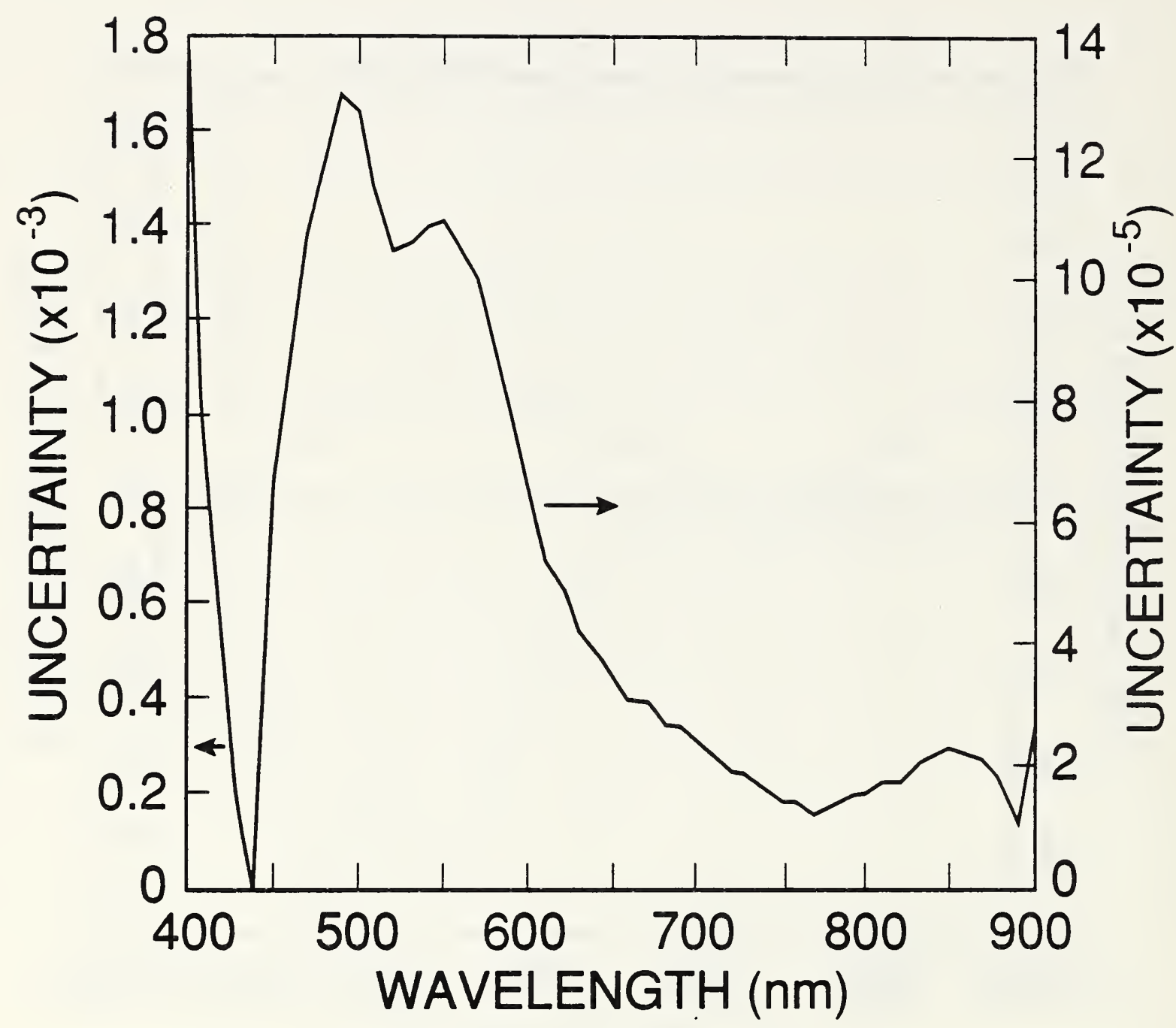

Figure 11. Sum in quadrature of the curves in figures 4 and 9 . This curve is the nominal uncertainty associated with the use of eq (4) in eq (2) to extrapolate a quantum deficiency of 0.01 at $440 \mathrm{~nm}$ to any other wavelength between 400 and $900 \mathrm{~nm}$. Notice that the sum is plotted on different scales below and above $440 \mathrm{~nm}$. 


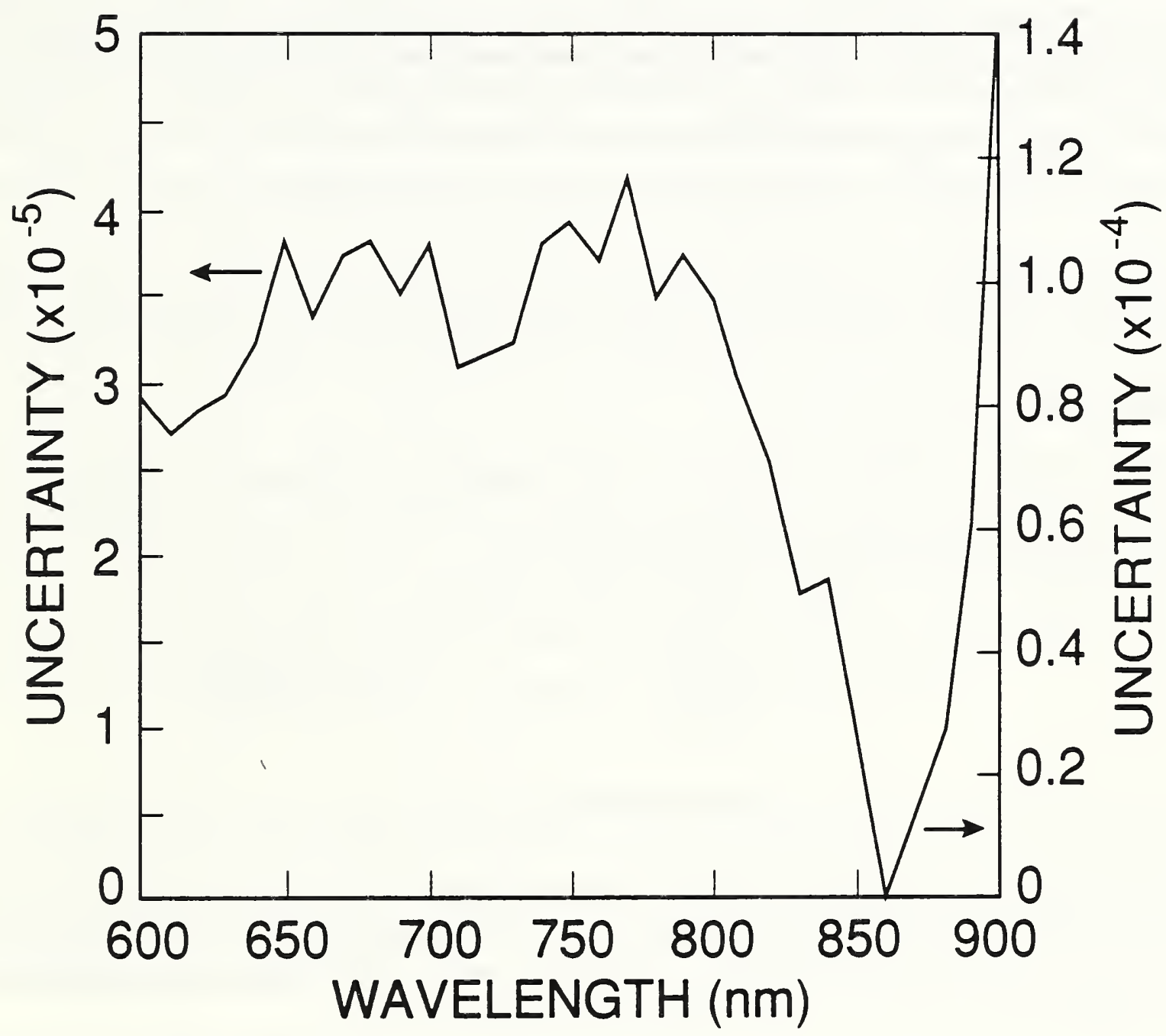

Figure 12. Sum in quadrature of the curves in figures 7 and 10. This curve is the nominal uncertainty associated with the use of eq (5) in eq (2) to extrapolate a quantum deficiency of 0.02 at $860 \mathrm{~nm}$ to any other wavelength between 400 and $900 \mathrm{~nm}$. Notice that the sum is plotted on different scales below and above $440 \mathrm{~nm}$. 
in shape of $\delta_{r}(\lambda)$ with $\tau_{r}$ and the uncertainty in the absorption-coefficient spectrum for silicon. All other sources of error considered were negligible with respect to $2 \times 10^{-5}$ for $400 \mathrm{~nm}<\lambda<900 \mathrm{~nm}$.

No equivalents to eqs (8-12) were used to guide the error analysis for $\delta_{r}(\lambda)$. The results of the uncertainty analysis of $\delta_{f}(\lambda)$ show that numerical accuracy of PC-1D will be a negligible source of error. This leaves the errors associated with the approximations in the physical models that are built into PC-1D. Some of these were tested, but no tests were possible for the use of a single SRH trap level to model the recombination in the rear region of the photodiode. This approximation could be a non-negligible source of error; it was shown in Part I that this approximation introduced errors of the order of the effect being modeled in nonlinearity simulations on a UV444B type photodiode. A more complete model might make $\tau_{r}$ depend upon position in the photodiode, which might modify the shape of $\delta_{r}(\lambda)$ and add an uncertainty that is not included in figure 12.

\section{Comparison with Experiment}

To use the results developed thus far, it is necessary to define the internal quantumdeficiency interpolating function $\delta(\lambda)$, and to assume that it exactly satisfies eqs (1-3), which can be rewritten in more compact form as

$$
\delta(\lambda)=\delta_{z}\left(\lambda_{f}\right) \frac{\delta_{f}(\lambda)}{\delta_{f}\left(\lambda_{f}\right)}+\delta_{z}\left(\lambda_{r}\right)\left[1-\frac{\delta_{f}\left(\lambda_{r}\right)}{\delta_{r}\left(\lambda_{r}\right)}\right] \frac{\delta_{r}(\lambda)}{\delta_{r}\left(\lambda_{r}\right)}
$$

where $\delta_{z}\left(\lambda_{f}\right)$ and $\delta_{z}\left(\lambda_{r}\right)$ are the measured values of the internal quantum deficiency at the wavelengths $\lambda_{f}$ and $\lambda_{r}$, respectively; $\delta_{f}(\lambda)$ and $\delta_{r}(\lambda)$ are defined in eqs (4) and (5), respectively, and the constants used in these equations are defined in table 2 . The uncertainty associated with $\delta(\lambda)$ due to the sources of error considered in the last two sections of this paper can be obtained by summing in quadrature each of the terms on the right-hand side of the differential of eq (17). That differential is given by

$$
\begin{aligned}
d \delta(\lambda) & =d \delta_{z}\left(\lambda_{f}\right)\left[\frac{\delta_{f}(\lambda)}{\delta_{f}\left(\lambda_{f}\right)}\right] \\
& +d \delta_{\boldsymbol{z}}\left(\lambda_{r}\right)\left[\frac{\delta_{r}(\lambda)}{\delta_{r}\left(\lambda_{r}\right)}\right]\left[1-\frac{\delta_{f}\left(\lambda_{r}\right)}{\delta_{r}\left(\lambda_{r}\right)}\right] \\
& +d \delta_{f}(\lambda)\left[\frac{\delta_{z}\left(\lambda_{f}\right)}{\delta_{f}\left(\lambda_{f}\right)}\right] \\
& +d \delta_{r}(\lambda)\left[\frac{\delta_{x}\left(\lambda_{r}\right)}{\delta_{r}\left(\lambda_{r}\right)}\right]\left[1-\frac{\delta_{f}\left(\lambda_{r}\right)}{\delta_{r}\left(\lambda_{r}\right)}\right] .
\end{aligned}
$$


The differentials $d \delta_{f}(\lambda)$ and $d \delta_{r}(\lambda)$ are plotted in figures 11 and 12 for $\lambda_{f}=440 \mathrm{~nm}$, and for $\lambda_{r}=860 \mathrm{~nm}$, respectively.

There are not many data available against which to test eqs (17) and (18), but there are some. For instance, Zalewski and Hoyt [4] have reported the internal quantum efficiencies at $441.6 \mathrm{~nm}$ and the spectral responsivities and absorptances at $633.0 \mathrm{~nm}$ for five multiple reflection (trap) [4,15-17] radiometers based on Hamamatsu 1337 photodiodes at 441.6 and $633.0 \mathrm{~nm}$. Similarly, Fox [17] has reported the average internal quantum efficiency of ten trap radiometers also based on Hamamatsu 1337 photodiodes at six $A r^{+}$ion laser lines, and he has also measured the oxide-bias correction for a single Hamamatsu 1337 photodiode at four $\mathrm{Ar}^{+}$ion laser lines.

Zalewski and Hoyt [4] presented their data, which are summarized in bold face type in table 3 , as an intercomparison of spectral responsivity. Since the internal quantum deficiency is the quantity of interest in this paper, it is necessary to compute this quantity from the data reported by $Z$ alewski and Hoyt as shown in table 3. The corrections for nonlinearity that $\mathrm{Zalewski}$ and Hoyt derived from reverse-bias measurements also eliminate any effects of recombination in the rear region of the photodiodes.

Fox's [17] oxide-bias data, which are summarized in table 4, were measured at $10 \mathrm{~V}$, and were reported as a fractional increase in photocurrent $I$ given by $\gamma_{0}=I(10 \mathrm{~V}) / I(0)-1$. The oxide-bias experiment reported in Part II shows that the internal quantum efficiency at zero bias $\delta_{z}$ is given by

$$
\delta_{x}=1-1 /\left(1+1.13 \gamma_{0}\right)
$$

where the maximum oxide bias-voltage is $10 \mathrm{~V}$. The average internal quantum efficiencies reported by Fox are also shown in table 4, and the internal quantum deficiencies calculated from them are also shown there.

Equation (17) was normalized to the results of Zalewski and Hoyt at $\lambda_{f}=441.6 \mathrm{~nm}$, and the predictions of that equation are compared with the results of Zalewski and Hoyt at 633 $\mathrm{nm}$ in table 5. Note that $\delta_{\boldsymbol{x}}\left(\lambda_{r}\right)=0$ for this data set due to the application of reverse bias. Equation (17) was also normalized to the oxide-bias results of Fox at $\lambda_{f}=468.18 \mathrm{~nm}$, and the predictions of that equation are compared with the oxide-bias results in table 6 . Again note that $\delta_{x}\left(\lambda_{r}\right)=0$ because oxide-bias measurements are not sensitive to recombination in the rear of the photodiode.

The uncertainty estimates for eq (17) in tables 5 and 6 made use of the data in figure 11 even though those data apply to $\lambda_{f}=440 \mathrm{~nm}$ rather than 441.6 or $468.2 \mathrm{~nm}$. This does not distort the estimated uncertainty significantly because the terms are added in quadrature, and the first term on the right-hand side of eq (18) is larger than the second term for both sets of data. Fox's data point at $406.85 \mathrm{~nm}$ was included in table 6 even though the uncertainties associated with extrapolating $\delta_{f}(\lambda)$ to wavelengths shorter than $\lambda_{f}$ are very unfavorable for high-accuracy applications. The good agreement at this wavelength must 
Table 3. Average values of, and uncertainty estimates for the internal quantum efficiency $\epsilon_{f}$, spectral responsivity $\mathrm{R}$, one minus the reflectance $(1-\rho)$ and nonlinearity correction (1-NL) reported in Ref. [5] (bold face type) at wavelength $\lambda$ for five multiple-reflection radiometers based on 1337 type photodiodes, and internal quantum-deficiency values $\delta_{\boldsymbol{x}}(\lambda)$ calculated from the reported data.

\begin{tabular}{|c|c|c|c|c|c|}
\hline $\begin{array}{c}\lambda \\
(\mathrm{nm})\end{array}$ & $\epsilon_{f}$ & $\begin{array}{c}R \\
(A / W)\end{array}$ & $(1-\rho)$ & $(1-\mathrm{NL})$ & $\delta_{z}(\lambda)$ \\
\hline 441.6 & $\begin{array}{l}+0.99347 \\
\pm 0.00040\end{array}$ & & & & $\begin{array}{l}+0.00653 \\
\pm 0.00040\end{array}$ \\
\hline 633.0 & +0.50840 & $\begin{array}{l}+0.99704 \\
\pm 0.00009\end{array}$ & $\begin{array}{l}+0.99972 \\
\pm 0.00014\end{array}$ & $\begin{array}{r}+0.00097 \\
\pm 0.00004\end{array}$ & \pm 0.00023 \\
\hline
\end{tabular}

Table 4. Oxide-bias data $\gamma_{o}$ reported in Ref. [17] for a single 1337 type photodiode, the average internal quantum-efficiency data $\epsilon_{x}$ reported in Ref. [17] for ten multiple-reflection radiometers based on 1337 type photodiodes, and the quantum deficiencies $\delta_{x}(\lambda)$ calculated from these sets of data.

\begin{tabular}{|c|c|c|c|c|}
\hline \multirow{2}{*}{$\begin{array}{c}\lambda \\
(\mathrm{nm})\end{array}$} & \multicolumn{2}{|c|}{ - oxide bias - } & \multicolumn{2}{|c|}{ - iqe - } \\
\hline & $\gamma_{0}$ & $\delta_{x}(\lambda)$ & $\epsilon_{2}$ & $\delta_{\boldsymbol{x}}(\lambda)$ \\
\hline 406.85 & $\begin{array}{l}+0.0126 ? \\
\pm 0.00015\end{array}$ & $\begin{array}{l}+0.01404 \\
\pm 0.00017\end{array}$ & $\begin{array}{l}+0.9867 \\
\pm 0.0002\end{array}$ & $\begin{array}{l}+0.0133 \\
\pm 0.0002\end{array}$ \\
\hline 468.18 & $\begin{array}{l}+0.0044 ? \\
\pm 0.00015\end{array}$ & $\begin{array}{l}+0.00495 \\
\pm 0.00017\end{array}$ & $\begin{array}{l}+0.9934 \\
\pm 0.0003\end{array}$ & $\begin{array}{l}+0.0066 \\
\pm 0.0003\end{array}$ \\
\hline 568.35 & & & $\begin{array}{c}+0.9977 \\
\pm 0.00020\end{array}$ & $\begin{array}{l}+0.0023 \\
\pm 0.0002\end{array}$ \\
\hline 647.30 & $\begin{array}{l}+0.0007 ? \\
\pm 0.00015\end{array}$ & $\begin{array}{l}+0.00079 \\
\pm 0.00017\end{array}$ & $\begin{array}{l}+0.9986 \\
\pm 0.0002\end{array}$ & $\begin{array}{l}+0.0014 \\
\pm 0.0002\end{array}$ \\
\hline 799.54 & & & $\begin{array}{l}+0.9989 \\
\pm 0.0002\end{array}$ & $\begin{array}{l}+0.0011 \\
\pm 0.0002\end{array}$ \\
\hline 859.07 & $\begin{array}{l}+0.0000 ? \\
\pm 0.00015\end{array}$ & $\begin{array}{l}+0.00000 \\
\pm 0.00017\end{array}$ & $\begin{array}{l}+0.9987 \\
\pm 0.0002\end{array}$ & $\begin{array}{l}+0.0013 \\
\pm 0.0002\end{array}$ \\
\hline
\end{tabular}


Table 5. Comparison of predictions of eq (17) with measured internal quantum-deficiency data $\delta_{x}(\lambda)$ in table 3 for $\lambda_{f}=441.6 \mathrm{~nm}$ and $\delta_{x}\left(\lambda_{r}\right)=0$.

$\begin{array}{cccc}\begin{array}{c}\text { Prediction } \\ \text { Wavelength }\end{array} & \begin{array}{c}\text { Measured } \\ \text { Value } \\ (\mathrm{nm})\end{array} & \begin{array}{c}\text { Predicted } \\ \text { Value } \\ \text { Ref. }[4]\end{array} & \text { Difference } \\ & & & \text { eq (17) } \\ 633.0 & +0.00097 & +0.00089 & -0.00008 \\ & \pm 0.00023 & \pm 0.00007 & \pm 0.00024\end{array}$

Table 6. Comparison of predictions of eq (17) with internal quantum-deficiency data $\delta_{x}(\lambda)$ derived from oxide-bias data in table 4 for $\lambda_{f}=468.18 \mathrm{~nm}$ and $\delta_{x}\left(\lambda_{r}\right)=0$.

$\begin{array}{cccc}\begin{array}{c}\text { Prediction } \\ \begin{array}{c}\text { Wavelength } \\ \text { (nm) }\end{array}\end{array} & \begin{array}{c}\text { Measured } \\ \text { Value } \\ \text { Ref. [15] }\end{array} & \begin{array}{c}\text { Predicted } \\ \text { Value } \\ \text { eq }(17)\end{array} & \text { Difference } \\ 406.85 & & & \\ & +0.01404 & +0.01321 & -0.00083 \\ & \pm 0.00017 & \pm 0.01144 & \pm 0.01144 \\ 647.30 & & & \\ & +0.00079 & +0.00090 & +0.00010 \\ 859.07 & \pm 0.00017 & \pm 0.00030 & \pm 0.00034 \\ & & & \\ & +0.00000 & +0.00015 & +0.00015 \\ & \pm 0.00017 & \pm 0.00005 & \pm 0.00018\end{array}$


be considered fortuitous considering the uncertainty associated with the predicted value. The differences at the other wavelengths never exceeds 0.00015 , and they fall within the estimated uncertainty for the differences.

Table 7 and figure 13 compare the internal quantum-deficiency results of Fox [17] with the predictions of eq (17) when normalized at $\lambda_{f}=468.18 \mathrm{~nm}$ and at $\lambda_{r}=859.07 \mathrm{~nm}$. The data in figures 11 and 12 that apply for $\lambda_{f}=440 \mathrm{~nm}$ and for $\lambda_{r}=860 \mathrm{~nm}$, respectively, are used with eq (18) to estimate the uncertainties assigned to the predictions of eq (17). The difference at $406.85 \mathrm{~nm}$ is much larger than that obtained with the oxide-bias data but still falls within the estimated uncertainty. Because the oxide-bias experiment is not sensitive to the quantum yield for electron-hole pair production, it is tempting to imagine that the discrepancy between the oxide bias and internal quantum efficiency reflects the fact that the quantum yield for the 1337 type photodiode is greater than unity at $406 \mathrm{~nm}$. However, the fact that this discrepancy is not statistically significant at the one-sigma level shows that this conclusion cannot be drawn from the data presented here. The differences at the other wavelengths never exceed 0.0003 , and only fall outside the estimated uncertainties for the differences at $799.54 \mathrm{~nm}$.

The results shown in tables 5 through 7 and figure 13 verify that the internal quantum deficiency spectrum of multiple-reflection radiometers based on Hamamatsu 1337 photodiodes can be interpolated over the 440 to $860 \mathrm{~nm}$ spectral region from only two measured quantum deficiencies, one at each end of the region. This greatly reduces the number of measurements needed for high-accuracy spectral calibrations of these photodiodes and radiometers. Furthermore, the theoretical uncertainty analysis suggests that the uncertainties in the interpolated values will be less than or equal to the uncertainties in the measured values, at least as long as the latter are greater than \pm 0.0001 . The results in tables 5 to 7 and figure 13 do not contradict this idea, but the experimental data are not accurate enough to confirm it. On the other hand, the experimental data do confirm that an uncertainty of \pm 0.0003 is obtainable.

\section{Conclusion}

Part III has shown that Version 2 of the semiconductor-device modeling program PC-1D can be used to model the spectral shape of the internal quantum deficiency of 1337 type photodiodes with very small uncertainty over the $440 \mathrm{~nm}$ spectral region. The largest uncertainties are caused by uncertainties in the absorption-coefficient spectrum of silicon, diode-to-diode variations in the shape of the front region dopant distribution, and diodeto-diode variations in the position-independent rear-region minority-carrier lifetime. It was not possible to estimate the uncertainty caused by approximating the rear-region minority-carrier lifetime as being independent of position.

Simple formulae were derived that allow high-accuracy internal quantum-efficiency calibrations on 1337 type photodiodes to be interpolated from measured values at the ends of the 440 to $860 \mathrm{~nm}$ spectral region over the interior of that region. The uncertainties that can be obtained with these formulae are comparable to those that can be obtained with the highest accuracy measurements currently available. 
Table 7. Comparison of predictions of eq (17) with measured internal quantum-deficiency data in table 4 for $\lambda_{f}=468.18 \mathrm{~nm}$ and $\lambda_{r}=859.07 \mathrm{~nm}$.

$\begin{array}{cccc}\begin{array}{c}\text { Prediction } \\ \begin{array}{c}\text { Wavelength } \\ \text { (nm) }\end{array}\end{array} & \begin{array}{c}\text { Measured } \\ \text { Value } \\ \text { Ref. }[15]\end{array} & \begin{array}{c}\text { Predicted } \\ \text { Value } \\ \text { eq }(17)\end{array} & \text { Difference } \\ 406.85 \mathrm{~nm} & +0.0133 ? & +0.01744 & \\ & \pm 0.00025 & \pm 0.01000 & \pm 0.00414 \\ & +0.0023 ? & +0.00224 & -0.00006 \\ 568.35 \mathrm{~nm} & \pm 0.00030 & \pm 0.00007 & \pm 0.00031 \\ & & & \\ 647.30 \mathrm{~nm} & +0.0014 ? & +0.00121 & -0.00019 \\ & \pm 0.00020 & \pm 0.00004 & \pm 0.00020 \\ 799.54 \mathrm{~nm} & +0.0011 ? & +0.00082 & -0.00028 \\ & \pm 0.00021 & \pm 0.00009 & \pm 0.00023\end{array}$




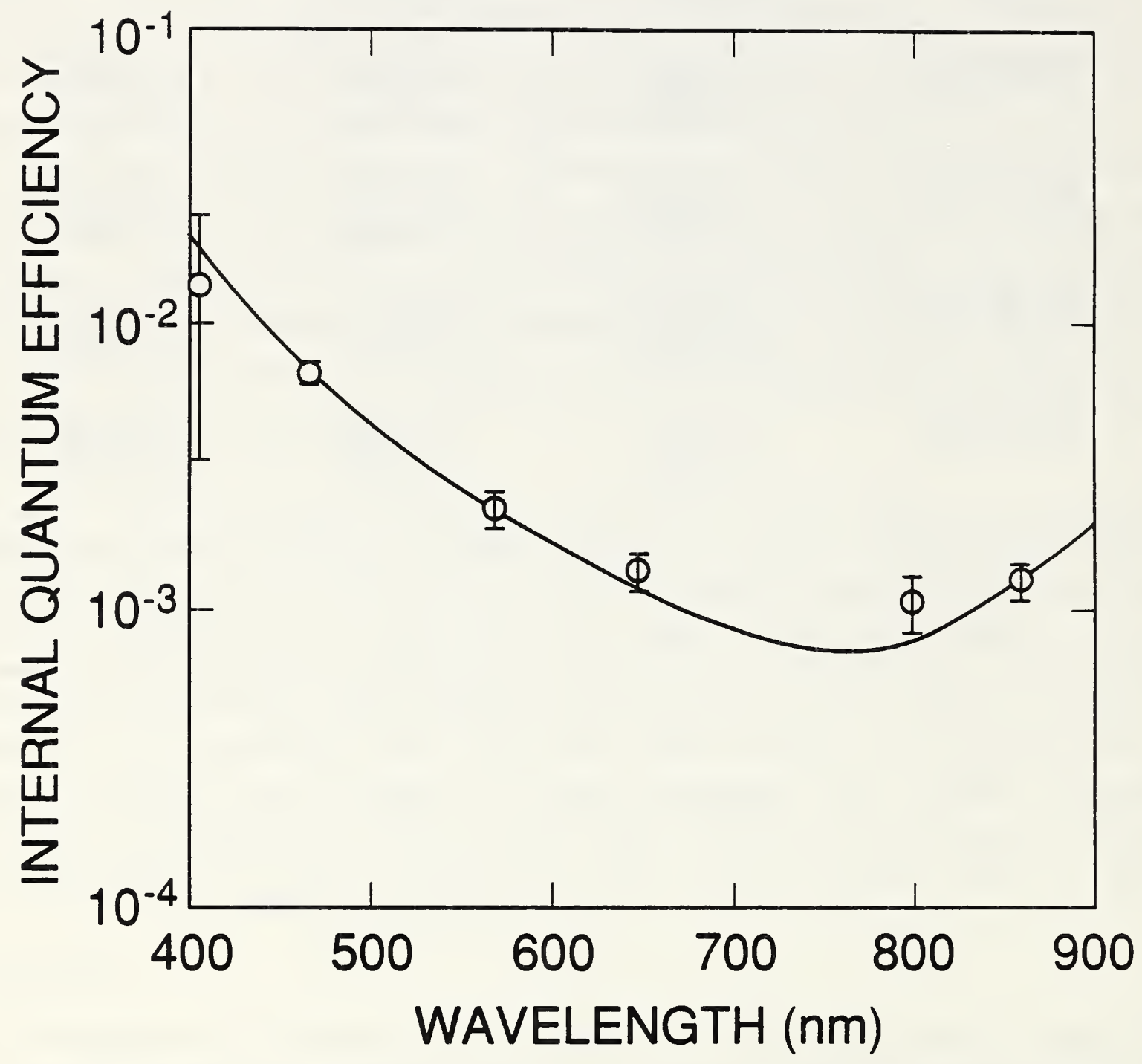

Figure 13. Comparison of measured data of Ref. [17] (open circles) with eq (17) (solid line) normalized to pass through the measured point at 468.18 and $859.1 \mathrm{~nm}$. At these wavelengths the error bars are the uncertainties in the measured values; at the other wavelengths the error bars are the quadrature sum of the uncertainties in the measured values and those associated with the use of eq (17). 
5. References

[1] Geist, J., Appl. Opt. 18, 760 (1979).

[2] Geist, J., Zalewski, E. F., and Schaefer, A. Appl. Opt. 19, 3795 (1980).

[3] Hoyt, C. C., Miller, P. J., Foukal, P. V., and Zalewski, E. F., SPIE 1109, 236 (1989).

[4] Zalewski, E. F., and Hoyt, C. C., Metrologia 28, 203 (1991).

[5] Geist, J., and Baltes, H., Appl. Opt. 28, 3929 (1989).

[6] Slotboom, J. W., and de Graaff, H. C., Solid-State Electron. 19, 857 (1976).

[7] Geist, J., Lowney, J. R., James, C. R., and Robinson, A. M., J. Appl. Phys. 70, 236 (1991).

[8] Ohkura, Y., Solid-State Electron. 33, 1581 (1990).

[9] Bennett, H. S., and Lowney, J. R., Solid-State Electron. 33, 675 (1990).

[10] Girisch, R. B. M., Mertens, R. P., and Verbeke, O. B., Solid-State Electron. 33, 85 (1990).

[11] Bennett, H. S., Solid-State Electron. 26, 1157 (1983).

[12] Geist, J., Migdall, A., and Baltes, H. P., Appl. Opt. 27, 3777 (1988).

[13] Weakliem, H. A., and Redfield, D., J. Appl. Phys. 50, 1491 (1979), and personal communication.

[14] Philipp, H. R., J. Appl. Phys. 43, 2835 (1972), and personal communication.

[15] Zalewski, E. F., and Duda, C., Appl. Opt. 22, 2867 (1983).

[16] Fox, N. P., and Martin, J. E., Appl. Opt. 29, 4686 (1990).

[17] Fox, N. P., Metrologia 28, 197 (1991), and personal communication. 



\begin{tabular}{|c|c|c|}
\hline \multirow[t]{3}{*}{$\begin{array}{l}\text { NIST-114A } \\
\text { (REV. 3-90) }\end{array}$} & \multirow{3}{*}{$\begin{array}{l}\text { U.S. DEPARTMENT OF COMMERCE } \\
\text { NATIONAL INSTITUTE OF STANDARDS AND TECHNOLOGY } \\
\text { BIBLIOGRAPHIC DATA SHEET }\end{array}$} & $\begin{array}{l}\text { 1. PUBUCATION OR REPORT NUMBER } \\
\text { NISTIR } 4592\end{array}$ \\
\hline & & 2. PERFORMING OROANIZATION REPORT NUMBER \\
\hline & & $\begin{array}{l}\text { 3. PUBUCATION DATE } \\
\text { January } 1992\end{array}$ \\
\hline
\end{tabular}

4. TITLE AND SUBTITLE

Numerical Modeling of Silicon Photodiodes for High-Accuracy Applications

5. AUTHOR(S)

J. Geist, D. Chandler-Horowitz, A. M. Robinson, C. R. James, R. Kohler, and R. Goebel

6. PERFORMINQ ORGANIZATION (IF JOINT OR OTHER THAN NIST, SEE INSTRUCTIONS)

U.S. DEPARTMENT OF COMMERCE

NATIONAL INSTITUTE OF STANDARDS AND TECHNOLOQY

GAITHERSBURG, MD 20890

7. CONTRACT/ORANT NUMBER

8. TYPE OF REPORT AND PERIOD COVEAED

9. SPONSORING ORGANIZATION MAME AND COMPLETE ADDRESS (STREET, CITY, STATE, ZIP)

10. SUPPLEMENTARY NOTES

11. ABSTRACT (A 200-WORD ON LESS FACTUAL SUMMARY OF MOST SIGMIFICANT INFOAMATION. IF DOCUMENT INCLUDES A SIGNIFICANT BIBUOGRAPHY OR UTERATURE SUAVEY, MENTION IT HERE.)

The purpose of this National Institute of Standards and Technology Interagency/Internal Report (NISTIR) is to provide the source code for two Turbo Pascal 5.5 programs and an MSDOS batch program, along with a paper that describes the programs and provides examples of their use. These three programs serve as a batch mode interface to support highaccuracy photodiode modeling with Version 2 of the semiconductor device modeling program PC-1D. These programs are useful because the interactive user interface of PC-lD is optimized for solar cell modeling, and it is somewhat difficult to access the highest accuracy available from PC-1D through this interface. Part I describes PC-lD from the point of view of high-accuracy photodiode modeling and describes the programs that support its use in this application. Parts II and III present examples of the use of the programs described in Part I to model two different types of experiments performed on silicon photodiodes in various high-accuracy applications.

12. KEY WORDS (6 TO 12 ENTAIES; ALPHABETICAL ORDER; CAPTTMZE ONLY PROPER MAMES; AND SEPARATE KEY WORDS OY SEMICOLONS) numerical modeling; numerical simulation; oxide-bias experiment; photodiode modeling; quantum efficiency; silicon; silicon photodiodes

\section{AVALABIUTY}

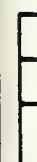

UNUMITED

FOR OFFICLAL DISTRIEUTION. DO NOT RELEASE TO MATIOMAL TECHMICAL INFONMATION SERVICE (NTIS).

ORDER FROM SUPERINTENDENT OF DOCUMENTS, U.S. GOVERMMENT PAINTING OFFICE, WASHINOTON, DC 20402

ORDER FROM MATIONAL TECHMICAL INFORMATION SERVICE (NTIS), SPRINGFIEL, VA 20161.

14. NUMBER OF PRINTED PAGES

92

15. PRICE

A0 5 

.

the $\therefore$ 
